\title{
ON $d$-DIMENSIONAL COMPACT HYPERBOLIC COXETER POLYTOPES WITH $d+4$ FACETS
}

\author{
PAVEL TUMARKIN AND ANNA FELIKSON
}

\begin{abstract}
We prove that there are no compact Coxeter polytopes with $d+4$ facets in a hyperbolic space of dimension $d>7$. This estimate is sharp: examples of such polytopes in dimensions $d \leq 7$ were found by V. O. Bugaenko in 1984. We also show that in dimension 7 there is a unique polytope with 11 facets.
\end{abstract}

\section{INTRODUCTION}

A polytope in a hyperbolic space is called a Coxeter polytope if all its dihedral angles are of the form $\frac{\pi}{k_{i j}}$ for some integers $k_{i j} \geq 2$. Any Coxeter polytope is a fundamental domain of the discrete group generated by the reflections in its facets. A complete classifications of compact hyperbolic Coxeter polytopes is not yet known. Vinberg [18] showed that there are no such polytopes in dimensions $d \geq 30$. The known examples are only in dimensions $d \leq 8$ (see [5] and [6]). In dimensions 2 and 3, compact hyperbolic Coxeter polytopes were completely classified by H. Poincaré 15 and Andreev [3. Compact hyperbolic Coxeter polytopes of the simplest combinatorial type, i.e., simplices were classified by Lannér [14. Kaplinskaya [13. (see also [19]) classified all simplicial prisms, Esselmann [9] classified the remaining $d$-dimensional compact hyperbolic Coxeter polytopes with $d+2$ facets. In [8], Esselmann proved that $d$-dimensional compact Coxeter polytopes with $d+3$ facets exist only in dimensions 8 and lower, and also showed that in dimension 8 there is only one such polytope. Compact $d$-dimensional Coxeter polytopes with $d+3$ facets in dimensions 4 through 7 were classified by P. Tumarkin [17].

In this paper we investigate the next-in-complexity class of polytopes, namely, $d$ dimensional compact hyperbolic Coxeter polytopes with $d+4$ facets. We prove that there are no such polytopes in a hyperbolic space of dimension $d \geq 8$. In dimensions $2 \leq d \leq 7$ such polytopes do exist [5]. We also prove that in dimension $d=7$ there is only one such polytope.

The paper is organized as follows. Section 1 is of auxiliary nature: we recall basic results about Coxeter diagrams and the combinatorics of simple polytopes. We also mention some facts connecting combinatorial (metric) properties of the faces of a polytope and combinatorial (metric) properties of the polytope itself. Section 2 is devoted to Coxeter diagrams that do not contain Lannér subdiagrams of order less than 5 . In particular, we show that the Coxeter diagram of any compact hyperbolic Coxeter polytope contains a Lannér subdiagram of order less than 5. In Section 3 we develop a theory of liftings, which connects the combinatorics of a face of a Coxeter polytope and a certain

2000 Mathematics Subject Classification. Primary 52B11; Secondary 20 F55.

The first author was partially supported by the President of the Russian Federation grants MK6290.2006.1 and NSh-5666.2006.1, the RFFI grant No. 07-01-00390-a, and the INTAS grant YSF-0610000014-5766.

The second author was partially supported by the President of the Russian Federation grant NSh5666.2006.1, the RFFI grant No. 07-01-00390-a, and the INTAS grant YSF-06-10000014-5916. 
subdiagram of the Coxeter diagram of this polytope. In Sections 4 and 5 we apply the obtained results to prove the non-existence of the above polytopes in dimensions $d \geq 8$. Finally, in Section 6 we prove that in a hyperbolic space of dimension 7 there is only one compact Coxeter polytope with 11 facets.

This work was partially done at the Max Planck Institute in Bonn. The authors thank the Institute for hospitality.

\section{Preliminaries}

In this section we collect basic facts about Coxeter diagrams, Gale diagrams, and diagrams of missing faces. In our discussion of Coxeter diagrams we mainly follow [19] and [20. For details about Gale diagrams, see [12] and [7] (see also [8, where connections between Coxeter polytopes, Gale diagrams, and diagrams of missing faces are explained). At the end of the section, we mention a recent result of D. Allcock [1] showing that most Coxeter polytopes have a Coxeter face, and explaining how to construct the Coxeter diagram of a Coxeter face from the Coxeter diagram of the polytope.

1.1. Coxeter diagrams. 1. An abstract Coxeter diagram $\Sigma$ is a finite one-dimensional simplicial complex, whose edges are assigned positive weights $w_{i j}$, where $w_{i j}=\cos \frac{\pi}{m_{i j}}$ for some integer $m_{i j} \geq 3$ whenever $w_{i j}<1$. A subdiagram of $\Sigma$ is a subcomplex whose edges are labeled by the same weights as in $\Sigma$. The order $|\Sigma|$ of the diagram $\Sigma$ is the number of its nodes.

The union of subdiagrams $\Sigma_{1}$ and $\Sigma_{2}$ of $\Sigma$ is the subdiagram $\left\langle\Sigma_{1}, \Sigma_{2}\right\rangle$ spanned by the nodes of $\Sigma_{1}$ and $\Sigma_{2}$. By definition, the subdiagrams $\Sigma_{1} \backslash v$ and $\Sigma_{1} \backslash \Sigma_{2}$ of $\Sigma_{1}$ are spanned by the nodes of $\Sigma_{1}$ without $v$, respectively, without the nodes of $\Sigma_{2}$.

Given an abstract Coxeter diagram $\Sigma$ with nodes $v_{1}, \ldots, v_{n}$ and weights $w_{i j}$ we construct a symmetric $(n \times n)$-matrix $G(\Sigma)=\left(g_{i j}\right)$ such that $g_{i i}=1$, and for $i \neq j$, $g_{i j}=-w_{i j}$ if $v_{i}$ and $v_{j}$ are joined, and $g_{i j}=0$ otherwise. We define the determinant $\operatorname{det}(\Sigma)$ and the signature of $\Sigma$ as the determinant and, respectively, the signature of $G(\Sigma)$.

We draw the edges of a Coxeter diagram in the following way: if the weight equals $\cos \frac{\pi}{m_{i j}}$, then the corresponding nodes are joined by an $\left(m_{i j}-2\right)$-fold edge or a simple edge labeled by $m_{i j}$; if the weight equals 1 , the nodes are joined by a bold edge; if the weight is greater than 1 , then the nodes are joined by a dashed edge labeled by the weight (or without a label).

We write $\left[v_{i}, v_{j}\right]=m_{i j}$ if $w_{i j}=\cos \frac{\pi}{m_{i j}}$, and $\left[v_{i}, v_{j}\right]=\infty$ if $v_{i} v_{j}$ is a dashed edge. The notation $\left[v_{i}, v_{j}\right]=2$ indicates that $v_{i}$ and $v_{j}$ are not joined.

An abstract Coxeter diagram $\Sigma$ is said to be elliptic if $G(\Sigma)$ is positive definite; $\Sigma$ is parabolic if each indecomposable component of $G(\Sigma)$ is degenerate and positive semidefinite; a connected diagram $\Sigma$ is said to be Lannér if $\Sigma$ is neither elliptic nor parabolic but any proper subdiagram of $\Sigma$ is elliptic; $\Sigma$ is said to be hyperbolic if $G(\Sigma)$ is indefinite with negative inertia index equal to $1 ; \Sigma$ is said to be superhyperbolic if its negative inertia index is greater than $1 ; \Sigma$ is said to be admissible if $\Sigma$ contains no parabolic subdiagrams and is not superhyperbolic.

Table 1 contains the list of elliptic and connected parabolic Coxeter diagrams in their standard notation. In [20, Table 3] one finds a list of Lannér diagrams. Notice that the order of a Lannér diagram cannot be greater than 5; moreover, there are only finitely many Lannér diagrams of order greater than 3. In Table 2 we reproduce a list of Lannér diagrams of orders 4 and 5 and introduce notation for them.

2. It is convenient to describe Coxeter polytopes via their Coxeter diagrams. Let $P$ be a Coxeter polytope with facets $f_{1}, \ldots, f_{r}$. The Coxeter diagram $\Sigma(P)$ of $P$ is a diagram 
TABle 1. Coxeter diagrams. The left column lists connected elliptic Coxeter diagrams. The right column lists connected parabolic Coxeter diagrams

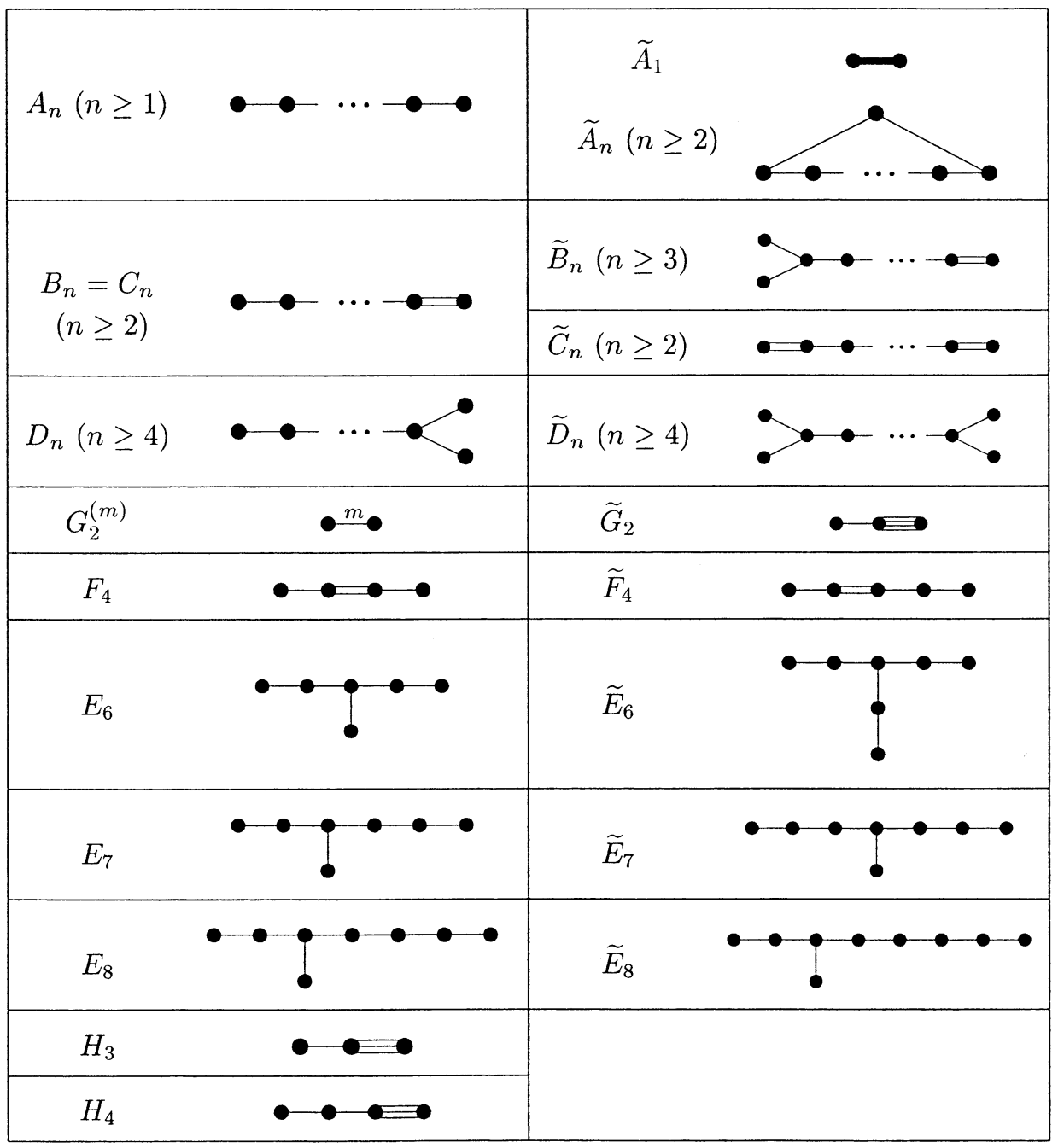

with nodes $v_{1}, \ldots, v_{r}$; nodes $v_{i}$ and $v_{j}$ are not joined if $f_{i}$ is orthogonal to $f_{j} ; v_{i}$ and $v_{j}$ are joined by an edge with weight

$$
w_{i j}= \begin{cases}\cos \frac{\pi}{k} & \text { if } f_{i} \text { and } f_{j} \text { form a dihedral angle } \frac{\pi}{k}, \\ 1 & \text { if } f_{i} \text { is parallel to } f_{j}, \\ \cosh \rho & \text { if } f_{i} \text { and } f_{j} \text { diverge and } \rho \text { is the distance between } f_{i} \text { and } f_{j} .\end{cases}
$$

If $\Sigma=\Sigma(P)$, then $G(\Sigma)$ coincides with the Gram matrix of the outward unit normals to the facets of $P$.

It was shown in [19] that if $\Sigma=\Sigma(P)$ is the Coxeter diagram of a $d$-dimensional compact hyperbolic polytope $P$, then $\Sigma$ is an admissible connected hyperbolic diagram with positive inertia index equal to $d$. In particular, $\Sigma$ has no bold edges and parabolic subdiagrams. The elliptic subdiagrams of $\Sigma$ are in one-to-one correspondence with the 
TABLE 2. Lannér diagrams of orders 4 and 5. Open nodes are black, doubly open nodes are encircled. The superscript equals the order of the diagram
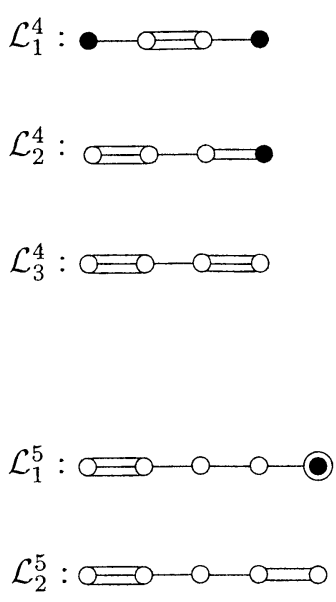
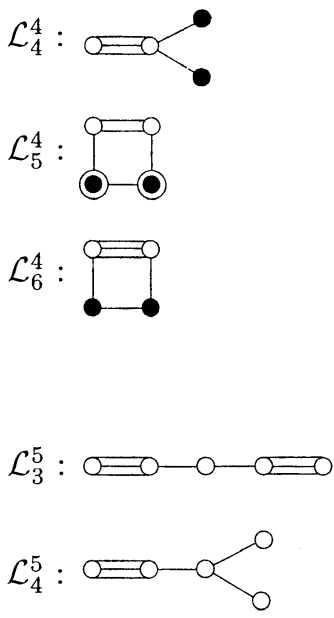
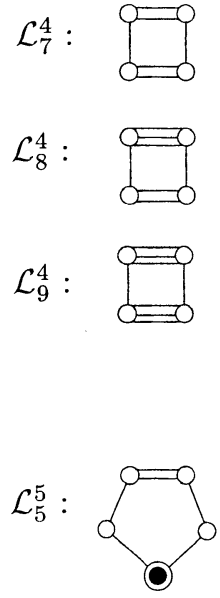

faces of $P$ : a $k$-face $F$ corresponds to the elliptic subdiagram $\Sigma_{F}$ of order $d-k$ whose nodes correspond to the facets containing $F$.

3. Given a Coxeter diagram $\Sigma$ it is easy to check whether or not it is superhyperbolic. However, when the order of the diagram is big, the computation of the signature could be difficult. When the diagram is the union of two subdiagrams either joined by a single edge or having only one node in common, there is a more effective way to determine if such a diagram is superhyperbolic $[18$.

Suppose $T$ is a subdiagram of $\Sigma$ such that $\operatorname{det}(\Sigma \backslash T) \neq 0$. A local determinant of $\Sigma$ on $T$ is $\operatorname{det}(\Sigma, T)=\frac{\operatorname{det}(\Sigma)}{\operatorname{det}(\Sigma \backslash T)}$.

Proposition 1.1 ([18, Prop.12]). If a Coxeter diagram $\Sigma$ consists of subdiagrams $\Sigma_{1}$ and $\Sigma_{2}$ that intersect in a single node $v$, then

$$
\operatorname{det}(\Sigma, v)=\operatorname{det}\left(\Sigma_{1}, v\right)+\operatorname{det}\left(\Sigma_{2}, v\right)-1 .
$$

Proposition 1.2 ([18, Prop. 13]). If a Coxeter diagram $\Sigma$ consists of non-intersecting subdiagrams $\Sigma_{1}$ and $\Sigma_{2}$ joined by a single edge $v_{1} v_{2}$, then

$$
\operatorname{det}\left(\Sigma,\left\langle v_{1}, v_{2}\right\rangle\right)=\operatorname{det}\left(\Sigma_{1}, v_{1}\right) \operatorname{det}\left(\Sigma_{2}, v_{2}\right)-w_{12}^{2},
$$

where $w_{12}$ is the weight of the edge $v_{1} v_{2}$.

Proposition 1.3 ([18, Prop. 15]). Suppose that a Coxeter diagram $\Sigma$ consists of two non-intersecting hyperbolic subdiagrams $\Sigma_{1}$ and $\Sigma_{2}$ joined by a single edge $v_{1} v_{2}$ such that $\Sigma_{1} \backslash v_{1}$ and $\Sigma_{2} \backslash v_{2}$ are elliptic. Assume also that one of the following conditions holds:

1) $v_{1} v_{2}$ is a simple edge and $\operatorname{det}\left(\Sigma_{1}, v_{1}\right) \operatorname{det}\left(\Sigma_{2}, v_{2}\right)>\frac{1}{4}$;

2) $v_{1} v_{2}$ is a double edge, and $\operatorname{det}\left(\Sigma_{1}, v_{1}\right) \operatorname{det}\left(\Sigma_{2}, v_{2}\right)>\frac{1}{2}$.

Then $\Sigma$ is superhyperbolic.

In [18, Table 2] one can find some useful determinants. When we need to check if a certain diagram is superhyperbolic, we use Propositions 1.11 .3 and Table 2 of [18] without further stipulation. We also use the fact that local determinants of Lannér diagrams of orders 4 and 5 on their open nodes (see Table 2 and the definition of an open 
node below) do not exceed 0.95 - this can be checked directly. In particular, we use the following consequence of Proposition 1.2 .

Proposition 1.4. Suppose $\Sigma$ consists of two disjoint Lannér diagrams $L_{1}$ and $L_{2}$, each of order 5 , joined by a single non-dashed edge $v_{1} v_{2}$ such that $L_{i} \backslash v_{i}$ (where $v_{i} \in L_{i}$, $i=1,2)$ are of type $H_{4}$ or $F_{4}$. Then $\Sigma$ is superhyperbolic.

4. Suppose $\Sigma$ is an abstract Coxeter diagram and $v$ is a node of $\Sigma$. Suppose we can add a node $x$ to $\Sigma$ so that $x \notin \Sigma, x$ is joined with $v$ in $\langle\Sigma, x\rangle$, and $x$ belongs to neither a Lannér nor a parabolic subdiagram of $\langle\Sigma, x\rangle$. Then $v$ is called an open node of $\Sigma$.

A node $v$ is said to be doubly open if a node $x$ can be added to $\Sigma$ so that $x$ be joined with $v$ in $\langle\Sigma, x\rangle$ and $x$ be open in $\langle\Sigma, x\rangle$.

Notice that for any non-open node $v$ of a Lannér diagram $\Sigma$ and any node $x \notin \Sigma$ joined with $v$ there exists a non-elliptic subdiagram $M \subset\langle\Sigma, x\rangle$ containing both $x$ and $v$. Indeed, if $x$ is not joined with $\Sigma \backslash v$, the assertion is obvious. When we add edges joining $x$ with $\Sigma \backslash v$, non-elliptic subdiagrams remain non-elliptic.

In [8, Table 2] Esselmann listed all Lannér diagrams of orders 4 and 5 containing open nodes. In Table 2, the open nodes are black, and the doubly open nodes are encircled.

As a direct consequence of the classification of Lannér diagrams, we have

Proposition 1.5. Any Lannér diagram of order 4 contains at least two subdiagrams of type $\mathrm{H}_{3}$ or $\mathrm{B}_{3}$.

Proposition 1.6 ([18, Prop. 2]). Any two Lannér subdiagrams of the diagram $\Sigma$ of a hyperbolic Coxeter polytope are joined by at least one edge.

This is obvious: if it is not true, then $\Sigma$ is a superhyperbolic diagram.

1.2. Gale diagrams and diagrams of missing faces. 1. As was shown in [19, Corollary to Th. 3.1], a compact hyperbolic Coxeter polytope is simple (i.e., a $k$-face of a $d$-dimensional polytope belongs to exactly $d-k$ facets). Now we want to list some combinatorial properties of simple polytopes. For brevity, a $d$-dimensional polytope will be called a " $d$-polytope".

Each combinatorial type of a simple $d$-polytope can be represented by its Gale diagram. It consists of $d+k$ points $a_{1}, \ldots, a_{d+k}$ on the $(k-2)$-dimensional sphere $\mathbb{S}^{k-2} \subset \mathbb{R}^{k-1}$ centered at the origin. Each $a_{i}$ corresponds to a facet $f_{i}$ of $P$. The combinatorial type of a simple convex polytope can be read off its Gale diagram as follows: for any subset $J \subset\{1, \ldots, d+k\}$ the intersection of the faces $\left\{f_{j} \mid j \in J\right\}$ is a face of $P$ if and only if the origin is an interior point of $\operatorname{conv}\left\{a_{j} \mid j \notin J\right\}$ (where conv $X$ is the convex hull of the set $X)$.

A set of points $a_{1}, \ldots, a_{d+k} \in \mathbb{S}^{k-2}$ is the Gale diagram of a $d$-polytope $P$ with $d+k$ facets if and only if each open half-space $H^{+}$of $\mathbb{R}^{k-1}$ bounded by a hyperplane passing through the origin contains at least two of the points $a_{1}, \ldots, a_{d+k}$.

Two Gale diagrams are said to be isomorphic if the corresponding polytopes are combinatorially equivalent.

Let $P$ be a simple polytope. The facets $f_{1}, \ldots, f_{m}$ of $P$ form a missing face if $\bigcap_{i=1}^{m} f_{i}=$ $\emptyset$, but any proper subset of the facets $\left\{f_{1}, \ldots, f_{m}\right\}$ has a non-empty intersection. Clearly, any set of facets with an empty intersection contains at least one missing face. Whence:

Lemma 1.1. Let $P$ be a simple d-polytope with $d+k$ facets, let $a_{1}, \ldots, a_{d+k} \in \mathbb{S}^{k-2}$ be the Gale diagram of $P$, and let $H^{+}$be an open half-space bounded by a hyperplane passing through the origin. Then $H^{+}$contains a set $I \subset\left\{a_{1}, \ldots, a_{d+k}\right\}$ corresponding to a missing face of $P$. 
2. When $k=2$, the Gale diagram of $P$ is 1 -dimensional, i.e., the points $a_{i}$ lie on a zero-dimensional unit sphere. In other words, each point $a_{i}$ lies on the number line and coincides with either -1 or 1 . Whence:

Proposition $1.7([12,7])$. A simple d-polytope with $d+2$ facets is the direct product of two simplices $\Delta^{n-k} \times \Delta^{k}$ (where $0 \leq k \leq[n / 2]$ and $\Delta^{m}$ stands for an m-simplex).

As was shown in [9, a compact Coxeter $d$-polytope with $d+2$ facets is either a simplicial prism or the product of two triangles. Coxeter prisms are listed in [13, the remaining Coxeter polytopes of this type (there are seven of them) can be found in 9 . We call those seven polytopes the Esselmann polytopes.

When $k=3$ the Gale diagram of $P$ is two-dimensional, i.e., the points $a_{i}$ lie on a unit circle.

A standard Gale diagram of a simple $d$-polytope with $d+3$ facets consists of labeled vertices $v_{1}, \ldots, v_{m}$ of a regular $m$-gon ( $m$ is odd) in $\mathbb{R}^{2}$ centered at the origin such that:

1) each label is a natural number and the sum of the labels equals $d+3$;

2) the sum of the labels of the vertices lying in a half-plane bounded by a line passing through the origin is at least 2 .

It is not difficult to check that each two-dimensional Gale diagram is isomorphic to some standard diagram (see, for example, [12]). Two $d$-polytopes with $d+3$ facets are combinatorially equivalent if and only if their standard Gale diagrams are congruent (i.e., coincide up to a motion of the plane).

When $k>3$, there is no definition of a standard Gale diagram. To describe the combinatorics of simple polytopes we shall use another kind of diagrams.

A diagram of missing faces is a finite set $D$ with a specified collection $\mathcal{M}_{D}$ of subsets of $D$ such that $M^{\prime} \not \subset M$ for any $M, M^{\prime} \in \mathcal{M}_{D}$. The order $|M|$ of $M$ is defined as the cardinality of $M$. The elements of $\mathcal{M}_{D}$ are called the missing faces of $D$.

A diagram $D_{1} \subset D$ of missing faces is a subdiagram of $D$ if for any $M \subset D_{1}$ we have that $M \in \mathcal{M}_{D_{1}}$ if and only if $M \in \mathcal{M}_{D}$.

It is convenient to visualize diagrams of missing faces as follows: for each element of $D$ mark a point (vertex) on the plane, and then encircle the set of points corresponding to a subset $M$ (i.e., draw a closed curve about the points) if and only if $M \in \mathcal{M}_{D}$.

3. With a simple polytope $P$ we associate a diagram of missing faces $D(P)$ as follows: the elements of $D(P)$ correspond to the facets of $P$; a set of elements is a missing face of $D(P)$ if and only if the corresponding facets form a missing face of $P$.

The combinatorial structure of $P$ can be recovered from $D(P)$ : a collection of facets has a non-empty intersection if and only if the corresponding subset of $D(P)$ contains no missing faces.

Lemma 1.2. Let $P$ be a simple polytope and $D(P)$ its diagram of missing faces. For any missing face $M \in \mathcal{M}_{D(P)}$ there is a missing face $M^{\prime} \in \mathcal{M}_{D(P)}$ such that $M \cap M^{\prime}=\emptyset$.

Proof. The Gale diagram $G(P)$ of $P$ consists of several points on the $d$-sphere $\mathbb{S}^{d}$. Let $\bar{M}$ be the points of $G(P)$ corresponding to the elements of $M$. Since $M$ is a missing face, $\operatorname{conv}(G(P) \backslash \bar{M})$ does not contain the origin. In other words, there is a hyperplane $H$ passing through the origin with a half-space $H^{+}$containing $G(P) \backslash \bar{M}$. Let $H^{-}$be the other half-space relative to $H$. By Lemma 1.1. $H^{-}$contains the points corresponding to some missing face. Since those points belong to $\bar{M}$ and a missing face cannot contain another missing face, we have that $H$ separates the points of $\bar{M}$ from the other points of $G(P)$, i.e., $H^{-} \cap G(P)=\bar{M}$. By Lemma1.1, $H^{+}$contains a subset of points corresponding to a missing face of $M^{\prime}$. Clearly, $M$ does not intersect $M^{\prime}$, which proves the lemma.

We also need the following two results. 
Proposition 1.8 ([8, Lemma 1.6]). Let $P$ be a simple polytope and $f$ a facet of $P$. Let $\left\{f_{1}, \ldots, f_{k}\right\}$ be the set of the facets of $P$ different from $f$ and such that $f_{i} \cap f \neq 0$. Set $f_{i}^{\prime}=f_{i} \cap f$ for each $i=1, \ldots, k$, and for any subset $G \subseteq\left\{f_{1}, \ldots, f_{k}\right\}$, set $G^{\prime}=$ $\left\{f_{i}^{\prime} \mid f_{i} \in G\right\}$.

Then $G^{\prime}$ is a missing face of the facet $f$ if and only if

1) either $\{f\} \cup G$ is a missing face of $P$, or

2) $G$ is a missing face of $P$ and $G$ contains no proper subsets $G_{0}$ such that $\{f\} \cup G_{0}$ is a missing face of $P$.

Proposition 1.9 ([8, Lemma 1.9]). For any facet $f$ of a simple polytope $P$ there is a missing face of $P$ containing $f$.

1.3. Lannér diagrams and missing faces. Let $P$ be a compact Coxeter polytope in $\mathbb{H}^{n}, \Sigma(P)$ its Coxeter diagram, and $L$ a Lannér subdiagram of $\Sigma(P)$. By the definition of Lannér subdiagrams, the facets corresponding to $L$ form a missing face of $P$ (and any missing face of $P$ corresponds to some Lannér subdiagram of $\Sigma(P)$ ). Thus, the diagram of missing faces $D(P)$ can easily be recovered from $\Sigma(P)$ : in $\Sigma(P)$, encircle all Lannér subdiagrams and remove all edges.

In the same way we construct a diagram of missing faces $D(\Sigma)$ for any admissible Coxeter diagram $\Sigma$.

The correspondence "Lannér diagram $\longleftrightarrow$ missing face" shows in particular that a compact hyperbolic Coxeter polytope has no missing faces of order greater than 5 .

1.4. Faces of Coxeter polytopes. Let $P$ be a compact hyperbolic Coxeter $d$-polytope, $\Sigma$ its Coxeter diagram, and $S_{0}$ an elliptic subdiagram of $\Sigma$. As was shown in [19, Th. 3.1], $S_{0}$ corresponds to a face of $P$ of dimension $d-\left|S_{0}\right|$. Denote that face $P\left(S_{0}\right)$. It is an acute-angled polytope [2], but it need not be a Coxeter polytope. R. Borcherds obtained the following sufficient condition for $P\left(S_{0}\right)$ to be a Coxeter polytope.

Proposition 1.10 ([4, Example 5.6]). Let $P$ be a compact hyperbolic Coxeter polytope with Coxeter diagram $\Sigma$, and $S_{0}$ an elliptic subdiagram of $\Sigma$ having no connected components of types $A_{n}$ and $D_{5}$. Then $P\left(S_{0}\right)$ is a Coxeter polytope.

The facets of $P\left(S_{0}\right)$ correspond to those nodes of $\Sigma$ that form elliptic subdiagrams with $S_{0}$. The dihedral angles of $P\left(S_{0}\right)$ can be determined using the following result of D. Allcock.

Let $a$ and $b$ be the facets of $P\left(S_{0}\right)$ determined by facets $A$ and $B$ of $P$, i.e., $a=$ $A \cap P\left(S_{0}\right)$ and $b=B \cap P\left(S_{0}\right)$. Let $v_{A}$ and $v_{B}$ be the nodes of $\Sigma$ corresponding to $A$ and $B$. The angles of $P\left(S_{0}\right)$ can now be found as follows.

Proposition 1.11 ([1, Th. 2.2]). Under the assumptions of Proposition 1.10,

(1) If neither $v_{A}$ nor $v_{B}$ is joined with $S_{0}$, then $\angle a b=\angle A B$.

(2) If exactly one of the nodes $v_{A}$ and $v_{B}$ is joined with $S_{0}$, say, via the connected component $S_{0}^{i}$, then

(a) if $A \perp B$, then $a \perp b$;

(b) if $v_{A}$ and $v_{B}$ are joined by a simple edge and adjoining $v_{A}$ and $v_{B}$ to $S_{0}^{i}$ yields a diagram of type $B_{k}$ (resp., $D_{k}, E_{8}$, or $H_{4}$ ), then $\angle a b=\pi / 4$ (resp., $\pi / 4, \pi / 6$ or $\pi / 10)$;

(c) otherwise, $a$ and $b$ do not intersect.

(3) If $v_{A}$ and $v_{B}$ are joined with different components of $S_{0}$, then

(a) if $A \perp B$, then $a \perp b$;

(b) otherwise, $a$ and $b$ do not intersect. 
(4) If $v_{A}$ and $v_{B}$ are joined with the same connected component of $S_{0}$, say $S_{0}^{i}$, then (a) if $A \perp B$ and $S_{0}^{i} \cup\{A, B\}$ is of type $E_{6}$ (resp., $E_{8}$ or $F_{4}$ ), then $\angle a b=\pi / 3$ (resp., $\pi / 4$ or $\pi / 4)$;

(b) otherwise, $a$ and $b$ do not intersect.

We shall say that $w \in \Sigma$ is a neighbor of $S_{0}$ if $w$ is joined with $S_{0}$ by an edge. A neighbor $w$ is said to be good if $\left\langle S_{0}, w\right\rangle$ is an elliptic diagram, and bad otherwise. Let $\bar{S}_{0}$ be the subdiagram of $\Sigma$ consisting of the nodes corresponding to the facets of $P\left(S_{0}\right)$. Then $\bar{S}_{0}$ is spanned by the good neighbors of $S_{0}$ and by the nodes which are not neighbors of $S_{0}$. If $P\left(S_{0}\right)$ is a Coxeter polytope, denote its diagram $\Sigma_{S_{0}}$. By a simple edge we understand a 1-fold edge. By an empty edge we understand two nodes which are not joined. By an ordinary edge we understand non-dashed edges, including empty ones.

For each node $v \in \Sigma$ which is not a bad neighbor of $S_{0}$ (i.e., it belongs to $\bar{S}_{0}$ ) the corresponding node of $\Sigma_{S_{0}}$ will be denoted $\widetilde{v}$.

In the case when $\Sigma_{S_{0}}$ does not differ from $\bar{S}_{0}$ we shall view $\Sigma_{S_{0}}$ as a subdiagram of $\Sigma$, and, accordingly, identify $v$ and $\widetilde{v}$.

Corollary 1.1. Under the assumptions of Proposition 1.10 ,

(a) If $S_{0}$ is of type $H_{4}, F_{4}, G_{2}^{(m)}$ for $m \geq 6$, or any other diagram with no good neighbors, then $\bar{S}_{0}=\Sigma_{S_{0}}$.

(b) If $S_{0}$ is of type $H_{3}$, then $\bar{S}_{0}$ can be obtained from $\Sigma_{S_{0}}$ by replacing some dashed edges by ordinary ones.

(c) If $S_{0}$ is of type $G_{2}^{(5)}$, then $\bar{S}_{0}$ can be obtained from $\Sigma_{S_{0}}$ by replacing some edges labeled by 10 with simple edges, and some dashed edges with ordinary ones.

(d) If $S_{0}$ is of type $B_{n}, n \geq 3$, then $\bar{S}_{0}$ can be obtained from $\Sigma_{S_{0}}$ by replacing some double edges by simple ones, and some dashed edges by ordinary ones.

(e) If $S_{0}$ is of type $B_{2}=G_{2}^{(4)}$, then $\bar{S}_{0}$ can be obtained from $\Sigma_{S_{0}}$ by replacing some double edges by simple ones, and some dashed edges by ordinary or empty edges.

The corollary follows directly from Proposition 1.11. We just remark that all neighbors of the diagrams mentioned in (a) are bad.

Here is another direct consequence of Proposition 1.11.

Corollary 1.2. Under the assumptions of Proposition 1.10, let $S_{1} \subset \Sigma_{S_{0}}$ be a subdiagram of type $G_{2}^{(m)}$, where $m \neq 4,10$, and let $S_{1}^{\prime}$ be the corresponding subdiagram $\bar{S}_{0}$. Then no node of $S_{1}^{\prime} \subset \Sigma$ is a good neighbor of $S_{0}$.

In particular, any subdiagram $S_{2} \subset \Sigma_{S_{0}}$ of type $F_{4}, H_{4}, H_{3}$ or $G_{2}^{(m)}$, where $m \neq 4,10$, corresponds to a subdiagram of $\bar{S}_{0}$ of the same type. $\bar{S}_{0}$.

Any Lannér subdiagram $L \subset \Sigma_{S_{0}}$ of order 5 corresponds to a Lannér subdiagram of

Lemma 1.3. Suppose that $S_{0}$ is an elliptic subdiagram and $\left|S_{0}\right|<d$. Then $S_{0}$ has at most $|\Sigma|-d-1$ bad neighbors. In particular, if $P$ has $d+4$ facets, then any elliptic subdiagram of $\Sigma$ of order less than d has at most three bad neighbors.

Proof. The lemma follows from the fact that a $k$-polytope has at least $k+1$ facets.

Lemma 1.4. Let $S \subset \Sigma$ be an elliptic subdiagram containing no components of types $A_{n}$ and $D_{5}$, and let a be a bad neighbor of $S$. Then a is joined with each Lannér subdiagram of $\bar{S}$.

This is a direct consequence of Proposition 1.6. 


\section{Admissible Coxeter diagrams without Small Lannér subdiagrams}

A Lannér diagram (or a missing face) $L$ is said to be small if $|L|<5$. A missing face $M$ is said to be large if $|M|>5$.

Lemma 2.1. Let $\Sigma$ be a connected admissible Coxeter diagram without small Lannér subdiagrams. Suppose that each node of $\Sigma$ belongs to some Lannér subdiagram of $\Sigma$. Then $|\Sigma| \leq 10$. If $|\Sigma|=10$, then $\Sigma$ is one of the three diagrams shown in Figure 1 .

If, in addition, $\operatorname{det}(\Sigma)=0$, then $\Sigma=\Theta_{1}$ (see Figure 1).

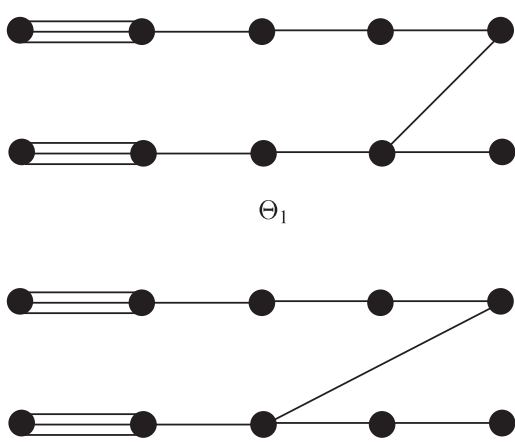

$\Theta_{2}$

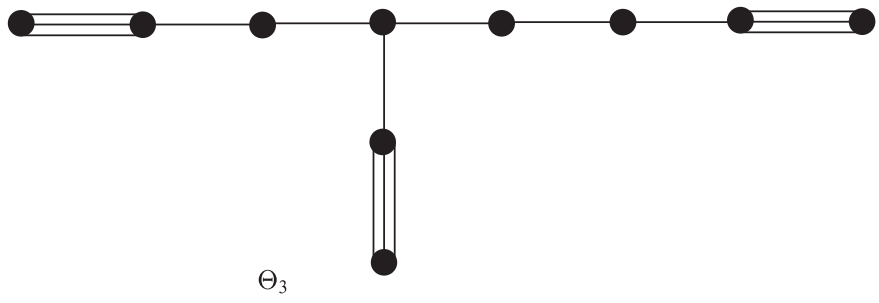

FiguRE 1. Diagrams without small Lannér subdiagrams

Proof. Since $\Sigma$ is connected and does not contain small Lannér subdiagrams, any edge of $\Sigma$ is either simple, or double, or triple. Consider two cases: either $\Sigma$ contains $\mathcal{L}_{5}^{5}$ or it does not (for notation, see Table 2).

Case 1. Suppose that $\Sigma$ contains $\mathcal{L}_{5}^{5}$. It is clear that only the open node of $\mathcal{L}_{5}^{5}$ can be joined with some other nodes of $\Sigma$; otherwise $\Sigma$ would contain either a parabolic subdiagram of type $\widetilde{B}_{4}$ or $\widetilde{B}_{5}$, or a small Lannér subdiagram. For the same reason, $\Sigma \backslash \mathcal{L}_{5}^{5}$ is linear and contains no double edges. Since each node of $\Sigma$ belongs to some Lannér subdiagram, $\Sigma \backslash \mathcal{L}_{5}^{5}$ contains a triple edge, and for $|\Sigma|>9$ such a diagram either contains a small Lannér subdiagram or is superhyperbolic.

Case 2. Suppose that $\Sigma$ does not contain $\mathcal{L}_{5}^{5}$.

- For any triple edge of $\Sigma$, one of its nodes is a leaf of $\Sigma$ and the valency of the other node is two.

Proof. This follows immediately from the absence of small Lannér subdiagrams.

- $\Sigma$ has no double edges. 
Proof. Let $v_{1} v_{2}$ be a double edge of $\Sigma$. Suppose that $v_{1}$ is a leaf of $\Sigma$. By assumption, $v_{1}$ belongs to some Lannér diagram $L$. It is clear that $L$ contains $v_{2}$ and is a linear Lannér diagram. Let $v$ be a node of $\Sigma \backslash L$ joined with $L$. Then $\langle L, v\rangle$ contains either a parabolic or a small Lannér subdiagram.

Thus, neither $v_{1}$ nor $v_{2}$ is a leaf of $\Sigma$. Hence $\Sigma$ contains edges of the form $v_{0} v_{1}$ and $v_{2} v_{3}$ (where $v_{0} \neq v_{3}$, since otherwise $\left\langle v_{0}, v_{1}, v_{2}\right\rangle$ would contain a Lannér or a parabolic diagram). These edges are simple; otherwise $\Sigma$ would contain a parabolic or a small Lannér subdiagram. Therefore, $\left\langle v_{0}, v_{1}, v_{2}, v_{3}\right\rangle$ is of type $F_{4}$. If $v_{4}$ is an arbitrary node of $\Sigma$ joined with $\left\langle v_{0}, v_{1}, v_{2}, v_{3}\right\rangle$, then either $\left\langle v_{0}, v_{1}, v_{2}, v_{3}, v_{4}\right\rangle$ is of type $\mathcal{L}_{5}^{5}$ or it contains a parabolic or a small Lannér subdiagram, contrary to the assumption.

- $\Sigma$ is a tree.

Proof. The two preceding results imply that any minimal cycle of $\Sigma$ is a parabolic subdiagram of type $\widetilde{A}_{n}$.

Now we remove from $\Sigma$ all leaves that belong to triple edges and denote the obtained diagram $\Sigma^{\prime}$. As was shown above, $\Sigma^{\prime}$ is a tree with simple edges. All such trees without parabolic subdiagrams are elliptic diagrams of type $A_{n}, D_{n}, E_{6}, E_{7}$, or $E_{8}$.

Next we attach triple edges to some leaves of $\Sigma^{\prime}$. If $\Sigma^{\prime}=A_{n}$ or $D_{n+1}$ for $n \geq 9$, then $\Sigma$ has a node that does not belong to any Lannér subdiagram. In the remaining cases either $|\Sigma|<10$, or $\Sigma$ is superhyperbolic, or $\Sigma$ is one of the diagrams in Figure 1.

A direct calculation shows that $\operatorname{det} \Sigma=0$ only when $\Sigma=\Theta_{1}$.

Corollary 2.1. The Coxeter diagram $\Sigma(P)$ of any compact Coxeter polytope $P$ contains a small Lannér subdiagram.

Proof. Suppose that $\Sigma(P)$ does not contain small Lannér diagrams. By Lemma 1.2 , $\Sigma(P)$ contains two disjoint Lannér subdiagrams $L_{1}$ and $L_{2}$ of order 5 , and therefore, $|\Sigma(P)| \geq 10$. The subdiagram $\left\langle L_{1}, L_{2}\right\rangle$ is connected because otherwise it would be superhyperbolic. It follows from Lemma 2.1 that $\Sigma(P)$ is one of the diagrams from Figure 1 None of those diagrams is the Coxeter diagram of a compact polytope: in each of the three cases, $\Sigma(P)$ contains a Lannér subdiagram that intersects any other Lannér subdiagram of $\Sigma(P)$, which is impossible by Lemma 1.2 .

\section{LIFTINGS}

Let $D$ be a diagram of missing faces, and $\Sigma$ an admissible Coxeter diagram. The diagram $\Sigma$ is called a 0 -lifting of $D$ if there is a bijection $\phi: D \rightarrow \Sigma$ such that $M \in \mathcal{M}_{D}$ if and only if $\phi(M)$ is a Lannér subdiagram; $\phi$ is called a lifting bijection.

$\Sigma$ is called a $k$-lifting of $D(k \in \mathbb{N})$ if $\Sigma$ contains a subset $A$ of "additional nodes" such that:

1) $|A|=k$;

2) there is an injection $\phi: D \rightarrow \Sigma$ sending $D$ bijectively to $\Sigma \backslash A$; $\phi$ is called a lifting injection;

3) for any Lannér diagram $L \subset \Sigma$ the set $\phi^{-1}(L \backslash A)$ contains a missing face of $D$;

4) for any missing face $M \in \mathcal{M}_{D}$ there is a Lannér subdiagram $L \subset\langle\phi(M), A\rangle$ containing $\phi(M)$;

5) for any set $\left\{a_{1}, \ldots, a_{r}\right\} \subset A$ the subdiagram $\Sigma \backslash\left\{a_{1}, \ldots, a_{r}\right\}$ is not a $(k-r)$-lifting of $D$.

We remark that in 8 a 0 -lifting of $D$ was called a "hyperbolic realization" of $D$. Also, a 0 -lifting satisfies the general definition of a $k$-lifting. When the value of $k$ is not important, we write "lifting" instead of " $k$-lifting". 
Let $\Sigma$ be a lifting of $D$, and $L \subset \Sigma$ a Lannér subdiagram such that for any missing face $M \in \mathcal{M}_{D}$ we have $L \nsubseteq\langle\phi(M), A\rangle$. We then say that $L$ is an additional Lannér subdiagram of the lifting $\Sigma$.

Let $D$ be an abstract diagram of missing faces, $\Sigma$ an abstract Coxeter diagram, and $\phi: D \rightarrow \Sigma$ an injection. Let $\Sigma^{\prime} \subset \Sigma$ be a non-elliptic subdiagram not containing $\phi(M)$ for any $M \in \mathcal{M}_{D}$. We then say that $\Sigma^{\prime}$ is a conflicting subdiagram; in that case $\Sigma$ (with the given injection $\phi$ ) is not a lifting of $D$.

Notice that $\phi(M)$ is a subset of the set of nodes of $\Sigma$. When we mean the Coxeter diagram spanned by the nodes of $\phi(M)$, we write $\langle\phi(M)\rangle$, in accordance with the notation introduced in 1.1 .

Lemma 3.1. Let $D$ be a diagram of missing faces, and $D_{1} \subset D$ a subdiagram of it. If $\Sigma$ is a $k$-lifting of $D$, then $\Sigma$ contains a subdiagram $\Sigma_{1}$ which is a $k_{1}$-lifting of $D_{1}$ for some $k_{1} \leq k$.

Proof. Let $A$ be the set of additional nodes of $\Sigma$. Consider $\left.\phi\right|_{D_{1}}$ as a lifting injection of $D_{1}$. By the definition of lifting, $\left\langle\phi\left(D_{1}\right), A\right\rangle$ contains some $k_{1}$-lifting of $D_{1}$ with $k_{1} \leq k$.

The proof of the following lemma is based on a repeated application of Proposition 1.8.

Lemma 3.2. Let $P \subset \mathbb{H}^{d}$ be a simple hyperbolic Coxeter polytope, and $f$ an $m$-face of it. Let $D(f)$ be the diagram of missing faces of $f$. Then $\Sigma(P)$ contains a subdiagram $\Sigma_{0}$ which is a k-lifting of $D(f)$ for some $k \leq d-m$.

Proof. Let $f_{1}, \ldots, f_{d-m}$ be the facets of $P$ containing $f$. Set $F_{1}=f_{1}$ and define inductively $F_{i}=F_{i-1} \cap f_{i}$. It is clear that $F_{i}$ is a facet of $F_{i-1}$ and $F_{d-m}=f$.

When $m=d$, the lemma is trivial. Assuming that the assertion holds for any face of dimension greater than $m$ we shall prove it for the $m$-face $f$. By the induction assumption, $\Sigma(P)$ contains a subdiagram $\Sigma_{r-1}$ which is a $k$-lifting of $D\left(F_{d-m-1}\right)$, where $k \leq d-$ $(m+1)$. The following claim then completes the proof.

Claim. Either $\Sigma_{r-1}$ contains a $k$-lifting of $f$ or $\left\langle\Sigma_{r-1}, v_{d-m}\right\rangle$ contains a $(k+1)$-lifting of $f$, where $v_{d-m}$ is the node of $\Sigma(P)$ corresponding to the facet $f_{d-m}$.

Proof of the claim. Let $\Pi_{1}, \ldots, \Pi_{s}$ be the facets of $F_{d-m-1}$. Denote by $J$ the set of indices $i$ such that $\Pi_{i} \cap f_{d-m} \neq \emptyset$ and set $\pi_{i}=\Pi_{i} \cap f_{d-m}, i \in J$. Then $\left\{\pi_{i}\right\}$ is the set of facets of $f=F_{d-m}$.

Let $\phi_{1}$ be a lifting injection for $F_{d-m-1}$; in particular, $\phi_{1}$ sends $\left\{\Pi_{i}\right\}$ to $\Sigma_{r-1} \backslash A$, where $A$ is the set of additional nodes. Denote by $\psi$ the map $\pi_{i} \rightarrow \Pi_{i}$ and consider $\phi=\phi_{1} \circ \psi$. Then $\phi$ is an injection from $\left\{\pi_{i}\right\}$ to $\Sigma \backslash A$. Let $\left\{\Pi_{i} \mid i \in I\right\}$ be a missing face of $D\left(F_{d-m}\right)$ (where $I \subset J$ is some indexing set). By Proposition 1.8, either $\left\{\pi_{i} \mid i \in I\right\}$ or $\left\{f_{d-m}\right\} \cup\left\{\pi_{i} \mid i \in I\right\}$ is a missing face of $D\left(F_{d-m-1}\right)$ for some $i$. This establishes condition 4) in the definition of lifting for either $\Sigma_{r-1}$ or $\left\langle\Sigma_{r-1}, v_{d-m}\right\rangle$. By the same proposition, if $\left\{\Pi_{i} \mid i \in K\right\}$ is not a missing face of $D\left(F_{d-m}\right)$, then neither $\left\{\pi_{i} \mid i \in K\right\}$ nor $\left\{f_{d-m}\right\} \cup\left\{\pi_{i} \mid i \in K\right\}$ is a missing face of $D\left(F_{d-m-1}\right)$, whence condition 3).

Thus, either $A$ or $A \cup\left\{f_{d-m}\right\}$ contains a set of additional nodes for some $k$-lifting of $D\left(F_{d-m-1}\right)$. Hence $k \leq d-(m+1)+1=d-m$, and the lemma is proved.

Corollary 3.1. Let $P \subset \mathbb{H}^{d}$ be a compact Coxeter polytope, and let $f$ be a face of $P$. Then $D(f)$ does not contain large missing faces. In particular, if $\operatorname{dim} f>4$, then $f$ is not a simplex.

Proof. By the definition of lifting, for any missing face $M$ of $D$ there is a Lannér subdiagram $L$ of $\Sigma$ such that $|L| \geq|M|$. Now the assertion follows from the fact that a Lannér diagram contains at most five nodes. 
Lemma 3.3. Let $D$ be a diagram of missing faces such that $|M|=5$ for any $M \in \mathcal{M}_{D}$. Then any lifting of $D$ is a 0-lifting.

Proof. Suppose that $D$ is a $k$-lifting, where $k>0$, and $\phi$ is a lifting injection. Remove from $D$ all additional nodes and denote the obtained diagram $D_{1}$. We shall show that $D_{1}$ is a 0 -lifting and $\phi$ is the lifting bijection. Indeed, conditions 2), 3), and 5) in the definition of $k$-lifting clearly hold.

For condition 4), consider an arbitrary missing face $M$ of $D$. By definition, $\phi(M)$ belongs to some Lannér subdiagram of $\Sigma$. Notice that the order of $\langle\phi(M)\rangle$ equals 5 . Since the order of a Lannér diagram is at most $5,\langle\phi(M)\rangle$ is a Lannér diagram.

Next we want to establish several properties of liftings which will be needed later.

Notation.

- Let $D$ be a diagram of missing faces and $N_{1}, \ldots, N_{r} \subset D$. We write $D=\bigcup_{i=1}^{r} N_{i}$ if for any node of $D$ there is a set $N_{i}, i \in\{1, \ldots, r\}$, containing that node.

- Let $D=\bigcup_{i=1}^{r} N_{i}$ be a diagram of missing faces such that $N_{i} \cap N_{j}=\emptyset$ for every $i \neq j$. Suppose that there is a $k \in\{1, \ldots, r\}$ such that $M \in \mathcal{M}_{D}$ if and only if $M=\bigcup_{t=i}^{i+k-1} N_{t}$ for some $i \in\{1, \ldots, r-k+1\}$. In this case we write $D=\left\lfloor N_{1}, N_{2}, \ldots, N_{r}\right\rfloor_{k}$.

- When we are interested only in the combinatorial type of $D$ rather than the actual subdiagrams $N_{i}$, we write $\left\lfloor\left|N_{1}\right|, \ldots,\left|N_{r}\right|\right\rfloor_{k}$. For example, $\lfloor 1,4,1,3\rfloor_{2}$ indicates the diagram

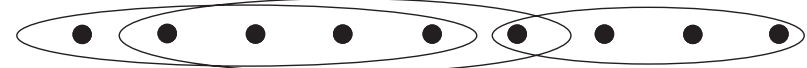

- If there is a $k \in\{1, \ldots, r\}$ such that $M \in \mathcal{M}_{D}$ if and only if $M=\bigcup_{t=i}^{i+k-1} N_{t}$ for some $i \in 1, \ldots, r$, where $t$ is taken modulo $r$, we write $D=\left(N_{1}, N_{2}, \ldots, N_{r}\right)_{k}$ or $\left(\left|N_{1}\right|, \ldots,\left|N_{r}\right|\right)_{k}$.

- We write $\Sigma \approx\left\lfloor N_{1}, N_{2}, \ldots, N_{r}\right\rfloor_{k}$ if $\Sigma$ contains no parabolic subdiagrams and the structure of Lannér subdiagrams of $\Sigma$ corresponds to the diagram of missing faces $\left\lfloor N_{1}, N_{2}, \ldots, N_{r}\right\rfloor_{k}$; i.e., $\Sigma$ consists of disjoint subdiagrams $L_{1}, \ldots, L_{r},\left|L_{i}\right|=\left|N_{i}\right|$, and $L \subset \Sigma$ is a Lannér subdiagram if and only if $L=\left\langle L_{i}, L_{i+1}, \ldots, L_{i+k-1}\right\rangle$ for some $i \in\{1, \ldots, r-k+1\}$.

Similarly, we use the notation

$$
\Sigma \approx\left\lfloor\left|N_{1}\right|, \ldots,\left|N_{r}\right|\right\rfloor_{k}, \quad \Sigma \approx\left(N_{1}, N_{2}, \ldots, N_{r}\right)_{k}, \quad \text { and } \quad \Sigma \approx\left(\left|N_{1}\right|, \ldots,\left|N_{r}\right|\right)_{k} .
$$

Proposition 3.1 ([8, Lemma 4.7]). Let $D=\lfloor M, N\rfloor_{1}$ be a diagram of missing faces with $|M| \geq 3$ and $|N| \geq 4$. Then $D$ has no 0-liftings.

Lemma 3.4. The diagram $\Theta_{1}$ of Figure 1 is the only lifting of $D=\lfloor 4,5\rfloor_{1}$. The additional node of this lifting is the leaf of $\Theta_{1}$ which does not belong to a triple edge.

Proof. Let $\Sigma$ be a lifting of $D=\left\lfloor M_{4}, M_{5}\right\rfloor_{1}$ with $\left|M_{5}\right|=5$ and $\left|M_{4}\right|=4$, and let $\phi$ be a lifting injection. Suppose that $\left\langle\phi\left(M_{4}\right)\right\rangle$ is a Lannér subdiagram of $\Sigma$. By Proposition 3.1, $\Sigma \approx\left\lfloor\phi\left(M_{4}\right), \phi\left(M_{5}\right)\right\rfloor_{1}$ is superhyperbolic. Hence $\left\langle\phi\left(M_{4}\right)\right\rangle$ is elliptic and, by condition 4), $\Sigma$ contains an additional node $a$ such that $\left\langle\phi\left(M_{4}\right), a\right\rangle$ is Lannér. Then $\left\langle a, \phi\left(M_{4}\right), \phi\left(M_{5}\right)\right\rangle$ satisfies conditions 1)-4) in the definition of lifting, and, by condition 5), $a$ is the only additional node and $\Sigma$ is a 1-lifting. By condition 3), the order of any Lannér subdiagram of $\Sigma$ is 5 . Since $|\Sigma|=\left|\left\langle a, \phi\left(M_{4}\right), \phi\left(M_{5}\right)\right\rangle\right|=10$, it follows from Lemma 2.1 that $\Sigma$ is one of the diagrams $\Theta_{1}, \Theta_{2}$, and $\Theta_{3}$ of Figure 1, By Lemma 1.2, $\Sigma$ contains two disjoint Lannér diagrams $\left\langle\phi\left(M_{4}\right), a\right\rangle$ and $\left\langle\phi\left(M_{5}\right)\right\rangle$, and therefore $\Sigma$ is either $\Theta_{1}$ or $\Theta_{2}$. 
It is not difficult to find the only lifting injection for $\Theta_{1}: a$ is the only leaf of $\Sigma$ which does not belong to a triple edge, and $\phi\left(M_{4}\right)$ consists of the remaining nodes in the bottom row (see Figure 1).

For $\Theta_{2}$ there are no lifting injections, since $\left\langle\phi\left(M_{4}\right)\right\rangle$ belongs to each Lannér subdiagram different from $\left\langle\phi\left(M_{5}\right)\right\rangle$, whereas $\Theta_{2}$ has no such quadruples of nodes.

Lemma 3.5. Let $D=N \cup\left\{x_{1}, x_{2}, x_{3}\right\}$ be a diagram of missing faces with $|N|=4$. If $\mathcal{M}_{D}=\left\{N \cup x_{1}, N \cup x_{2}, N \cup x_{3}\right\}$, then $D$ has no liftings.

Proof. Suppose that $\Sigma$ is a lifting of $D$ and $\phi$ is a lifting injection. By Lemma 3.3, $\Sigma$ is a 0-lifting. Consider $\Sigma_{i j}=\left\langle\phi\left(N \cup\left\{x_{i}, x_{j}\right\}\right)\right\rangle, i \neq j$. It is clear that $\Sigma_{i j} \approx\lfloor 1,4,1\rfloor_{2}$. By [8. Lemma 5.3], $\lfloor 1,4,1\rfloor_{2}$ is one of the following diagrams:

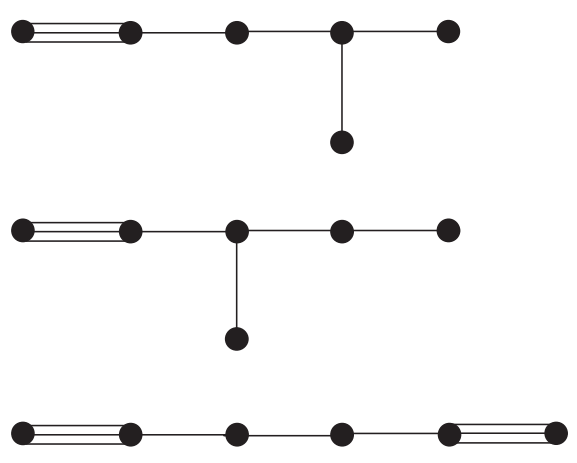

Viewing each of these subdiagrams as $\Sigma_{12}$ and trying to add $x_{3}$ to form $\Sigma_{13}$, we obtain in each case a conflicting subdiagram, which proves the lemma.

Lemma 3.6. The diagram $D=\lfloor 4,4\rfloor_{1}$ has no 0-liftings. Any 1-lifting of $D$ is one of the diagrams of Figure 2 .

Proof. By Proposition [3.1, $D$ has no 0-liftings. Let $\Sigma$ be a 1-lifting of $D, \phi$ a lifting injection, and $a$ the additional node.

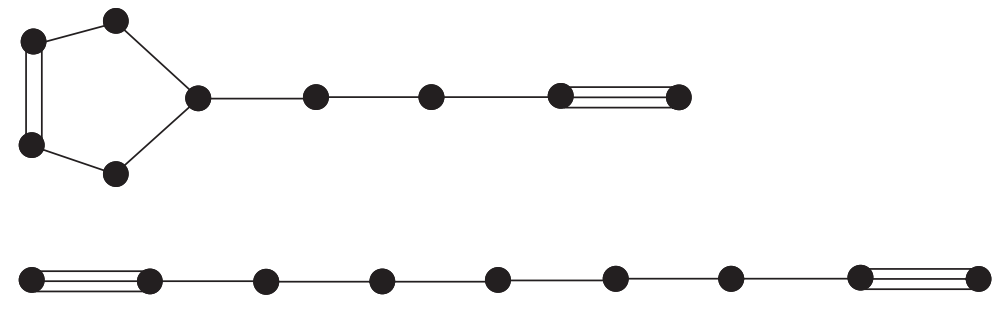

FIGURE 2. 1-liftings of $\lfloor 4,4\rfloor_{1}$

Let $M_{1}$ and $M_{2}$ be the missing faces of $D$. We may assume that the diagram $\left\langle\phi\left(M_{1}\right)\right\rangle$ is elliptic and the diagram $\left\langle\phi\left(M_{1}\right), a\right\rangle$ is Lannér. We consider two cases.

Case 1. Suppose that $\left\langle\phi\left(M_{2}\right)\right\rangle$ is a Lannér diagram. By Proposition 3.1. $\Sigma$ contains an additional Lannér subdiagram $L=\left\langle\phi\left(M_{1}\right), x\right\rangle$, where $x \in \phi\left(M_{2}\right)$. By Lemma 3.5, that additional subdiagram is unique. Thus,

$$
\Sigma \approx\left\lfloor a, \phi\left(M_{1}\right), x, \phi\left(M_{2}\right) \backslash x\right\rfloor_{2}=\lfloor 1,4,1,3\rfloor_{2},
$$

contrary to [8, Cor. 5.10]. 
Case 2. Suppose that both $\left\langle\phi\left(M_{1}\right)\right\rangle$ and $\left\langle\phi\left(M_{2}\right)\right\rangle$ are elliptic. Then $\left\langle\phi\left(M_{1}\right), a\right\rangle$ and $\left\langle\phi\left(M_{2}\right), a\right\rangle$ are Lannér. Notice that $a$ is an open node of $\left\langle\phi\left(M_{1}\right), a\right\rangle$, since otherwise $\Sigma$ would contain a conflicting Lannér subdiagram. Similarly, $a$ is an open node of $\left\langle\phi\left(M_{2}\right), a\right\rangle$. Hence each of the diagrams $\left\langle\phi\left(M_{1}\right), a\right\rangle$ and $\left\langle\phi\left(M_{2}\right), a\right\rangle$ coincides with $\mathcal{L}_{1}^{5}$ or $\mathcal{L}_{5}^{5}$.

If $\Sigma$ has an edge joining $\left\langle\phi\left(M_{1}\right)\right\rangle$ with $\left\langle\phi\left(M_{2}\right)\right\rangle$, then $\Sigma$ has a conflicting subdiagram. Thus, the subdiagrams $\left\langle\phi\left(M_{1}\right)\right\rangle$ and $\left\langle\phi\left(M_{2}\right)\right\rangle$ are not joined in $\Sigma$, and $\Sigma$ is one of the two diagrams of Figure 2 (if $\left\langle\phi\left(M_{1}\right), a\right\rangle=\left\langle\phi\left(M_{2}\right), a\right\rangle=\mathcal{L}_{5}^{5}$, then $\Sigma$ contains a parabolic subdiagram).

Let $P_{8}$ denote the only compact hyperbolic Coxeter 8-polytope with eleven facets (see [5, 8]), and let $\Sigma\left(P_{8}\right)$ be its Coxeter diagram (see Figure 3).

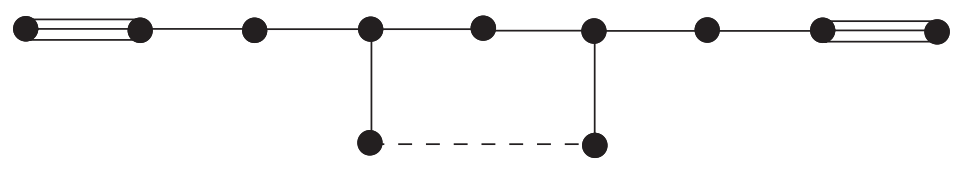

FIgURE 3. The only compact hyperbolic Coxeter 8-polytope with 11 facets

Lemma 3.7. Let $D=(4,4,2)_{1}$, and let $\Sigma$ be a 0 - or 1-lifting of $D$ that does not contain Lannér subdiagrams of order 3 . If the positive inertia index of $\Sigma$ is at most 8 , then $\Sigma=\Sigma\left(P_{8}\right)$.

Proof. By Lemmas 3.1 and 3.6, $D$ has no 0-liftings. Let $\Sigma$ be a 1-lifting of $D, \phi$ a lifting injection, and $a$ the additional node. Let $M_{1}, M_{2}$, and $M_{3}$ be the missing faces of $D$, where $\left|M_{1}\right|=\left|M_{2}\right|=4$ and $\left|M_{3}\right|=2$. Let $M_{3}=\left\langle u_{1}, u_{2}\right\rangle$. By Lemma 3.6. $\left\langle\phi\left(M_{1}\right), \phi\left(M_{2}\right), a\right\rangle$ is one of the two diagrams of Figure 2. Since $\Sigma$ contains only one additional node, the non-existence of Lannér subdiagrams of order 3 implies that $\left\langle\phi\left(M_{3}\right)\right\rangle$ is Lannér. Hence the order of any Lannér subdiagram of $\Sigma$ different from $\left\langle\phi\left(M_{3}\right)\right\rangle$ is 5 .

Suppose that $u_{1}$ does not belong to any additional Lannér subdiagram of $\Sigma$. Then $\left\langle\phi\left(M_{1}\right), \phi\left(M_{2}\right), u_{1}\right\rangle$ is elliptic of order 9, contrary to the assumption.

Thus $u_{1}$ and $u_{2}$ belong to some additional Lannér subdiagrams. The subdiagram $X_{1}=$ $\left\langle u_{1}, \phi\left(M_{1}\right), \phi\left(M_{2}\right), a\right\rangle$ is of order 10 and does not contain small Lannér subdiagrams. Moreover, each node of $X_{1}$ belongs to some Lannér diagram. By Lemma 2.1, $X_{1}$ is one of the diagrams $\Theta_{1}, \Theta_{2}$, and $\Theta_{3}$ of Figure1. Since the positive inertia index of $\Sigma$ is at most 8, we have $\operatorname{det}\left(X_{1}\right)=0$ and $X_{1}=\Theta_{1}$ (Lemma 2.1). By Lemma 3.6. $\left\langle\phi\left(M_{1}\right), \phi\left(M_{2}\right), a\right\rangle$ is a linear subdiagram of $\Theta_{1}$. Hence $u_{1}$ is the only leaf of $\Theta_{1}$ not incident to a triple edge. Similarly, $X_{2}=\left\langle u_{2}, \phi\left(M_{1}\right), \phi\left(M_{2}\right), a\right\rangle$ also coincides with $\Theta_{1}$. Moreover, $u_{1}$ and $u_{2}$ are joined with different nodes of $\left\langle\phi\left(M_{1}\right), \phi\left(M_{2}\right), a\right\rangle$, since otherwise the diagram $\left\langle\phi\left(M_{i}\right), a, u_{1}, u_{2}\right\rangle$ would be superhyperbolic. Thus $\Sigma=\Sigma\left(P_{8}\right)$ and the lemma is proved.

Lemma 3.8. Let $D=(1,4,1,3,1)_{2}$, and let $\Sigma$ be a 0 - or 1-lifting of $D$ that does not contain Lannér subdiagrams of order 3 . If the positive inertia index of $\Sigma$ is at most 8, then $\Sigma=\Sigma\left(P_{8}\right)$.

Proof. Let $\phi$ be a lifting injection. We use the following notation for the subdiagrams of $D:(1,4,1,3,1)_{2}=\left(v, N_{4}, u, N_{3}, w\right)_{2}$.

Suppose that $\left\langle\phi\left(N_{3}\right), \phi(u)\right\rangle$ is a Lannér diagram. Then

$$
\left\langle\phi(v), \phi\left(N_{4}\right), \phi(u), \phi\left(N_{3}\right)\right\rangle \approx\lfloor 1,4,1,3\rfloor_{2},
$$

which is impossible by [8, Cor. 5.10]. Hence $\left\langle\phi\left(N_{3}\right), \phi(u)\right\rangle$ is elliptic and $\Sigma$ cannot be a 0-lifting of $D$. 
Now assume that $\Sigma$ is a 1-lifting and $a$ is the only additional node of $\Sigma$. Then $X=\langle a, \phi(D \backslash\{w\})\rangle$ satisfies the assumptions of Lemma 2.1. Since $|X|=10, X$ is one of the diagrams $\Theta_{1}, \Theta_{2}$, and $\Theta_{3}$ of Figure 1 By assumption, the positive inertia index of $\Sigma$ is at most 8 and therefore $\operatorname{det}(X)=0$ and $X=\Theta_{1}$ :

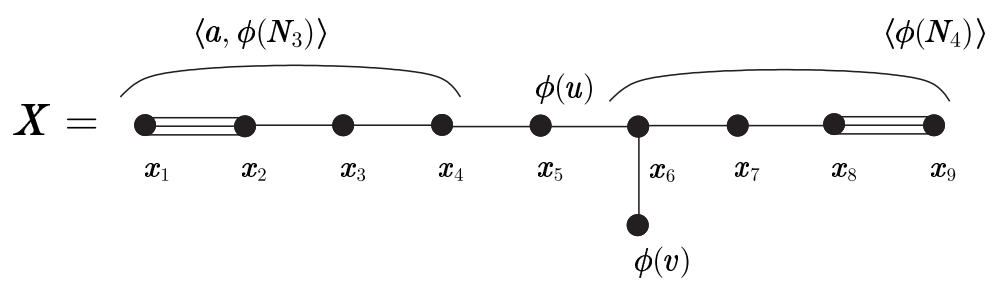

Consider the diagram $\langle X, \phi(w)\rangle$. Since $\left\langle\phi\left(N_{4}\right), \phi(w)\right\rangle$ is elliptic, $\phi(w)$ is not joined with $\left\langle x_{6}, x_{7}, x_{8}, x_{9}\right\rangle=\left\langle\phi\left(N_{4}\right)\right\rangle$. Furthermore, $\phi(w)$ is not joined with $\left\langle x_{1}, x_{2}\right\rangle$, otherwise $\left\langle x_{1}, x_{2}, x_{3}, \phi(w)\right\rangle$ would be conflicting. Hence, besides $\phi(v), \phi(w)$ may be joined only with $x_{3}, x_{4}$, or $x_{5}$. Any edge joining $w$ with $x_{3}, x_{4}, x_{5}$ is simple, since otherwise one of the diagrams $\left\langle x_{2}, x_{3}, x_{4}, \phi(w)\right\rangle,\left\langle x_{3}, x_{4}, x_{5}, \phi(w)\right\rangle$, and $\left\langle x_{4}, x_{5}, x_{6}, \phi(w)\right\rangle$ would be conflicting. To avoid parabolic subdiagrams, $\phi(w)$ must be joined with at most one of the nodes $x_{3}, x_{4}, x_{5}$ (except $\phi(v)$ ).

If $\phi(w)$ is joined with $x_{3}$, then the subdiagram $\langle\Sigma \backslash \phi(v)\rangle$ is superhyperbolic. If $\phi(w)$ is joined with $x_{5}$, then $\phi\left(N_{3} \cup\{w\}\right)$ does not belong to any Lannér subdiagram, contrary to the definition of lifting. Hence $\phi(w)$ is joined with $x_{4}$. By assumption, $\Sigma$ does not contain Lannér subdiagrams of order 3, therefore the diagram $\langle\phi(v), \phi(w)\rangle$ is Lannér, and $\Sigma=\Sigma\left(P_{8}\right)$.

Lemma 3.9. The diagram $D=\lfloor 1,3,2,3\rfloor_{2}$ has no 0-liftings.

Proof. Suppose that $\Sigma$ is a 0 -lifting of $\lfloor 1,3,2,3\rfloor_{2}$. Comparing the 0-liftings of $\lfloor 3,2,3\rfloor_{2}$ (see [8, Lemma 5.12]) with the 0-liftings of $[2,3,1\rfloor_{2}$ (see [17, Table 4.8]), we conclude that $\Sigma$ is one of the following diagrams:

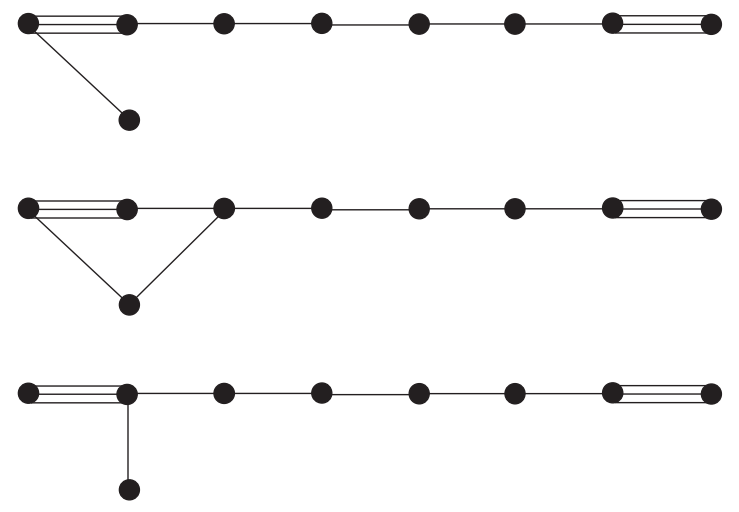

These diagrams are superhyperbolic, which proves the lemma.

Lemma 3.10. Let $D=(3,2,3,1,1)_{2}$. Then there are no 0 - or 1-liftings of $D$ of positive inertia index smaller than or equal to 8 and not containing Lannér subdiagrams of order 3.

Proof. Suppose there is such a lifting $\Sigma$ and let $\phi$ be a lifting injection. Denote the subsets of $D$ as follows: $(3,2,3,1,1)_{2}=\left(N_{1}, N_{2}, N_{3}, v, u\right)$. Set $X=\left\langle\phi\left(N_{1}\right), \phi\left(N_{2}\right), \phi\left(N_{3}\right)\right\rangle$. 
The subdiagram $X$ of $\langle\phi(D)\rangle$ does not contain small Lannér subdiagrams. Hence $X \approx$ $\lfloor 3,2,3\rfloor_{2}$, and by [8, Lemma 5.12], we have

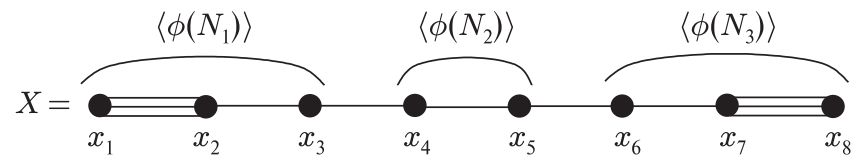

By the definition of lifting, the diagram $\left\langle\phi\left(N_{1}\right), \phi(u)\right\rangle$ belongs to some Lannér subdiagram of $\Sigma$. If $\left\langle\phi\left(N_{1}\right), \phi(u)\right\rangle$ is Lannér, then

$$
\langle X, \phi(u)\rangle \approx\left\lfloor\phi(u), \phi\left(N_{1}\right), \phi\left(N_{2}\right), \phi\left(N_{3}\right)\right\rfloor_{2}=\lfloor 1,3,2,3\rfloor_{2},
$$

which is impossible by Lemma 3.9.

Hence $\left\langle\phi\left(N_{1}\right), \phi(u)\right\rangle$ is elliptic and $\Sigma$ contains exactly one additional node $a$. Similarly, $\left\langle\phi\left(N_{3}\right), \phi(v)\right\rangle$ is also elliptic. Since $\Sigma$ does not contain Lannér subdiagrams of order $3,\langle\phi(u), \phi(v)\rangle$ is a Lannér diagram. The remaining small missing faces are $\left\langle\phi\left(N_{1}\right), \phi(u)\right\rangle$ and $\left\langle\phi\left(N_{3}\right), \phi(v)\right\rangle$. Hence each additional subdiagram of $\Sigma$ contains either $\left\langle\phi\left(N_{1}\right), \phi(u)\right\rangle$ or $\left\langle\phi\left(N_{3}\right), \phi(v)\right\rangle$, and the diagram $Y=\langle X, a, \phi(u)\rangle$ satisfies the assumptions of Lemma 2.1. Since $|Y|=10,|Y|$ is one of the diagrams $\Theta_{1}, \Theta_{2}$, and $\Theta_{3}$ of Figure 1. The diagram $\Theta_{1}$ does not contain $\Sigma_{0} \approx\lfloor 3,2,3\rfloor_{2}$. The diagrams $\Theta_{2}$ and $\Theta_{3}$ have positive inertia index 9 , contrary to the assumption.

Lemma 3.11. The diagrams $\lfloor 5,3\rfloor_{1}$ and $\lfloor 5,3,2\rfloor_{1}$ have neither 0 - nor 1-liftings.

Proof. We shall prove the lemma for $D=\lfloor 5,3\rfloor_{1}$; the assertion for $\lfloor 5,3,2\rfloor_{1}$ will then immediately follow.

By Proposition 3.1 $D$ has no 0 -liftings. Suppose that $\Sigma$ is a 1-lifting of $D, \phi$ is a lifting injection, and $a$ is the additional node. Let $M_{5}$ and $M_{3}$ be the missing faces of $D$ of order 5 and 3, respectively. Then $\left\langle\phi\left(M_{3}\right), a\right\rangle$ is a Lannér diagram. By Proposition 3.1, $\Sigma$ has at least one additional Lannér subdiagram. By the definition of lifting, any additional Lannér subdiagram of $\Sigma$ contains $\phi\left(M_{3}\right)$.

Suppose that $L=\left\langle\phi\left(M_{3}\right), x\right\rangle$ is an additional Lannér subdiagram of $\Sigma$ of order 4 , $x \in \phi\left(M_{5}\right)$. Then $x$ is an open node of $L$, since otherwise $\Sigma$ would contain a conflicting subdiagram. It is not difficult to see that $x$ is a doubly open node of $L$, whence $L=\mathcal{L}_{5}^{4}$. On the other hand, $\langle L, a\rangle \approx\left\lfloor x, M_{3}, a\right\rfloor_{2}=\lfloor 1,3,1\rfloor_{2}$. By [17, Table 4.8], no diagram $\Sigma_{0} \approx\lfloor 1,3,1\rfloor_{2}$ contains subdiagrams $\mathcal{L}_{5}^{4}$. It follows that $\Sigma$ has no additional Lannér subdiagrams of order 4 .

Thus $\Sigma$ has at least one additional Lannér subdiagram $L$ of order $5, L=\left\langle\phi\left(M_{3}\right)\right.$, $\left.x_{1}, x_{2}\right\rangle, x_{1}, x_{2} \in \phi\left(M_{5}\right)$. Since $L$ is connected, we may assume that $x_{1}$ is joined with some node $y \in \phi\left(M_{3}\right)$ (up to a transposition of $x_{1}$ and $x_{2}$ ). Then $x_{1}$ is an open node of $\left\langle\phi\left(M_{5}\right)\right\rangle$ and $y$ is an open node of $\left\langle\phi\left(M_{3}\right), a\right\rangle$. Since no Lannér diagram of order 5 has more than one open node, $x_{2}$ cannot be joined with $\left\langle\phi\left(M_{3}\right)\right\rangle$. Hence $x_{2}$ is joined with $x_{1}$. For the same reason, any additional Lannér subdiagram $L^{\prime}$ contains $x_{1}$; moreover, if $x_{k} \in L^{\prime}$ and $x_{k} \neq x_{1}$, then $x_{k}$ is joined with $x_{1}$ and is not joined with $\left\langle\phi\left(M_{3}\right)\right\rangle$.

If $L$ is the only additional Lannér subdiagram of $\Sigma$, then

$$
\Sigma \approx\left\lfloor\left\langle\phi\left(M_{5}\right) \backslash\left\{x_{1}, x_{2}\right\}\right\rangle,\left\langle x_{1}, x_{2}\right\rangle,\left\langle\phi\left(M_{3}\right)\right\rangle, a\right\rfloor_{2}=\lfloor 3,2,3,1\rfloor_{2},
$$

which is impossible by Lemma 3.9. Therefore, $\Sigma$ contains at least one more additional Lannér subdiagram $L^{\prime}$. Suppose that $L^{\prime}=\left\langle\phi\left(M_{3}\right), x_{1}, x_{3}\right\rangle, x_{3} \in \phi\left(M_{5}\right), x_{3} \neq x_{2}$. As was shown above, the open node $x_{1}$ of $\left\langle\phi\left(M_{5}\right)\right\rangle$ is joined with two other nodes, $x_{2}$ and $x_{3}$, which implies that $\left\langle\phi\left(M_{5}\right)\right\rangle=\mathcal{L}_{5}^{5}$. In particular, the edges $x_{1} x_{2}$ and $x_{1} x_{3}$ are simple. 
Keeping in mind that

$$
\langle L, a\rangle \approx\left\lfloor a,\left\langle\phi\left(M_{3}\right)\right\rangle,\left\langle x_{1}, x_{2}\right\rangle\right\rfloor_{2}=\lfloor 1,3,2\rfloor_{2}
$$

and

$$
\left\langle L^{\prime}, a\right\rangle \approx\left\lfloor a,\left\langle\phi\left(M_{3}\right)\right\rangle,\left\langle x_{1}, x_{3}\right\rangle\right\rfloor_{2}=\lfloor 1,3,2\rfloor_{2},
$$

we check all possibilities for $\lfloor 1,3,2\rfloor_{2}$ such that $x_{1} x_{2}$ and $x_{1} x_{3}$ are simple edges (see [17, Table 4.8]). We then have that either $\Sigma$ contains a conflicting subdiagram or $\Sigma$ is one of the following diagrams:
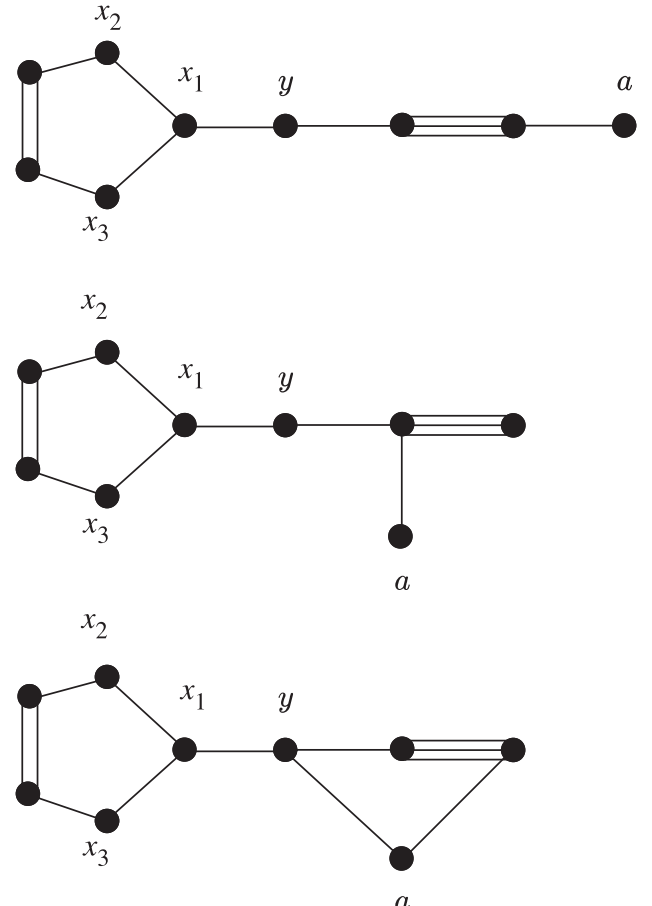

All these diagrams are superhyperbolic, which proves the lemma.

\section{Non-existence of Polytopes in Dimensions $\geq 9$}

Suppose that there is a compact hyperbolic Coxeter $d$-polytope $P$ with $d+4$ facets. Let $\Sigma$ be the Coxeter diagram of $P$.

For the study of polytopes with $d+4$ facets we shall refer to the classification of compact Coxeter $d$-polytopes with $d+1, d+2$, and $d+3$ facets (see [14, 9, 13, 17). In particular, recall that $P_{8}$ is the only 8-polytope with 11 facets, and $\Sigma\left(P_{8}\right)$ stands for its Coxeter diagram (see Figure 3).

Henceforth, by a polytope we understand a compact hyperbolic Coxeter polytope, and by the diagram of a polytope, the Coxeter diagram of it.

Lemma 4.1. Suppose that $d \geq 9$. Then:

(1) any node $v$ of $\Sigma$ is incident to at most one non-simple edge;

(2) $\Sigma$ has no Lannér subdiagrams of order 3;

(3) $\Sigma$ has no edges of multiplicity $\geq 4$.

Proof. If the lemma is not true, then $\Sigma$ has either a node incident to two dashed edges or a subdiagram $S_{0} \subset \Sigma$ of type $G_{2}^{(m)}, m \geq 4$, with a bad neighbor. 
Suppose that $\Sigma$ has a node $v$ incident to two dashed edges. Then the facet $f=P(v)$ is a simple (possibly non-Coxeter) $(d-1)$-polytope with at most $d+1=(d-1)+2$ facets, i.e., either a simplex or a product of two simplices. If $f$ is a simplex, then $P$ has a large missing face, contrary to Corollary 3.1. If $f$ is a product of two simplices and has no large missing faces, then it is a product of two 4-simplices (since $d \geq 9$ ), and therefore $D(f)=\lfloor 5,5\rfloor_{1}$. By Lemma 3.3 and Proposition 3.1, the diagram $\lfloor 5,5\rfloor_{1}$ has no liftings, contrary to Lemma 3.2 .

Suppose that $\Sigma$ has a subdiagram $S_{0} \subset \Sigma$ of type $G_{2}^{(m)}$ with $m \geq 4$ and a bad neighbor. Then $P\left(S_{0}\right)$ is a Coxeter $(d-2)$-polytope with at most $(d-2)+3$ facets (see Proposition 1.11), therefore either $d-2=8$ or $d-2 \leq 6$ (see [17). If $d-2 \leq 6$, then $d \leq 8$, contrary to the assumption. If $d-2=8$, then $d=10$ and $P\left(S_{0}\right)$ is the only Coxeter 8-polytope with 11 facets (see Figure 3). Notice that $\Sigma_{S_{0}}$ has a subdiagram of type $H_{4}$ with two neighbors joined with it by simple edges. In view of Corollary 1.2, the corresponding diagram $S_{1} \subset \Sigma$ is also of type $H_{4}$ with at least two neighbors. Hence $P\left(S_{1}\right)$ is a Coxeter 6-polytope with at most eight facets, which is impossible (see [9, 13]).

a)

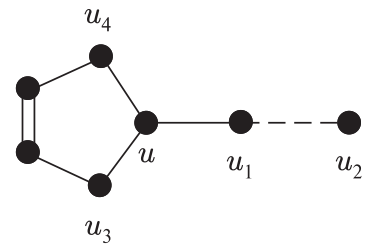

b)

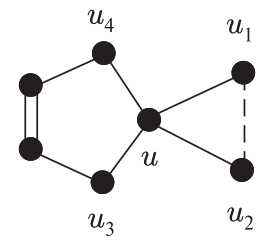

c)
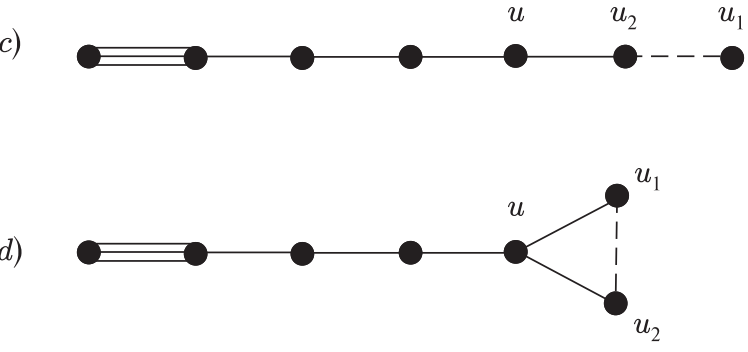

FiguRE 4. The 5-prisms that have property (1) in Lemma 4.1

Lemma 4.2. Suppose $d \geq 9$. Then $\Sigma$ does not contain Lannér subdiagrams of order 5 .

Proof. Suppose that there is a Lannér subdiagram $L_{0} \subset \Sigma$ of order 5 . The classification of Lannér diagrams shows that there is a subdiagram $S_{0} \subset L_{0}$ of type $H_{4}$ or $F_{4}$. Then $P\left(S_{0}\right)$ is a Coxeter $(d-4)$-polytope and $\Sigma_{S_{0}}=\bar{S}_{0}$ is a subdiagram of $\Sigma$ (see Corollary 1.1). Since $S_{0}$ has at least one neighbor (the node $L_{0} \backslash S_{0}$ ) and a neighbor of a diagram of type $H_{4}$ or $F_{4}$ cannot be good, the order of $\Sigma_{S_{0}}$ is either $d-3$, or $d-2$, or $d-1$. 
Case 1. $P\left(S_{0}\right)$ has $d-3$ facets. Then $P\left(S_{0}\right)$ is a Coxeter simplex and $d-4 \leq 4$, i.e., $d \leq 8$, contrary to the assumption.

Case 2. $P\left(S_{0}\right)$ has $d-2$ facets, i.e., $P\left(S_{0}\right)$ is a Coxeter $(d-4)$-polytope with $(d-4)+2$ facets, and therefore (see [8, 13) $d-4 \leq 5$, i.e., $d \leq 9$. Hence $d=9$ and $P\left(S_{0}\right)$ is a 5-prism. Since $\Sigma_{S_{0}}=\bar{S}_{0}$, Lemma 4.1(1) together with 13 implies that $\bar{S}_{0}$ is one of the four diagrams of Figure 4 .

Let $L_{1}$ be the only Lannér subdiagram of $\bar{S}_{0}, S_{1} \subset L_{1}$ the only subdiagram of type $H_{4}$ or $F_{4}$, and $u=L_{1} \backslash S_{1}$. The diagram $\Sigma$ consists of the following parts:

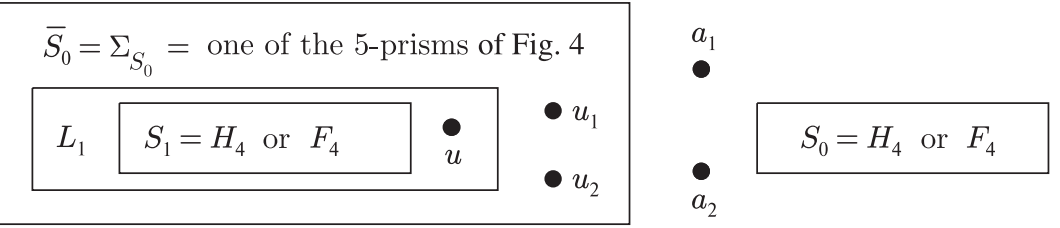

where $a_{1}$ and $a_{2}$ are neighbors of $S_{0}$, and $S_{0}$ is not joined with $\bar{S}_{0}$. Since any two indefinite subdiagrams must be joined in $\Sigma$, each of the nodes $a_{1}$ and $a_{2}$ is joined with $L_{1}$. If each of these nodes is joined with $S_{1}$, then $S_{1}$ has three neighbors, which is impossible (see Case 1). Hence one of these nodes, say $a_{1}$, is joined with $u=L_{1} \backslash S_{1}$ (see Figure 4) and is not joined with $S_{1}$.

Consider diagrams $a$ ) and $b$ ) in Figure 4 with the nodes indexed as is shown there. Let $S_{2}=\Sigma_{S_{0}} \backslash\left\langle u_{2}, u_{4}\right\rangle$. Then $S_{2}$ is of type $B_{5}$ with at least three bad neighbors $\left(u_{4}\right.$, $u_{2}$, and $a_{1}$ ). By Lemma 1.3, $a_{2}$ cannot be a neighbor of $S_{2}$. Similarly, examining $S_{3}=\Sigma_{S_{0}} \backslash\left\langle u_{2}, u_{3}\right\rangle$ we have that $a_{2}$ is not joined with $u_{4}$. Hence $a_{2}$ is not joined with $L_{1}$, i.e., the indefinite subdiagram $\left\langle S_{0}, a_{2}\right\rangle$ is not joined with the Lannér diagram $L_{1}$, which is impossible.

Suppose now that $\Sigma_{S_{0}}$ is one of the diagrams $c$ ) and $d$ ) in Figure 4. There are two possibilities: either $a_{2}$ is joined with $S_{1}$ or not. Suppose $a_{2}$ is not joined with $S_{1}$. Then $P\left(S_{1}\right)$ is a Coxeter 5-polytope with eight facets. By Corollary 1.1 $\Sigma_{S_{1}}=\bar{S}_{1} \subset \Sigma$. But property (1) in Lemma 4.1 is not shared by any Coxeter 5-polytope with eight facets (see [17). Hence $a_{2}$ is joined with $S_{1}$, and $S_{1}$ has exactly two neighbors, $u$ and $a_{2}$ (it cannot have more neighbors by Case 1). Thus, $P\left(S_{1}\right)$ is a Coxeter 5-prism. We may assume that the diagram $\Sigma_{S_{1}}$ of $P\left(S_{1}\right)$ is of type $c$ ) or $d$ ) in Figure 4 (in the case of prisms $a$ ) and $b$ ) use the arguments from the previous paragraph). Notice that $\Sigma_{S_{1}}=\bar{S}_{1}$ contains $S_{0}$; hence $S_{0}$ is a diagram of type $H_{4}$.

Furthermore, $\Sigma_{S_{1}}$ contains $u_{1}, u_{2}$, and $a_{1}$. Since $P\left(S_{1}\right)$ is a 5 -prism, $\Sigma_{S_{1}}$ contains a Lannér subdiagram of order 5 . On the other hand, $\Sigma_{S_{1}}=\left\langle S_{0}, u_{1}, u_{2}, a_{1}\right\rangle$, where $u_{1}$ and $u_{2}$ are not joined with $S_{0}$, and therefore $\left\langle S_{0}, a_{1}\right\rangle$ is a Lannér diagram. Hence $\left\langle S_{0}, a_{1}\right\rangle$ is joined with the Lannér diagram $L_{1}$, i.e., $a_{1}$ is joined with $u$. By Lemma 1.4, $a_{1}$ is joined with $u$ by a dashed edge. Thus, $\left\langle S_{0}, a_{1}, u, S_{1}\right\rangle$ is of the form

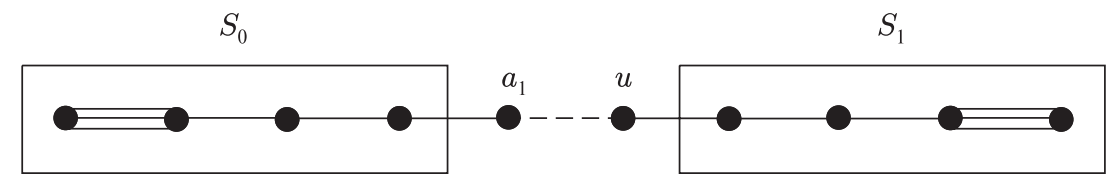


Recall that $u_{1}$ and $u_{2}$ are joined by a dashed edge. Since $u_{1}, u_{2} \in \bar{S}_{0}$, each of these nodes is joined in $\Sigma \backslash a_{2}$ with $u$ and $a_{1}$ only. For $\Sigma \backslash a_{2}$ we have four possibilities:
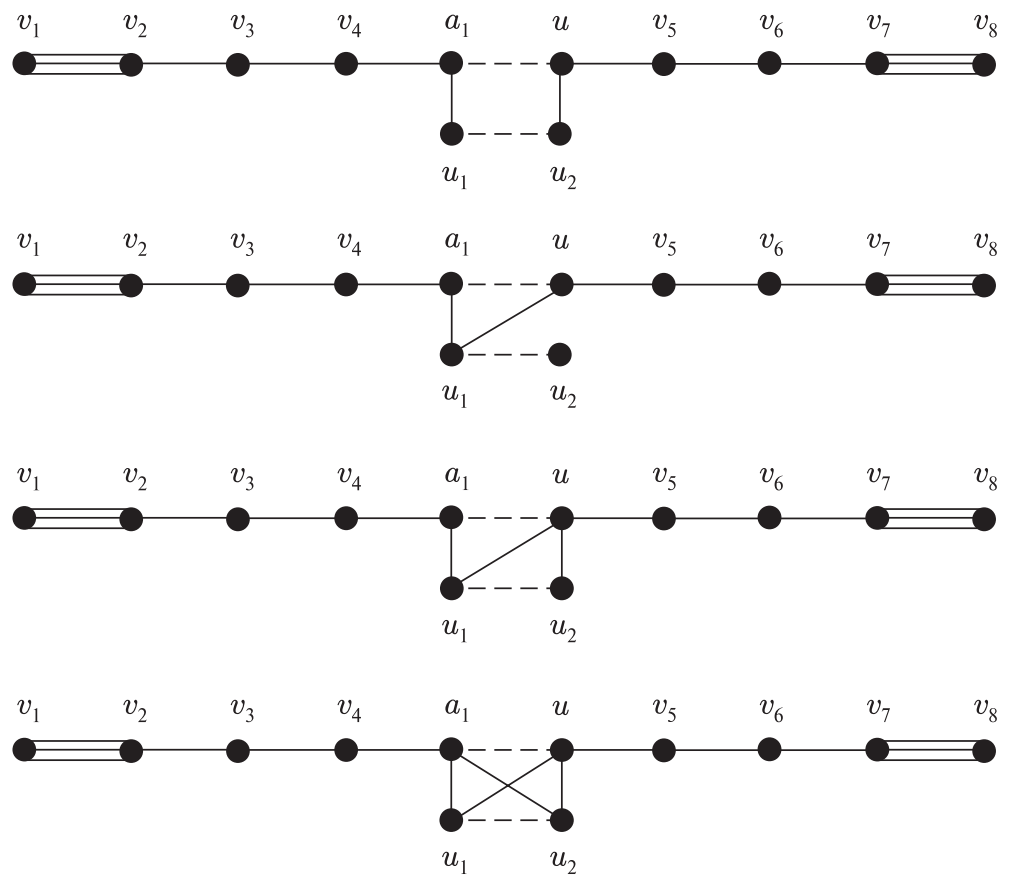

By assumption, $a_{2}$ is joined with $S_{1}=\left\langle v_{5}, v_{6}, v_{7}, v_{8}\right\rangle$. Since $a_{2} \notin \Sigma_{S_{0}}, a_{2}$ is joined with $S_{0}=\left\langle v_{1}, v_{2}, v_{3}, v_{4}\right\rangle$. Furthermore, $a_{2}$ is joined with $\left\langle u_{1}, u_{2}\right\rangle$, since otherwise the indefinite diagram $\left\langle S_{0}, a_{2}\right\rangle$ would not be joined with the Lannér diagram $\left\langle u_{1}, u_{2}\right\rangle$. Since each of the diagrams $\left\langle v_{2}, v_{3}, v_{4}, a_{1}, u_{1}\right\rangle,\left\langle v_{7}, v_{6}, v_{5}, u, u_{1}\right\rangle$, and $\left\langle v_{7}, v_{6}, v_{5}, u, u_{2}\right\rangle$ has three bad neighbors, no dashed edge ends in $a_{2}$ (see Lemma 4.1 (1)). Examining the possible multiplicities of the edges (i.e., simple, double, triple, and empty), we see that there is always either a Lannér subdiagram of order 3, or a parabolic subdiagram, or a subdiagram of type $\mathrm{H}_{4}$ with at least three neighbors (the latter is in fact impossible; see Case 1).

Case 3. $P\left(S_{0}\right)$ has $d-1$ facets. Then $P\left(S_{0}\right)$ is a Coxeter $(d-4)$-polytope with $(d-4)+3$ facets. Since $d \geq 9$, we have $d-4 \geq 5$. By [17], the Coxeter diagrams of such polytopes either do not have properties (1)-(3) of Lemma 4.1 or have a subdiagram of type $H_{4}$ with at least two neighbors, which is impossible by the previous cases.

Lemma 4.3. Suppose that one of the following holds:

1) $d \geq 9$;

2) $d=8, \Sigma$ has properties (1)-(3) of Lemma 4.1, and $\Sigma$ does not contain Lannér subdiagrams of order 5 .

Then $\Sigma$ has no Lannér subdiagrams of order 4 .

Proof. Suppose that $L_{0}$ is a Lannér subdiagram of $\Sigma$ of order 4 . Let $S_{0} \subset L_{0}$ be a subdiagram of type $H_{3}$ (if any) or $B_{3}$ (otherwise). Then $P\left(S_{0}\right)$ is a Coxeter $(d-3)$ polytope with at most $(d-3)+3$ facets. By Lemmas 4.2 and $3.2 \Sigma_{S_{0}}$ has no Lannér subdiagrams of order 5 . If $P\left(S_{0}\right)$ is a simplex or a product of two simplices, then $d-3 \leq 3$ or $d-3 \leq 4$, respectively; hence $d \leq 7$, contrary to the assumption.

Suppose that $P\left(S_{0}\right)$ is a polytope with $(d-3)+3$ facets. When $d \geq 8$, the Coxeter diagram of almost all $(d-3)$-polytopes with $d$ facets contains a Lannér subdiagram of 
order 5 (see [17]). The only exception is a 5-polytope with eight facets and Coxeter diagram $\Sigma_{S_{0}}$ of the form

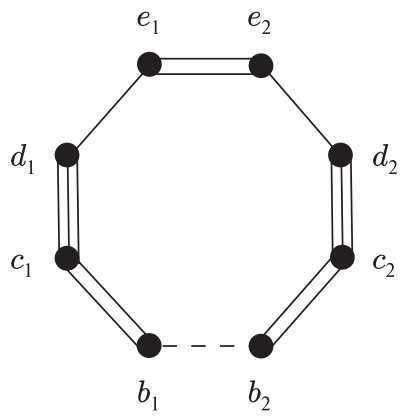

By Corollary 1.2 no node of $\bar{S}_{0} \backslash\left\langle b_{1}, b_{2}\right\rangle$ is a good neighbor of $S_{0}$. The node $b_{1}$ is a good neighbor; otherwise $\Sigma$ would contain the Lannér subdiagram $\left\langle b_{1}, c_{1}, d_{1}\right\rangle$ of order 3 , contrary to the assumption. Thus, $\left\langle b_{1}, c_{1}\right\rangle$ is a simple edge of $\Sigma$ and $\left\langle d_{1}, c_{1}, b_{1}, x_{1}, x_{2}\right\rangle$ is a Lannér diagram of order 5 (here $x_{1}$ and $x_{2}$ are the ends of the simple edge of $S_{0}$ ). This contradicts the assumption.

Theorem 1. There are no compact hyperbolic Coxeter $d$-polytopes with $d+4$ facets with $d \geq 9$.

Proof. It follows from Lemmas 4.1, 4.2, and 4.3 that the order of any Lannér subdiagram of $\Sigma$ is 2. It was shown in [8, Prop. 6.9] that such a polytope with $d+4$ facets may only exist when $d \leq 4$.

\section{NON-EXISTENCE OF POLYTOPES IN DIMENSION 8}

In this section we show that there are no compact Coxeter 8-polytopes with 12 facets. Assuming that $P$ is such a polytope and $\Sigma$ is its Coxeter diagram, we show that the properties of $\Sigma$ are similar to those proved for polytopes in large dimensions, which eventually leads to a contradiction. However, the proofs in the 8-dimensional case are much more complicated than in larger dimensions.

Lemma 5.1 ([11, Lemma 1]). Let $\Sigma(P)$ be the Coxeter diagram of a Coxeter d-polytope $P$. Then no proper subdiagram of $\Sigma(P)$ is the diagram of a Coxeter d-polytope of finite volume.

In particular, $\Sigma$ does not contain $\Sigma\left(P_{8}\right)$ as a proper subdiagram.

Lemma 5.2. Suppose $\left\langle v_{1}, v_{2}\right\rangle \subset \Sigma$ is a subdiagram of type $G_{2}^{(k)}, k \geq 4$. Then:

(1) $\left\langle v_{1}, v_{2}\right\rangle$ has at most one bad neighbor;

(2) $k \leq 5$

(3) if $\left\langle v_{1}, v_{2}\right\rangle$ has a bad neighbor, then it also has a good neighbor.

Proof. Let $S_{0}=\left\langle v_{1}, v_{2}\right\rangle$. If $S_{0}$ has two bad neighbors, then $P\left(S_{0}\right)$ is a Coxeter 6-polytope with at most eight facets, which is impossible by [13, 8 .

Suppose that either $k \geq 6$ or $S_{0}$ has a bad neighbor and no good ones. In particular, all neighbors of $S_{0}$ are bad and, by Corollary 1.1, $\bar{S}_{0}=\Sigma_{S_{0}}$. In addition, $P\left(S_{0}\right)$ is a Coxeter 6-polytope with at most nine facets. By [17, the diagram of such a polytope is one of the three diagrams of Figure 5. The Coxeter diagram of each of those polytopes contains a multiple edge with two bad neighbors. This is impossible, as we have shown before. 
Lemma 5.3. (1) $\Sigma$ does not contain Lannér subdiagrams of order 3.

(2) No node incident to a dashed edge is incident to a triple edge.

(3) If $\Sigma$ has exactly one dashed edge, then neither of its ends is incident to a double edge.

Proof. Suppose that the lemma is not true. Let $L_{0}$ be a Lannér subdiagram of order 3 or a subdiagram with three nodes that contains a dashed edge and a multiple edge. Consider two cases.

Case $1 . L_{0}$ contains a triple edge.

Let $S_{0}$ be the subdiagram consisting of that edge and its ends. Then $P\left(S_{0}\right)$ is a Coxeter 6-polytope with at most nine facets (since $L_{0}$ contains a bad neighbor of $S_{0}$ ). Then $\Sigma_{S_{0}}$ is one of the three diagrams of Figure 5. By Corollary 1.1, $\bar{S}_{0} \subset \Sigma$ can be obtained from $\Sigma_{S_{0}}$ by replacing (if necessary) some dashed edges with ordinary edges and some edges labeled by 10, with simple edges. Applying this procedure to $P_{6}^{1}$ and $P_{6}^{3}$ (Figure 51), we see that the resulting diagrams contain either a parabolic subdiagram or a multiple edge with two bad neighbors.

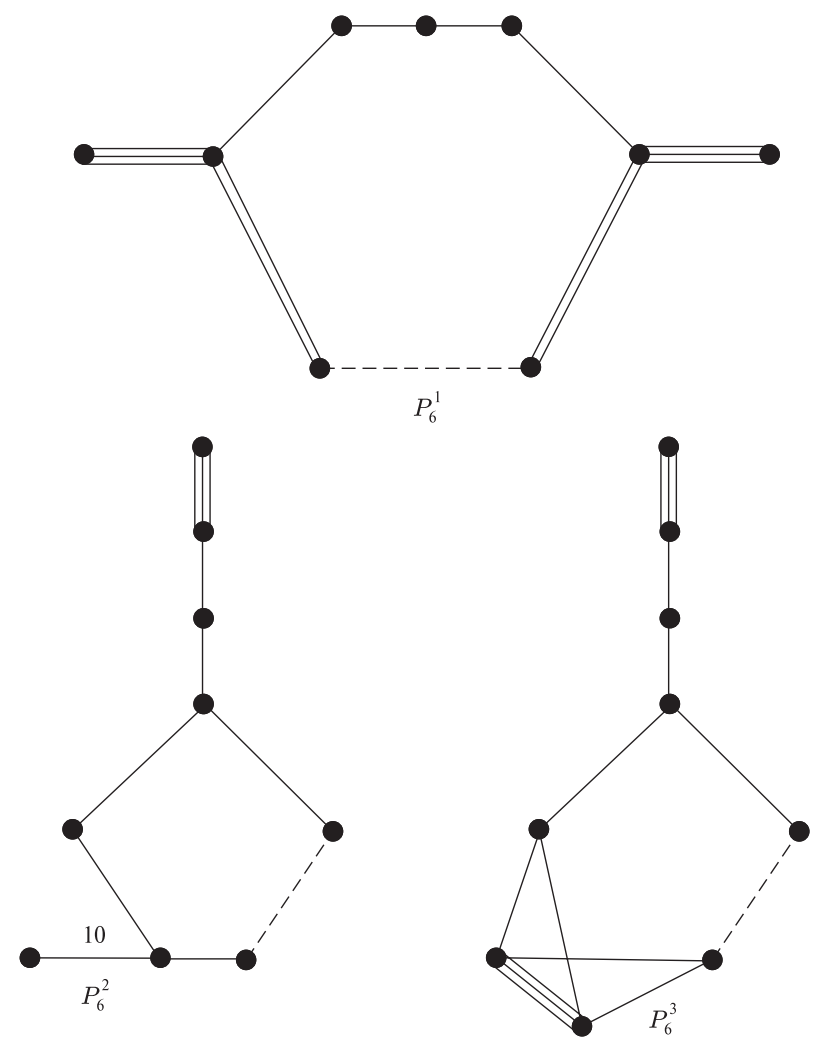

Figure 5. Compact hyperbolic Coxeter 6-polytopes with nine facets

Suppose that $P\left(S_{0}\right)=P_{6}^{2}$. Let $u$ be the bad neighbor of $S_{0}$ (it is unique by Lemma 5.2). In view of Corollary 1.1, $\bar{S}_{0}$ can be obtained from $\Sigma_{S_{0}}$ by replacing some edges labeled by 10 with simple edges and some dashed edges with ordinary ones. By Corollary 1.2. the dashed edge of $\Sigma_{S_{0}}$ remains dashed in $\bar{S}_{0}$. By Lemma [5.2, the edge of $\Sigma_{S_{0}}$ labeled by 10 is a simple edge in $\bar{S}_{0}$. Hence the leaf of $\Sigma_{S_{0}}$ incident to the edge labeled by 10 is a good neighbor of $S_{0}$ in $\Sigma$. The remaining nodes of $\Sigma_{S_{0}}$ cannot be good neighbors of 
$S_{0}$ by Corollary 1.2. Thus, $\Sigma \backslash u$ is the Coxeter diagram $\Sigma\left(P_{8}\right)$, which is impossible by Lemma 5.1 .

Case 2. $L_{0}$ has no triple edges.

Let $S_{0}$ be the subdiagram of $L_{0}$ consisting of a double edge and its ends. As before, $P\left(S_{0}\right)$ is a 6-polytope with nine facets. By Corollary $1.2, \bar{S}_{0} \subset \Sigma$ can be obtained from $\Sigma_{S_{0}}$ by replacing (if necessary) some dashed edges with ordinary ones and some double edges, with simple or empty ones. Applying this procedure to the diagrams of the polytopes $P_{6}^{3}$ and $P_{6}^{2}$, we conclude that the resulting diagrams contain multiple edges with at least two bad neighbors, which is impossible by Lemma 5.2 .

Suppose that $P\left(S_{0}\right)=P_{6}^{1}$. Then both double edges of $\Sigma_{S_{0}}$ become simple or empty in $\bar{S}_{0}$; otherwise $\Sigma$ would have a Lannér subdiagram with a triple edge, which is impossible by Case 1. Moreover, by Corollary [1.2, only one end of each double edge can be a good neighbor of $S_{0}$. Therefore, by Proposition 1.11 each double edge of $\Sigma_{S_{0}}$ is simple in $\bar{S}_{0}$. Moreover, the only dashed edge remains dashed, since otherwise $\bar{S}_{0}$ would be superhyperbolic. Hence in this case we may assume that $L_{0}$ has no dashed edges (otherwise all assertions of the lemma are obviously true). By Proposition 1.11, the ends of the dashed edge of $\bar{S}_{0}$ are good neighbors of $S_{0}$, whereas the remaining nodes of $\bar{S}_{0}$ are not. Consider the subdiagram $S_{1} \subset \bar{S}_{0}$ of type $H_{4}$ consisting of a triple edge, a simple edge adjacent to it and obtained from a double edge, and a simple edge joining $S_{0}$ with its good neighbor. The diagram $S_{1}$ has two neighbors in $\bar{S}_{0}$ and two neighbors in $S_{0}$ (since $L_{0}$ is a Lannér diagram with no edges of multiplicity greater than 2 ). This means that $S_{1}$ has at least four bad neighbors, contrary to Lemma 1.3 .

The next lemma is the main result of [11.

Lemma 5.4 ([11, Th. A]). Let $P$ be a compact Coxeter polytope in the d-dimensional hyperbolic space, and $\Sigma(P)$ its Coxeter diagram. If $d>4$, then $\Sigma(P)$ contains a dashed edge.

Lemma 5.5. Let $S \subset \Sigma$ be an elliptic subdiagram of order 3 having no components of type $A_{n}$. Then $S$ has at most two bad neighbors.

Proof. Suppose that $S$ has three or more bad neighbors. Then $\Sigma_{S}$ is a 5-polytope with at most six facets, which is impossible.

Lemma 5.6. Suppose that $\Sigma$ contains a Lannér subdiagram $L_{0}$ of order 5 and a unique dashed edge. Then $\Sigma$ contains a subdiagram $S$ of type $F_{4}$ or $H_{4}$ such that $P(S)$ is not a simplex.

Proof. Suppose that the lemma is not true, i.e., for any diagram $S^{\prime} \subset \Sigma$ of type $H_{4}$ or $F_{4}$ the face $P\left(S^{\prime}\right)$ is a simplex.

Let $S_{0} \subset L_{0}$ be a diagram of type $H_{4}$ or $F_{4}$. Then $P\left(S_{0}\right)$ is a Coxeter 4 -simplex. The diagram $\Sigma_{S_{0}}$ is a Lannér diagram of order 5 , we denote it $L_{1}$. Let $S_{1}$ be a subdiagram of $L_{1}$ of type $H_{4}$ or $F_{4}$. By assumption, $P\left(S_{1}\right)$ is a Coxeter simplex, and $\bar{S}_{1}=\Sigma_{S_{1}}$ is a Lannér diagram of order 5 , we denote it $L_{2}$. Notice that $S_{0} \subset L_{2}$ and let $S_{2}=S_{0}$. Let $v_{i}=L_{i} \backslash S_{i}, i=0,1,2$.

Since $S_{2}=S_{0}$ is not joined with $L_{1}, v_{2}$ is joined with $L_{1}$. On the other hand, $L_{2}$ is not joined with $S_{1}$. Hence $\left\langle L_{1}, L_{2}\right\rangle$ consists of two Lannér subdiagrams $L_{1}$ and $L_{2}$ joined by a single edge $v_{1} v_{2}$ such that $L_{i} \backslash v_{i}$ is of type $H_{4}$ or $F_{4}$. By Lemma 1.4 $v_{1} v_{2}$ is a dashed edge.

Let $a$ and $b$ be nodes not contained in $\left\langle L_{1}, L_{2}\right\rangle$. Notice that if $v_{2} \neq v_{0}$, then either $a$ or $b$ coincides with $v_{0}$. Both $a$ and $b$ are joined with $S_{1}$ and $S_{2}$. The diagram $\Sigma$ consists 
of the following parts:

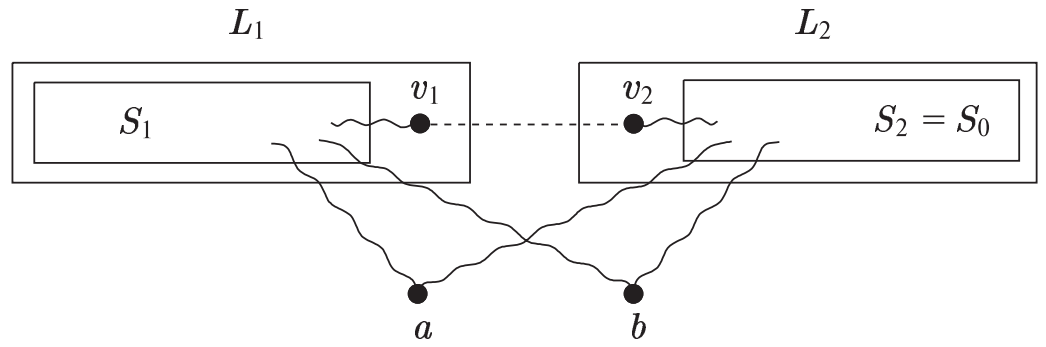

where the wavy line indicates that the node is joined with the subdiagram by a nondashed edge; the subdiagram $\left\langle a, b, v_{1}, v_{2}\right\rangle$ may contain some other (non-dashed) edges.

By Lemma 5.3. $v_{1}$ and $v_{2}$ are joined with $S_{1}$ and $S_{2}$ only by simple edges. Hence $L_{1}$ (as well as $L_{2}$ ) is one of the diagrams $\mathcal{L}_{1}^{5}, \mathcal{L}_{4}^{5}$, or $\mathcal{L}_{5}^{5}$ from Table 2 .

Suppose that $L_{1}=\mathcal{L}_{5}^{5}$. Let $w_{1} \in S_{1}$ be a node joined with $a$. If $w_{1} a$ is a triple edge, then $\Sigma$ has either a Lannér subdiagram of order 3 or a diagram of type $H_{3}$ with at least three bad neighbors, which is impossible by Corollary 3.1. If $w_{1} a$ is a double or a simple edge, then we have either a parabolic subdiagram or a Lannér subdiagram of order 3 . Thus the multiplicity of $w_{1} a$ should be greater than three, which is impossible by Lemma 5.2 .

Hence both $S_{1}$ and $S_{2}$ are of type $H_{4}$. For each of the four possible pairs of diagrams $L_{1}$ and $L_{2}$, there is a unique label for the dashed edge $v_{1} v_{2}$ such that the determinant of $\Sigma \backslash\langle a, b\rangle$ vanishes. Those labels are: $(1+\sqrt{5}) / 2$ for $L_{1}=L_{2}=\mathcal{L}_{1}^{5}, 4+2 \sqrt{5}$ for $L_{1}=L_{2}=\mathcal{L}_{4}^{5}$, and $(3+\sqrt{5}) / \sqrt{2}$ for $L_{1}=\mathcal{L}_{1}^{5}, L_{2}=\mathcal{L}_{4}^{5}\left(\right.$ or $\left.L_{2}=\mathcal{L}_{1}^{5}, L_{1}=\mathcal{L}_{4}^{5}\right)$.

Let $u_{1} \in S_{1}$ and $u_{2} \in S_{2}$ be the leaves of $\Sigma \backslash\langle a, b\rangle$ incident to the triple edges. Suppose that both $a$ and $b$ are joined with $S_{1} \backslash u_{1}$ and $S_{2} \backslash u_{2}$, all edges joining $a$ and $b$ with $S_{i} \backslash u_{i}$ are simple, and $a$ is not joined with $b$. Then $\Sigma \backslash\left\langle v_{1}, v_{2}\right\rangle$ contains a parabolic subdiagram of type $\widetilde{A}_{m}$ for some $m, 2 \leq m \leq 7$. If in addition there is a multiple edge joining $a$ or $b$ with $S_{1} \backslash u_{1}$ and $S_{2} \backslash u_{2}$, or $a$ and $b$ are joined, then $\Sigma \backslash v_{1}$ or $\Sigma \backslash v_{2}$ contains either a diagram of type $H_{3}$ with at least three neighbors, or a Lannér diagram of order 3 , or a parabolic diagram of type $B_{3}$. Hence at least one of the nodes $a$ and $b$, say $a$, is joined with one of the diagrams $S_{i} \backslash u_{i}$, say $S_{1} \backslash u_{1}$, by multiple edges only. Thus, by Lemma 5.3 , there are two possibilities: either $a$ is joined with $S_{1} \backslash u_{1}$ by a single multiple edge or it is not joined with $S_{1} \backslash u_{1}$ (in the latter case $a$ is joined with $u_{1}$ ).

Case 1 . The node $a$ is joined with $S_{1} \backslash u_{1}$ by a single multiple edge $a u$. It is not difficult to see that if $L_{1}=\mathcal{L}_{4}^{5}$, then $u$ is a leaf of $L_{1}$ different from $v_{1}$, and $a$ is not joined with $v_{1}$ (otherwise we would have either a diagram of type $H_{3}$ with at least three bad neighbors, or a Lannér subdiagram of order 3 , or a parabolic subdiagram). If au is a double edge, then $\left\langle a, L_{1} \backslash u_{1}\right\rangle$ is a parabolic subdiagram of type $\widetilde{B}_{4}$. Suppose that $a u$ is a triple edge. The node $a$ is not joined with $u_{1}$; otherwise $\left\langle u, a, u_{1}\right\rangle$ would be of type $H_{3}$ with at least three bad neighbors, which is impossible by Corollary 3.1. Consider now the diagram $\Sigma_{1}=\left\langle L_{1}, a, v_{2}\right\rangle$. The node $a$ can be joined only with $v_{2}$ (and only by a simple edge, according to Lemma 5.3). A computation shows that $\Sigma_{1}$ is superhyperbolic, whether or not the nodes $a$ and $v_{2}$ are joined.

Case 2. The node $a$ is not joined with $L_{1} \backslash u_{1}$. Then $a$ is joined with $u_{1}$ by a simple edge. As before, consider the diagram $\Sigma_{1}=\left\langle L_{1}, a, v_{2}\right\rangle$. The node $a$ can be joined only with $v_{1}$ and $v_{2}$ and only by simple edges. For each of the four pairs of diagrams $L_{1}$ and $L_{2}$, the diagram $\Sigma_{1}$ is superhyperbolic whether or not $a$ is joined with $v_{1}$ and $v_{2}$.

Lemma 5.7. Any node of $\Sigma$ is incident to at most one dashed edge. 
Proof. Suppose that $u \in \Sigma$ is a node incident to two or more dashed edges. By Lemma 1.3. if $u$ is incident to more than two dashed edges, then $P(u)$ is a 7-face of a Coxeter polytope with at most eight facets, which is impossible by Corollary 3.1 . Thus, $u$ is incident to exactly two dashed edges, and $P(u)$ is a 7-polytope with nine facets, i.e., a product of two simplices whose diagram of missing faces is $\lfloor k, 9-k\rfloor_{1}(k<9)$. Since the diagram of missing faces of this polytope cannot have large missing faces, it is of the form $\lfloor 4,5\rfloor_{1}$. By Lemma 3.4, this diagram has only one lifting, $\Theta_{1}$ in Figure 1, Denote the nodes of $\Theta_{1}$ as shown in Figure [6, and let $v$ and $w$ be the remaining nodes of $\Sigma$. Each of them is joined with $u$ by a dashed edge and they are also joined with the subdiagram $\left\langle v_{5}, \ldots, v_{9}\right\rangle \subset \Sigma$.

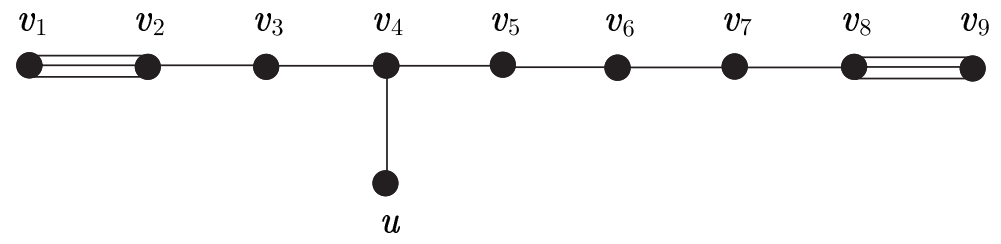

Figure 6. Unique lifting of $\lfloor 4,5\rfloor_{1}$

Case 1. The nodes $v$ and $w$ are not joined with the subdiagram $\Sigma \backslash\langle v, w, u\rangle=\left\langle v_{1}, \ldots, v_{9}\right\rangle$ by dashed edges.

Let $S_{0}=\left\langle v_{2}, \ldots, v_{8}\right\rangle$. Then $P\left(S_{0}\right)$ is a 1-face, and therefore $S_{0}$ belongs to exactly two elliptic diagrams of order 8 . This means that one of the nodes $v$ and $w$, say $v$, either is not joined with $S_{0}$ or is a good neighbor of $S_{0}$. In particular, any double edge joining $v$ with $S_{0}$ may end in $v_{2}$ or $v_{8}$ only. By Lemma 5.3 no double edge joins $v$ and $\Sigma \backslash\langle v, w, u\rangle$. Hence $v$ can be joined only with $v_{1}, v_{9}$, and one of the nodes of $S_{0}$ and only by simple edges. A straightforward calculation shows that the positive inertia index of any diagram $\Sigma \backslash\langle w, u\rangle$ thus obtained is different from 8 , contrary to the assumption that $\Sigma$ is the diagram of an 8-polytope.

Case 2. At least one of the nodes $v$ and $w$, say $v$, is joined by a dashed edge with some node $v_{x} \in \Sigma \backslash\langle v, w, u\rangle$.

In this case, $P(v)$ is a 7 -face of $P$ bounded by nine facets, i.e., a face with diagram of missing faces of type $\lfloor 5,4\rfloor_{1}$. The only lifting of this diagram is the diagram $\Theta_{1}$ (Lemma 3.4). In other words, $\Sigma \backslash\left\langle u, v_{x}\right\rangle$ looks like the diagram of Figure 6, where $v$ takes the place of $u, w$ takes the place of $v_{x}$, and, possibly, the node $v$ is not joined with $v_{4}$ but is joined with $v_{6}$ (when $v_{x} \neq v_{6}$ ) or with $w$ (when $v_{x}=v_{6}$ ).

If $v_{x}$ and $w$ are not joined by a dashed edge, we obtain a parabolic diagram $\left\langle v_{x-1}, v_{x}\right.$, $\left.v_{x+1}, w\right\rangle$ (when $v_{x} \neq v_{1}$ or $v_{9}$ ) or a Lannér diagram of order 3 (when $v_{x}=v_{1}$ or $v_{9}$ ). If $v_{x}$ and $w$ are joined by a dashed edge, consider the face $P\left(v_{x}\right)$. It is again a 7-polytope with nine facets, but in the lifting of its diagram of missing faces, the node $v_{x}$ is joined with at least two nodes, contrary to Lemma 3.2 .

Lemma 5.8. $\Sigma$ contains at least two dashed edges.

Proof. By Lemma 5.4, $\Sigma$ has a dashed edge. Suppose it is unique. Then by Lemmas 5.2 and [5.3, $\Sigma$ satisfies assertions (1)-(3) of Lemma 4.1. We shall show that this implies that $\Sigma$ does not contain Lannér subdiagrams of order 5. By Lemmas 4.3, 5.3, and 1.2 this would prove the lemma.

Suppose that there is a Lannér diagram $L_{0} \subset \Sigma$ of order 5 . Let $S_{0} \subset L_{0}$ be a subdiagram of type $H_{4}$ or $F_{4}$. Then $P\left(S_{0}\right)$ is a 4-polytope with at most seven facets 
and $\Sigma_{S_{0}}=\bar{S}_{0}$ (Corollary 1.1). The Coxeter diagram of any 4-polytope with seven facets contains either a Lannér subdiagram of order 3 or at least two dashed edges. The Coxeter diagram of any 4-polytope with six facets which is not a prism contains a Lannér subdiagram of order 3. Hence $P\left(S_{0}\right)$ is a either a Coxeter 4-prism or a Coxeter 4-simplex.

By Lemma 5.6, we may assume that $P\left(S_{0}\right)$ is a prism. Consider two cases.

Case 1. $P\left(S_{0}\right)$ is a prism with Coxeter diagram $\Sigma_{S_{0}}$, different from

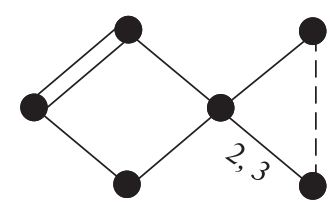

where the label " 2,3 " indicates that the corresponding nodes are either joined by a simple edge or are not joined.

Examining the list of Coxeter diagrams of 4-prisms [13, we see that $\Sigma_{S_{0}}=\bar{S}_{0}$ contains a subdiagram $S_{1}$ of type $H_{4}$ or $F_{4}$ with an adjoined dashed edge. Since there is only one dashed edge in $\Sigma$, the diagram $\Sigma_{S_{1}}=\bar{S}_{1}$ of $P\left(S_{1}\right)$ contains no dashed edges, so $P\left(S_{1}\right)$ is a simplex. Notice that $S_{0} \subset \bar{S}_{1}$. Let $u$ and $v$ be the neighbors of $S_{0}, a$ the end of the dashed edge contained in $S_{1}$, and $b$ the remaining node of $\Sigma$ not contained in $\left\langle S_{0}, S_{1}\right\rangle$. Thus, $\Sigma$ consists of the following parts:

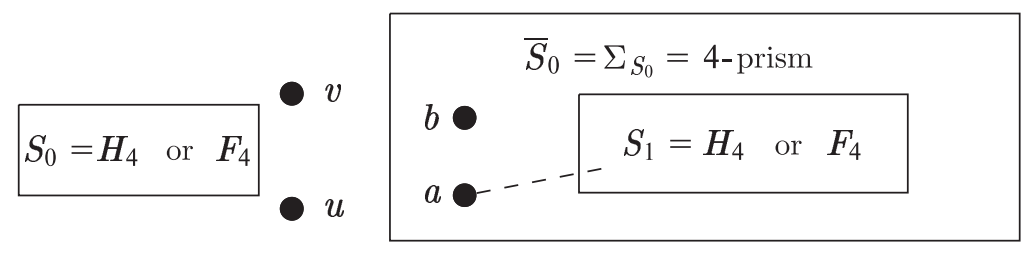

where $u$ and $v$ are neighbors of $S_{0}$, and $S_{0}$ is disjoint from $\bar{S}_{0}$. Since $P\left(S_{1}\right)$ is a simplex, $b$ and one of the nodes $u$ and $v$, say $v$, are joined with $S_{1}$, and $\left\langle S_{0}, u\right\rangle=\bar{S}_{1}$ is a Lannér diagram.

Consider the diagram $\Sigma \backslash\langle v, a\rangle$ consisting of the Lannér subdiagram $\left\langle S_{0}, u\right\rangle=\bar{S}_{1}$ and the indefinite subdiagram $\left\langle S_{1}, b\right\rangle$. These subdiagrams can be joined only by the edge $\langle u, b\rangle$, which cannot be dashed. It is not difficult to see that $\Sigma \backslash\langle v, a\rangle$ is superhyperbolic.

Case 2. $P\left(S_{0}\right)$ is a prism with Coxeter diagram $\Sigma_{S_{0}}$ of the form

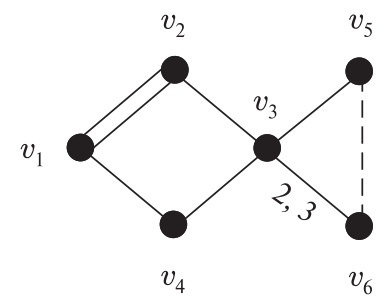


Let $S_{1} \subset \Sigma_{S_{0}}$ be a subdiagram of type $B_{4}$, for example, $S_{1}=\left\langle v_{1}, v_{2}, v_{3}, v_{5}\right\rangle$. Then $\Sigma$ consists of the following parts:

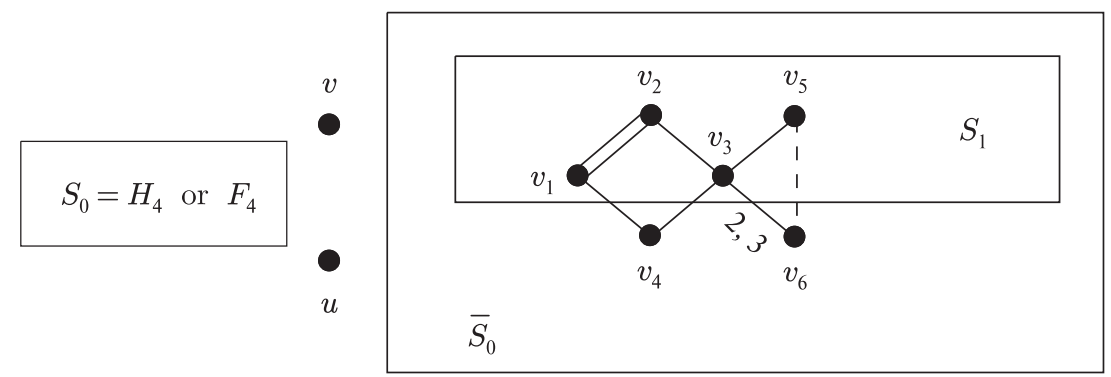

where $u$ and $v$ are neighbors of $S_{0}$, and $S_{0}$ is disjoint from $\bar{S}_{0}$. Notice that $S_{1}$ has at least two neighbors, namely, $v_{4}$ and $v_{6}$, and therefore $P\left(S_{1}\right)$ is either a prism, or an Esselmann polytope, or a simplex. Consider these three cases.

Case 2.1: $P\left(S_{1}\right)$ is an Esselmann polytope.

Then $\Sigma_{S_{1}}$ contains two disjoint Lannér subdiagrams of order 3 , whereas $\bar{S}_{1}$ contains no such diagrams. Hence $\Sigma_{S_{1}}$ contains two nodes, which are good neighbors, in $\Sigma$, of the subdiagram $S_{1}$ of type $B_{4}$. By Corollary 1.2 this is impossible (see the list of Esselmann diagrams in 9]).

Case 2.2: $P\left(S_{1}\right)$ is a prism.

As before, $u$ and $v$ are neighbors of $S_{0}$. By assumption, there is only one dashed edge in $\Sigma$, and therefore one of the nodes $u$ and $v$, say $v$, is a good neighbor of $S_{1}$, i.e., the diagram $\left\langle S_{1}, v\right\rangle$ is of type $B_{5}$. If $u$ is also a good neighbor of $S_{1}$, we obtain either a parabolic subdiagram of $\Sigma$ or a Lannér subdiagram of order 3 . Thus, $v$ is the only good neighbor of $S_{1}$.

Consider the diagram $\bar{S}_{1}=\left\langle S_{0}, u, v\right\rangle$. Since $v$ is the only node of $\bar{S}_{1}$ joined with $S_{1}$, the diagram $\bar{S}_{1}$ differs from $\Sigma_{S_{1}}$ only by the multiplicities of the edges incident to $v$. By Proposition 1.11, any simple edge of $\bar{S}_{1}$ incident to $v$ becomes a double edge in $\Sigma_{S_{1}}$, and any other edge of $\bar{S}_{1}$ incident to $v$ becomes a dashed edge. Since $P\left(S_{1}\right)$ is a prism, $\Sigma_{S_{1}}$ contains only one dashed edge. At the same time, no Coxeter diagram of a compact Coxeter 4-prism contains nodes incident to both a multiple edge and a dashed edge. Hence $v$ is joined with exactly one node of the diagram $\bar{S}_{1} \backslash v=\left\langle S_{0}, u\right\rangle$ (call it $w$ ), and $\bar{S}_{1}$ can be obtained from $\Sigma_{S_{1}}$ by replacing the dashed edge by a double or a triple edge.

Recall that $v$ is the only neighbor of $S_{1}$ contained in $\bar{S}_{1}$, and that it is joined with $v_{5}$ by a simple edge. Thus, we have either the parabolic diagram $\left\langle v_{1}, v_{2}, v_{3}, v_{5}, v, w\right\rangle$ or the subdiagram $\left\langle v_{3}, v_{5}, v, w\right\rangle$ of type $H_{4}$ with at least four neighbors.

Case 2.3: $P\left(S_{1}\right)$ is a simplex.

We use the same notation as in Case 1. The difference is that now one of the nodes $u$ and $v$, say $v$, is a bad neighbor of $S_{1}$. There is only one way to attach a bad neighbor to $S_{1}$ such that no parabolic subdiagrams or Lannér subdiagrams of order 3 would be formed: join $v$ with $v_{5}$ by a triple edge. But in that case we would have the subdiagram $\left\langle v_{2}, v_{3}, v_{5}, v\right\rangle$ of type $H_{4}$ with at least four neighbors.

This exhausts all the cases and proves the lemma.

Theorem 2. There are no compact hyperbolic Coxeter 8-polytopes with 12 facets.

Proof. Suppose $P$ is a compact hyperbolic Coxeter 8-polytope with 12 facets and $\Sigma$ is its Coxeter diagram. By Lemma 5.4, $\Sigma$ has a dashed edge $\langle v, w\rangle$. It follows from Lemma 5.7 that $P(v)$ is a 7-polytope with ten facets. By Lemma [5.8, $P(v)$ has a pair of disjoint facets. 
The combinatorial structure of $P(v)$ is encoded by a 2-dimensional Gale diagram on ten nodes with a missing face of order 2 and without large missing faces. It is not difficult to check that it must be one of the following diagrams:
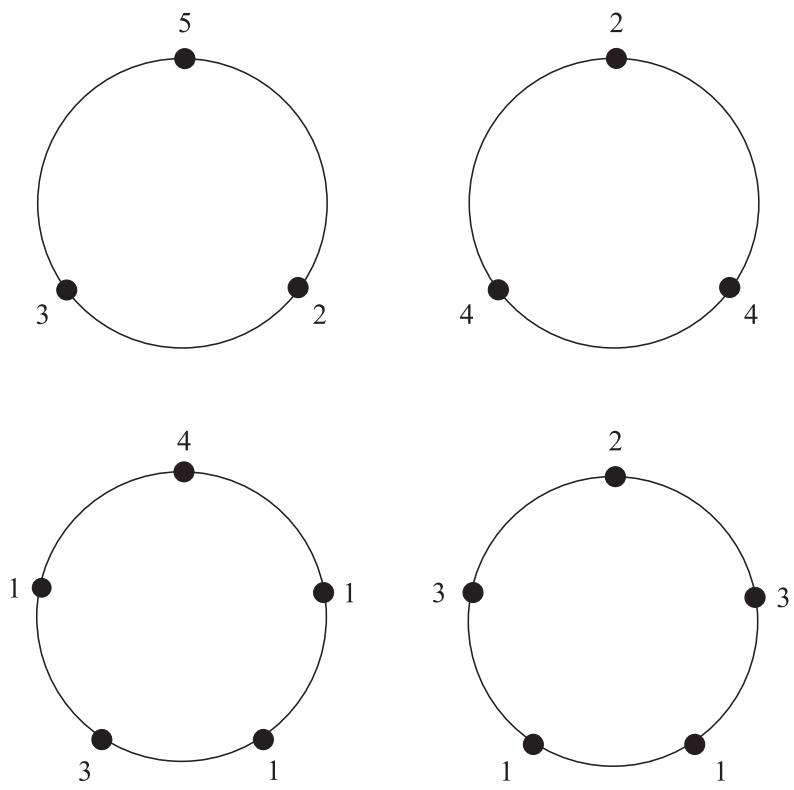

By Lemma 3.2, $\Sigma$ contains either a 0 - or a 1-lifting of one of these diagrams. By Lemma 5.3 $\Sigma$ has no Lannér subdiagrams of order 3. Since $P$ is an 8-polytope, the positive inertia index of $\Sigma$ is 8. Thus Lemmas 3.7 3.8, 3.10, and 3.11 apply, and we have that $\Sigma$ contains $\Sigma\left(P_{8}\right)$. This contradicts Lemma 5.1 and completes the proof of the theorem.

\section{Polytopes in Dimension 7}

In this section we assume that $\Sigma$ is the Coxeter diagram of a compact Coxeter 7polytope with 11 facets, and prove that $\Sigma$ coincides with $\Sigma_{P_{7}}$, where $\Sigma_{P_{7}}$ is the diagram found by Bugaenko [5] and shown in Figure 7 .

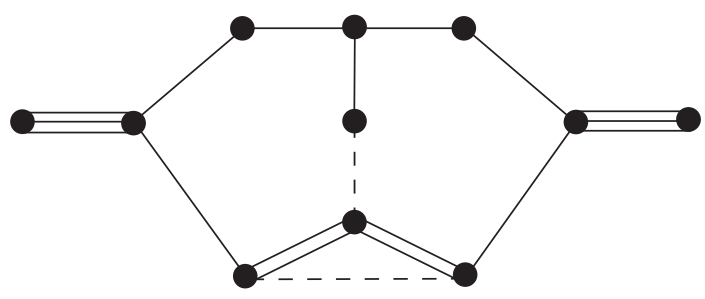

Figure 7 . The only compact Coxeter 7-polytope with 11 facets

Theorem 3. If $\Sigma$ is the Coxeter diagram of a compact Coxeter 7-polytope with 11 facets, then $\Sigma=\Sigma_{P_{7}}$.

The proof in general is similar to the proofs in larger dimensions but it is rather long and involved, with many cases to examine. We show that $\Sigma$ contains at least one subdiagram of type $F_{4}$ or $H_{4}$, and finish the proof by presenting Lemmas 6.6 6.9, which deal with subdiagrams of type $F_{4}$ and $H_{4}$ and take up most of the proof. 
We say that a Coxeter diagram satisfies the signature condition if it is admissible and its positive inertia index is at most 7 .

Recall that if $u, v \in \Sigma$, then $\langle u, v\rangle=m$ ( $\infty$ or 2 ) means that $u$ and $v$ are joined by an edge of multiplicity $m-2$ (respectively, dashed or empty edge).

6.1. Existence of subdiagrams of type $F_{4}$ or $H_{4}$. Here we establish the following properties of $\Sigma$ :

- any node of $\Sigma$ is incident to at most one dashed edge (Lemma 6.2);

- $\Sigma$ contains no subdiagrams of type $G_{2}^{(k)}$ with $k>5$ (Lemma 6.4);

- $\Sigma$ contains at least one subdiagram of type $F_{4}$ or $H_{4}$ (Lemma 6.5), and any such subdiagram has at least two bad neighbors (Lemma 6.3).

Recall that an elliptic subdiagram of $\Sigma$ has at most three bad neighbors (Lemma 1.3).

Lemma 6.1. A subdiagram of type $G_{2}^{(k)}$ with $k>3$ has at most two bad neighbors.

Proof. Suppose that $S_{0} \subset \Sigma$ is a subdiagram of type $G_{2}^{(k)}, k>3$, with three bad neighbors. Then $P\left(S_{0}\right)$ is a 5 -simplex, which is impossible.

Lemma 6.2. Any node of $\Sigma$ is incident to at most one dashed edge.

Proof. Suppose that a node $v$ is incident to two or more dashed edges. Let $f$ be the facet of $P$ corresponding to $v$. Then $f$ is a (possibly non-Coxeter) 6-polytope with at most $6+2$ facets. By Corollary 3.1, $f$ cannot be a simplex. Therefore, by Proposition 1.7 . $f$ is a product of two simplices, i.e., $\Delta^{5} \times \Delta^{1}$, or $\Delta^{4} \times \Delta^{2}$, or $\Delta^{3} \times \Delta^{3}$. The first case is impossible, since $\Sigma$ has no large missing faces. The remaining cases are impossible, since the diagrams $\lfloor 5,3\rfloor_{1}$ and $\lfloor 4,4\rfloor_{1}$ have no 0 - and 1-liftings with positive inertia index smaller than 8 (see Lemma 3.6 and 3.11).

Lemma 6.3. Any subdiagram of $\Sigma$ of type $H_{4}$ or $F_{4}$ has at least two neighbors.

Proof. Suppose $S_{0} \subset \Sigma$ is of type $H_{4}$ or $F_{4}$. Since $\Sigma$ is connected, $S_{0}$ has at least one neighbor. Suppose that $S_{0}$ has exactly one neighbor, and call it $a$. Then $P\left(S_{0}\right)$ is a Coxeter 3-polytope with $3+3$ facets. A simple Coxeter 3-polytope with six facets can be either a cube or a frustum of a tetrahedron, i.e., a polytope with two triangular, two quadrilateral, and two pentagonal faces. The latter case is impossible for $P\left(S_{0}\right)$, since a triangular facet does not intersect the two other facets, which is impossible by Lemma 6.2 (we use the fact that $\Sigma_{S_{0}}=\bar{S}_{0} \subset \Sigma$, since $S_{0}$ is of type $H_{4}$ or $F_{4}$ ). Thus, $P\left(S_{0}\right)$ is a cube. Let $b_{1}$ and $b_{2}, c_{1}$ and $c_{2}, d_{1}$ and $d_{2}$ be the ends of the dashed edges in $\bar{S}_{0}=\Sigma_{S_{0}}$. By Lemma 1.4, $a$ is joined with each of the dashed edges $b_{1} b_{2}, c_{1} c_{2}$, and $d_{1} d_{2}$. Assume that $a$ is joined with $b_{1}, c_{1}$, and $d_{1}$.

Suppose that $\left[b_{1}, c_{1}\right] \geq 4$. Then the subdiagram $\left\langle b_{1}, c_{1}\right\rangle$ has at least three bad neighbors $\left(b_{2}, c_{2}\right.$ and $\left.a\right)$, which is impossible by Lemma 6.1 . Furthermore, $\left[b_{1}, c_{1}\right] \neq \infty$, since $P\left(S_{0}\right)$ is a cube. Hence $\left[b_{1}, c_{1}\right]=2$ or 3 . Similarly, $\left[b_{1}, d_{1}\right] \leq 3$ and $\left[c_{1}, d_{1}\right] \leq 3$.

Now suppose that $\left[a, b_{1}\right] \geq 4$. Let $S_{1}=\left\langle a, b_{1}\right\rangle$. If $\left[a, b_{1}\right] \geq 6$, then $S_{1}$ has at least three bad neighbors $\left(b_{2}, c_{1}\right.$, and $\left.d_{1}\right)$, contrary to Lemma 6.1. Hence $\left[a, b_{1}\right]=4$ or 5 . By Lemma 6.1, $S_{1}$ has at most two bad neighbors, and therefore one of the nodes $c_{1}$ and $d_{1}$ is a good neighbor of $S_{1}$ (recall that both $c_{1}$ and $d_{1}$ are joined with $a$ ). Assuming now that $c_{1}$ is a good neighbor of $S_{1}$, consider the diagram $S_{2}=\left\langle S_{1}, c_{1}\right\rangle$ of type $H_{3}$ or $B_{3}$. The diagram $S_{2}$ has at least four bad neighbors, namely $b_{2}, c_{2}, d_{1}$ and one of the nodes of $S_{0}$ (since $a$ is a neighbor of $S_{0}$ ), which is impossible by Lemma 1.3. The obtained contradiction shows that $\left[a, b_{1}\right]=3\left(\left[a, b_{1}\right] \neq \infty\right.$ by Lemma 6.2). Similarly, $\left[a, c_{1}\right]=\left[a, d_{1}\right]=3$. 
Since $\Sigma$ contains no parabolic subdiagrams and $\left[b_{1}, c_{1}\right] \leq 3$, we have $\left[b_{1}, c_{1}\right]=2$. Similarly, $\left[b_{1}, d_{1}\right]=\left[c_{1}, d_{1}\right]=2$, and therefore $\left\langle a, b_{1}, c_{1}, d_{1}\right\rangle$ is of type $D_{4}$. This diagram has at least four bad neighbors: $b_{2}, c_{2}, d_{2}$ and one of the nodes $x_{i}, 1 \leq i \leq 4$. The obtained contradiction proves the lemma.

Lemma 6.4. $\Sigma$ contains no subdiagrams of type $G_{2}^{(k)}$ with $k>5$.

Proof. Suppose that $S_{0} \subset \Sigma$ is a subdiagram of type $G_{2}^{(k)}, k>5$. Without loss of generality, we may assume that the edge of the subdiagram $S_{0}$ is of maximum multiplicity in $\Sigma$. Since $\Sigma$ is connected, $S_{0}$ has at least one (obviously, bad) neighbor. By Corollary 1.3, $S_{0}$ has at most two neighbors. Consider two cases.

a)

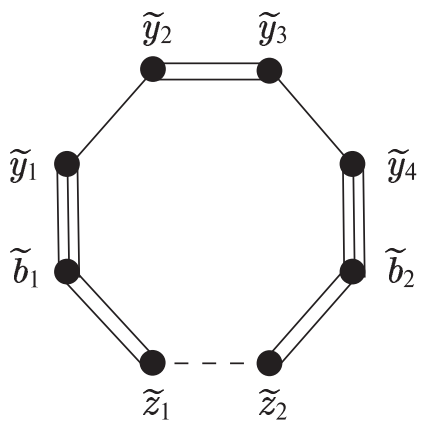

b)
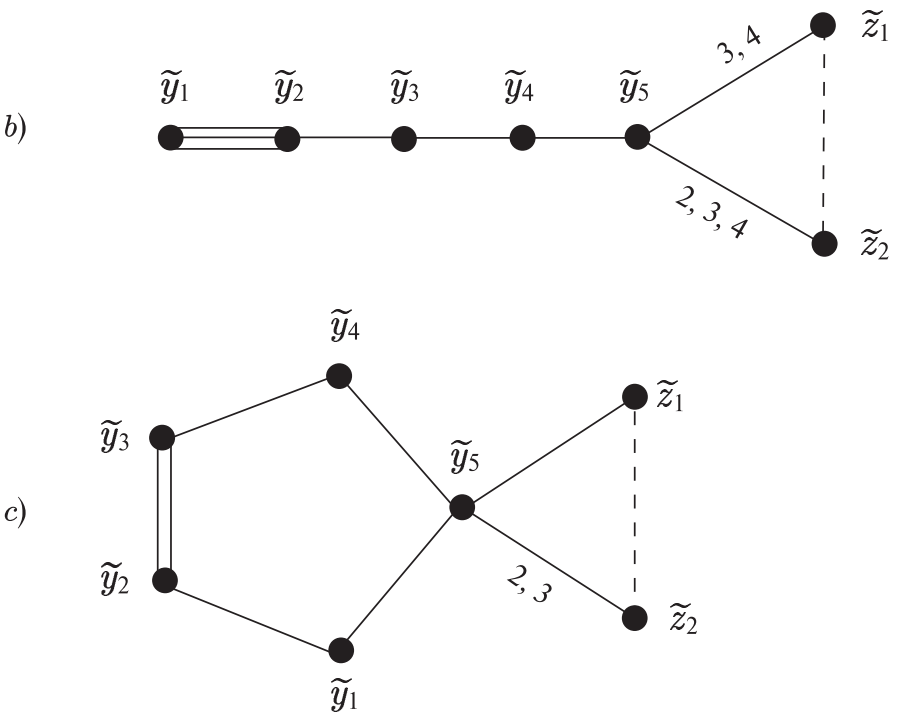

Figure 8. To the proofs of Lemmas 6.4 and 6.6

Case 1. Suppose that $S_{0}=\left\langle x_{1}, x_{2}\right\rangle$ has only one neighbor, and call it $a$. Then $P\left(S_{0}\right)$ is a 5-polytope with $5+3$ facets. By Corollary 1.1. $\Sigma_{S_{0}}=\bar{S}_{0}$. Hence, it follows from Lemma 6.2 that any node of $\Sigma_{S_{0}}$ is incident to at most one dashed edge. The list of 5-polytopes with eight facets has only one diagram satisfying this condition. We reproduce that diagram in Figure 8, a) together with the notation for its nodes. By Lemma 1.4 $a$ is joined with at least one of the nodes $z_{1}$ and $z_{2}$, say, $z_{1}$. Let $S_{1}=\left\langle x_{1}, z_{1}\right\rangle$. If $a$ is a bad neighbor of $S_{1}$, then $S_{1}$ has three bad neighbors $\left(y_{1}, z_{2}, a\right)$, which is impossible by Lemma 6.1. Thus, $a$ is a good neighbor of $S_{1}$, which implies that $\left[a, z_{1}\right]=3$ 
and that $S_{2}=\left\langle a, S_{1}\right\rangle$ is of type $B_{3}$. As $a$ is a neighbor of $S_{0}$, we may assume that $x_{1}$ is joined with $a$. If $x_{1}$ is a good neighbor of $S_{2}=\left\langle a, S_{1}\right\rangle$, then $\left\langle x_{1}, S_{2}\right\rangle$ has at least four bad neighbors (namely, $x_{2}, z_{2}, y_{1}$ and some node of the Lannér diagram $\left\langle b_{2}, y_{4}, y_{3}, y_{2}\right\rangle$ joined with $a$. The last of the above neighbors is actually bad, since $a$ is not a leaf of $\left.\left\langle x_{1}, S_{2}\right\rangle\right)$. This is impossible by Lemma 1.3, so $x_{1}$ is a bad neighbor of $S_{2}$, and $S_{2}$ has three bad neighbors $\left(x_{1}, y_{1}, z_{2}\right)$. Hence $P\left(S_{2}\right)$ is a 4 -simplex and $\Sigma_{S_{2}}$ is a Lannér diagram of order 5 . By Corollary 1.2, $\bar{S}_{2}$ is also a Lannér diagram of order 5 . At the same time, $\bar{S}_{2}=\left\langle x_{2}, b_{2}, y_{4}, y_{3}, y_{2}\right\rangle$ cannot be a Lannér diagram, since it contains the Lannér subdiagram $\left\langle b_{2}, y_{4}, y_{3}, y_{2}\right\rangle$. The obtained contradiction shows that $S_{0}$ has exactly two bad neighbors.

Case 2. Suppose that $S_{0}=\left\langle x_{1}, x_{2}\right\rangle$ has two bad neighbors, $a_{1}$ and $a_{2}$. Then $P\left(S_{0}\right)$ is a 5 -prism. Such a prism can be one of the two types shown in Figure $8, b), c)$. We denote the nodes of $\bar{S}_{0}$ as is shown in that figure. Let $S_{1}$ denote the subdiagram $\left\langle y_{1}, y_{2}, y_{3}, y_{4}\right\rangle$ of type $F_{4}$ or $H_{4}$. Since $\bar{S}_{1}=\Sigma_{S_{1}}$ contains the subdiagram $S_{0}$ of type $G_{2}^{(k)}, k>5$, the diagram $\bar{S}_{1}$ cannot be a Lannér diagram of order 4 . Therefore, the face $P\left(S_{1}\right)$ cannot be a 3 -simplex. Hence $S_{1}$ has at most two neighbors, i.e., at least one of the nodes $a_{1}$ and $a_{2}$ is not joined with $S_{1}$. We may assume that $a_{1}$ is not joined with $S_{1}$. By Lemma 1.4. we then conclude that $a_{1}$ is joined with $y_{5}$ (since $y_{5}$ belongs to the Lannér diagram $\left.\left\langle y_{1}, y_{2}, y_{3}, y_{4}, y_{5}\right\rangle \subset \bar{S}_{0}\right)$. Now we consider the cases $S_{1}=F_{4}$ and $S_{1}=H_{4}$ separately.

In the case $S_{1}=F_{4}$, consider the subdiagrams $\left\langle y_{2}, y_{3}, y_{4}, y_{5}, z_{1}\right\rangle$ and $\left\langle y_{3}, y_{2}, y_{1}, y_{5}, z_{1}\right\rangle$ of type $B_{5}$. Each of these subdiagrams has three bad neighbors $\left(a_{1}, y_{1}, z_{2}\right.$ and $a_{1}, y_{4}, z_{2}$, respectively), and therefore $a_{2}$ is not a bad neighbor of either of these diagrams. Hence $a_{2}$ cannot be joined with the Lannér diagram $\left\langle S_{1}, y_{5}\right\rangle$, contrary to Lemma 1.4.

Consider now the case $S_{1}=H_{4}$. Since $a_{1}$ is not a neighbor of $S_{1}, a_{2}$ is a neighbor of $S_{1}$; otherwise $S_{1}$ would have just one neighbor, which is impossible by Lemma 6.3. Thus, $S_{1}$ has two bad neighbors, $y_{5}$ and $a_{2}$, and $P\left(S_{1}\right)$ is a 3 -prism. This means that the diagram $\bar{S}_{1}=\Sigma_{S_{1}}$ consists of the dashed edge $\left\langle z_{1}, z_{2}\right\rangle$ and the Lannér diagram $\left\langle S_{0}, a_{1}\right\rangle$ of order 3. Hence the diagram $X=\Sigma \backslash\left\langle z_{1}, z_{2}\right\rangle=\left\langle S_{0}, a_{1}, S_{1}, y_{5}\right\rangle$ consists of the Lannér diagrams $\left\langle S_{0}, a_{1}\right\rangle$ and $\left\langle S_{1}, y_{5}\right\rangle$ joined only by the edge $a_{1} y_{5}$. If this edge is not dashed and $\left[a_{1}, y_{5}\right] \neq 5$, then $X$ is superhyperbolic. Consider the cases $\left[a_{1}, y_{5}\right]=5$ and $\left[a_{1}, y_{5}\right]=\infty$.

Case 2.1. Suppose that $\left[a_{1}, y_{5}\right]=5$. Then $\left\langle a_{1}, y_{5}, y_{4}, y_{3}\right\rangle$ is of type $H_{4}$ and has three bad neighbors, namely $z_{1}, y_{2}$ and one of the nodes $x_{1}$ and $x_{2}$, say, $x_{1}$. Hence $z_{2}$ is not a neighbor of $\left\langle a_{1}, y_{5}, y_{4}, y_{3}\right\rangle$. In particular, $\left[z_{2}, a_{1}\right]=2$, whence (Lemma 1.4) $\left[a_{1}, z_{1}\right] \neq 2$. Hence $z_{1}$ is a bad neighbor of $S_{2}=\left\langle a_{1}, y_{5}\right\rangle$, and therefore $\Sigma_{S_{2}}$ is the diagram of a 5 polytope with at most eight facets. Consider $\Sigma_{S_{2}}$. By Proposition 1.11, the subdiagram $S_{1}=\left\langle y_{1}, y_{2}, y_{3}, y_{4}\right\rangle$ of $\Sigma$ becomes, in $\Sigma_{S_{2}}$, a linear diagram of order 4 with a triple edge $\widetilde{y}_{1} \widetilde{y}_{2}$, simple edge $\widetilde{y}_{2} \widetilde{y}_{3}$ and an edge $\widetilde{y}_{3} \widetilde{y}_{4}$ labeled by 10 . But no diagram of a compact 5 -polytope with eight facets contains such a subdiagram. Thus, the case $\left[a_{1}, y_{5}\right]=5$ is impossible.

Case 2.2. Now suppose that $\left[a_{1}, y_{5}\right]=\infty$. Consider the subdiagram $S_{2}=\left\langle y_{1}, y_{2}, y_{3}\right\rangle$ of type $H_{3}$. If $a_{2}$ is a bad neighbor of $S_{2}$, then $P\left(S_{2}\right)$ is a 4-polytope with $4+3=7$ facets; moreover, $\Sigma_{S_{2}}$ contains a subdiagram $\left\langle\widetilde{x}_{1}, \widetilde{x}_{2}\right\rangle$ of type $G_{2}^{(k)}$ with $k>5$ and at least two dashed edges $\left(\widetilde{z}_{1} \widetilde{z}_{2}\right.$ and $\left.\widetilde{y}_{4} \widetilde{y}_{5}\right)$. But no 4-polytope with seven facets has these properties. Therefore, $a_{2}$ is not a bad neighbor of $S_{2}$. If $a_{2}$ is a good neighbor of $S_{2}$, then the diagram $\left\langle y_{1}, y_{2}, y_{3}, a_{2}\right\rangle$ of type $H_{4}$ has at least four bad neighbors (namely, $y_{4}$, at least one of the nodes $x_{1}$ and $x_{2}$, at least one of the nodes $z_{1}$ and $z_{2}$, and at least one of the nodes $a_{1}$ and $y_{5}$ ). Hence $a_{2}$ is not a neighbor of $S_{2}$, and, by Lemma 6.3, $a_{2}$ is joined with $y_{4}$. Consider the subdiagram $S_{3}=\left\langle y_{2}, y_{3}, y_{4}, y_{5}, z_{1}\right\rangle$ of type $A_{5}$ or $B_{5}$. 
It has three bad neighbors $\left(y_{1}, a_{1}, z_{2}\right)$, and therefore $a_{2}$ is a good neighbor of $S_{3}$. Thus $\left\langle a_{2}, S_{3}\right\rangle$ is of type $E_{6}$. But then $\left\langle a_{2}, S_{3}\right\rangle$ has at least four bad neighbors $\left(y_{1}, z_{2}, a_{1}\right.$, and one of the nodes $x_{1}$ and $x_{2}$ ). This contradicts Lemma 1.3 and completes the proof of the lemma.

Lemma 6.5. $\Sigma$ contains at least one subdiagram of type $F_{4}$ or $H_{4}$.

Proof. Suppose that $\Sigma$ contains no subdiagrams of type $F_{4}$ or $H_{4}$.

Suppose that $\Sigma$ has a subdiagram $S_{0}=\left\langle x_{1}, x_{2}\right\rangle$ of type $G_{2}^{(4)}$ or $G_{2}^{(5)}$ with a bad neighbor. Then $P\left(S_{0}\right)$ is a 5-polytope with at most eight facets. Hence $\Sigma_{S_{0}}$ contains a subdiagram of type $F_{4}$ or $H_{4}$. Corollary 1.2 implies that $\bar{S}_{0}$ also contains a subdiagram of that type, which is impossible by assumption.

It now follows (in view of Lemma 6.4) that $\Sigma$ has no Lannér diagrams of order 3. Since any Lannér diagram of order 5 contains a subdiagram of type $F_{4}$ or $H_{4}, \Sigma$ does not contain Lannér subdiagrams of order 5. By [8, Prop. 6.9], any simple polytope of dimension $d>4$ with $d+4$ facets has at least one missing face of order greater than two. Thus, $\Sigma$ contains a Lannér subdiagram $L$ of order 4 . Let $S_{0} \subset L$ be a subdiagram of type $H_{3}$ or $B_{3}$. Set $a_{1}=L \backslash S_{0}$ and $S_{0}=\left\langle x_{1}, x_{2}, x_{3}\right\rangle$.

Suppose that $S_{0}$ has three bad neighbors. Then $P\left(S_{0}\right)$ is a 4 -simplex, and $\Sigma_{S_{0}}$ (and therefore $\bar{S}_{0}$ ) contains a subdiagram of type $F_{4}$ or $H_{4}$, which is impossible by assumption.

Now suppose that $S_{0}$ has only one bad neighbor, $a_{1}$. Then $P\left(S_{0}\right)$ is a 4-polytope with $4+3$ facets. Corollary 1.2 implies that its diagram contains no subdiagrams of type $F_{4}$ and $H_{4}$. Any diagram of a 4 -polytope with $4+3$ facets that does not contain subdiagrams of type $F_{4}, H_{4}$, and $G_{2}^{(k)}(k>5)$ contains a subdiagram $\left\langle\widetilde{y}_{1}, \widetilde{y}_{2}\right\rangle$ of type $G_{2}^{(4)}$ or $G_{2}^{(5)}$ with a bad neighbor. If $S_{0}$ is of type $H_{3}$, then it has no good neighbors (since $\Sigma$ has no subdiagrams of type $H_{4}$ ), and therefore $\bar{S}_{0}=\Sigma_{S_{0}}$ contains a subdiagram $S_{1}=\left\langle y_{1}, y_{2}\right\rangle$ of type $G_{2}^{(4)}$ or $G_{2}^{(5)}$ with a bad neighbor. As we have seen before, this is impossible. Therefore, $S_{0}$ is of type $B_{3}$. Using Corollary 1.1 we have that either $S_{1} \subset \bar{S}_{0}$ is a multiple edge with a bad neighbor (which is impossible) or $\Sigma_{S_{0}}$ is one of the following diagrams:
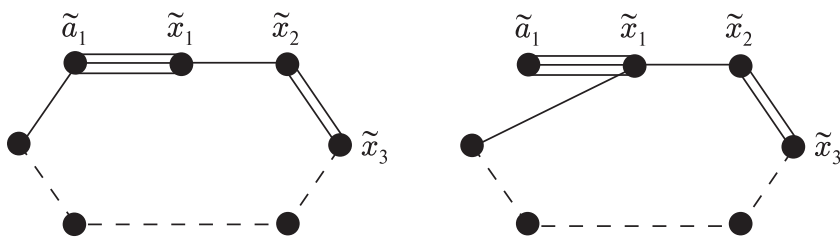

In this case the double edge $\left\langle\widetilde{y}_{1}, \widetilde{y}_{2}\right\rangle$ may become a simple edge of $\bar{S}_{0}$, which would yield a subdiagram $\left\langle y_{1}, y_{2}, y_{3}, y_{4}\right\rangle$ of type $H_{4}$ in $\bar{S}_{0} \subset \Sigma$, which is also impossible. Hence the multiple edge $\left\langle\widetilde{y}_{1}, \widetilde{y}_{2}\right\rangle$ becomes the multiple edge $\left\langle y_{1}, y_{2}\right\rangle \subset \bar{S}_{0}$, and Corollary 1.1 implies that the bad neighbor of that edge remains bad in $\bar{S}_{0}$, which is also impossible. Hence $S_{0}$ cannot have three bad neighbors.

Thus, $S_{0}$ has exactly two bad neighbors, $a_{1}$ and $a_{2}$, and $P\left(S_{0}\right)$ is an Esselmann polytope or a 4 -prism. Since $\Sigma$ contains no subdiagrams of type $F_{4}$ and $H_{4}$, we see, using Corollary [1.2, that $\Sigma_{S_{0}}$ coincides with the following diagram:

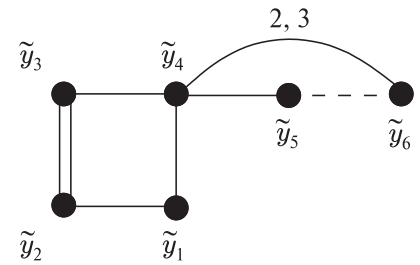


By Corollary 1.2 , the nodes of $\Sigma_{S_{0}}$ (with a possible exception of $\widetilde{y}_{6}$ in the case $\left[\widetilde{y}_{4}, \widetilde{y}_{6}\right]=2$ ) cannot be good neighbors of $S_{0}$. In particular, $\bar{S}_{0}$ contains a cyclic Lannér diagram of order 4 with exactly one double edge and without subdiagrams of type $H_{3}$. Furthermore, it is not difficult to check that the dashed edge $\left\langle\widetilde{y}_{5}, \widetilde{y}_{6}\right\rangle$ of $\Sigma_{S_{0}}$ becomes the dashed edge $\left\langle y_{5}, y_{6}\right\rangle$ in $\bar{S}_{0}$ (since otherwise $\left[y_{4}, y_{6}\right]=2$ and if $y_{5} y_{6}$ is a triple edge, then $\left[y_{4}, y_{6}\right]=2$ and $\left\langle y_{3}, y_{4}, y_{5}, y_{6}\right\rangle \subset \bar{S}_{0}$ is a subdiagram of type $H_{4}$, which is impossible by assumption; if $y_{5} y_{6}$ is a double edge, then $\left\langle y_{2}, y_{3}, y_{4}, y_{5}, y_{6}\right\rangle \subset \Sigma$ is a parabolic subdiagram of type $\widetilde{C}_{4}$, which is also impossible; if $y_{5} y_{6}$ is a simple edge, then $\left\langle y_{5}, y_{6}, S_{0}\right\rangle$ is of type $B_{5}$ and, by Proposition 1.11, $\widetilde{y}_{5} \widetilde{y}_{6}$ must be a double edge, not dashed, in $\left.\Sigma_{S_{0}}\right)$. Thus, $\bar{S}_{0}$ consists of a dashed edge and a cyclic Lannér subdiagram of order 4 , and $\bar{S}_{0}=\Sigma_{S_{0}}$.

Consider the diagram $S_{1}=\left\langle y_{1}, y_{2}, y_{3}\right\rangle$, which is a subdiagram of type $B_{3}$ in the Lannér diagram $\left\langle y_{1}, y_{2}, y_{3}, y_{4}\right\rangle$ of order 4 . Arguing as for $\bar{S}_{0}$, we have that $S_{1}$ has exactly two bad neighbors $\left(y_{4}\right.$ and one of the nodes $a_{1}$ and $\left.a_{2}\right)$ and that $\bar{S}_{1}$ consists of a dashed edge $y_{5} y_{6}$ and a cyclic Lannér diagram $\left(\left\langle S_{0}, a_{2}\right\rangle\right.$ or $\left\langle S_{0}, a_{1}\right\rangle$, respectively). Without loss of generality, we may assume that $a_{2}$ is a bad neighbor of $S_{1}$ (and $\left\langle a_{1}, S_{0}\right\rangle$ is a cyclic Lannér diagram). By Corollary [1.2, $a_{1}$ cannot be a good neighbor of $S_{1}$ and, by Lemma 1.4, $a_{1}$ is joined with $y_{4}$. If $\left[a_{1}, y_{4}\right]=3$ or 4 , then $\left\langle x_{1}, x_{2}, a_{1}, y_{2}, y_{3}, y_{4}\right\rangle$ contains a parabolic subdiagram of type $\widetilde{B}_{5}$ or $\widetilde{B}_{3}$. If $\left[a_{1}, y_{4}\right]=5$, then $\left\langle x_{2}, x_{3}, a_{1}, y_{4}\right\rangle$ is of type $H_{4}$. Therefore, $\left[a_{1}, y_{4}\right]=\infty$.

Consider the diagram $S_{2}=\left\langle y_{2}, y_{3}, y_{4}\right\rangle$. Since it has two bad neighbors $\left(a_{1}\right.$ and $\left.y_{1}\right)$, the node $a_{2}$ cannot be a bad neighbor of $S_{2}$ (we are applying the results proved for the diagram $S_{0}$ to the diagram $S_{2}$ ). Hence $a_{2}$ is joined with $y_{1}$, and, repeating the foregoing arguments, we have $\left[a_{2}, y_{1}\right]=\infty$. But then the diagram $\left\langle y_{1}, y_{3}, y_{4}, y_{5}\right\rangle$ of type $D_{4}$ has four bad neighbors, namely $a_{1}, a_{2}, y_{2}, y_{6}$, which is impossible.

6.2. Subdiagrams of type $F_{4}$ and $H_{4}$. Thus, $\Sigma$ contains at least one subdiagram of type $F_{4}$ or $H_{4}$. In Lemmas 6.6 and 6.7 we shall prove that such subdiagrams always have three neighbors. Next, in Lemma 6.8 we shall show that $\Sigma$ contains no subdiagrams of type $F_{4}$. Thus $\Sigma$ contains a subdiagram of type $H_{4}$, and we shall finish the proof of Theorem 3 by Lemma 6.9, which shows that $\Sigma=\Sigma_{P_{7}}$. We remark that the proofs of Lemmas 6.7 and 6.9, dealing with subdiagrams of type $H_{4}$, are much longer than the proofs of the similar Lemmas 6.6 and 6.8 dealing with subdiagrams of type $F_{4}$. A possible reason for that is the fact that $H_{4}$ is contained in many more diagrams of $d$-polytopes with at most $d+3$ facets than $F_{4}$.

Lemma 6.6. Any subdiagram of type $F_{4}$ has exactly three neighbors.

Proof. Assume the contrary. By Lemma 6.3 this means that $\Sigma$ contains a subdiagram $S_{0}$ of type $F_{4}$ with two neighbors. Then $P\left(S_{0}\right)$ is a 3-prism, and $\Sigma_{S_{0}}=\bar{S}_{0}$ consists of a dashed edge (denote it $z_{1} z_{2}$ ) and a Lannér subdiagrams of order 3 (denote it $L=$ $\left.\left\langle x_{1}, x_{2}, x_{3}\right\rangle\right)$ containing a multiple edge. Assuming that an edge $x_{1} x_{2}$ is of maximal multiplicity in $L$, let $S_{1}=\left\langle x_{1}, x_{2}\right\rangle$. This diagram has at least one bad neighbor, $x_{3}$. By Lemma 6.1, $S_{1}$ has either one or two bad neighbors.

Suppose that $S_{1}$ has only one bad neighbor $x_{3}$. Then $P\left(S_{1}\right)$ is a 5 -polytope with $5+3=$ 8 facets. The diagram $S_{0}$ is of type $F_{4}$ and is not joined with $S_{1}$. Therefore, $\Sigma_{S_{1}}$ contains a subdiagram of type $F_{4}$, and $\Sigma_{S_{1}}$ is the diagram shown in Figure 8, a). Let $y_{1}, y_{2}, y_{3}, y_{4}$ be the nodes of $S_{0} \subset \bar{S}_{1}$, and $b_{1}, b_{2}$ the neighbors of $S_{0}$. By Corollary 1.2, the nodes $b_{1}$ and $b_{2}$ cannot be good neighbors of $S_{1}$. Since the Lannér diagram $\left\langle S_{1}, x_{3}\right\rangle$ must be joined with the Lannér diagram $\left\langle b_{1}, y_{1}, y_{2}, y_{3}\right\rangle$, the node $x_{3}$ is joined with $b_{1}$. Similarly, $x_{3}$ is joined with $b_{2}$. Consider the diagram $S_{2}=\left\langle b_{1}, y_{1}, y_{2}\right\rangle$ of type $H_{3}$. It has three bad neighbors $\left(y_{3}, x_{3}\right.$, and $\left.z_{1}\right)$ and no good ones. Therefore, $P\left(S_{2}\right)$ is a 4 -simplex and $\bar{S}_{2}=\Sigma_{S_{2}}=\left\langle y_{4}, b_{2}, z_{2}, x_{1}, x_{2}\right\rangle$ is a Lannér diagram of order 5 . Since $y_{4} b_{2}$ and $x_{1} x_{2}$ are 
disjoint multiple edges, $\bar{S}_{2}$ is a linear Lannér diagram (with edges labeled by $5,3,3$, 4 or $5,3,3,5)$. Since $\left[y_{4}, b_{2}\right]=5$, the subdiagram $\left\langle y_{4}, b_{2}, z_{2}, x_{1}\right\rangle$ (or $\left.\left\langle y_{4}, b_{2}, z_{2}, x_{2}\right\rangle\right)$ is of type $H_{4}$. Thus, the simple edge $b_{2} z_{2}$ of $\Sigma$ becomes a double edge $\widetilde{b}_{2} \widetilde{z}_{2}$ in $\Sigma_{S_{1}}$. By Corollary [1.1, this means that $\left[x_{1}, x_{2}\right]=4$. Since $x_{1} x_{2}$ is of maximum multiplicity in the Lannér diagram $\left\langle x_{1}, x_{2}, x_{3}\right\rangle$, we see that $x_{3}$ is joined with both $x_{1}$ and $x_{2}$. Hence the diagram $\left\langle y_{4}, b_{2}, z_{2}, x_{1}\right\rangle$ (or $\left\langle y_{4}, b_{2}, z_{2}, x_{2}\right\rangle$ ) is of type $H_{4}$ with at least four neighbors: $y_{3}, x_{2}$ (or $\left.x_{1}\right), x_{3}$, and $z_{1}$, which is impossible.

The obtained contradiction shows that $S_{1}$ has two bad neighbors, namely $x_{3}$ and some other node $a_{1}$, i.e., $P\left(S_{1}\right)$ is a 5 -prism, containing a subdiagram of type $F_{4}$ (see Figure 8. c) for the notation). Recall that $a_{1} \notin \bar{S}_{1}$, and therefore $a_{1}$ is a bad neighbor of $S_{0}=$ $\left\langle y_{1}, y_{2}, y_{3}, y_{4}\right\rangle$. Consider the diagrams $\left\langle y_{2}, y_{3}, y_{4}, y_{5}, z_{1}\right\rangle$ and $\left\langle y_{3}, y_{2}, y_{1}, y_{5}, z_{1}\right\rangle$ of type $B_{5}$. The node $a_{1}$ is a bad neighbor of at least one of these diagrams, say $\left\langle y_{2}, y_{3}, y_{4}, y_{5}, z_{1}\right\rangle$. Furthermore, by Lemma 1.4, the node $y_{5}$ is joined with the Lannér subdiagram $\left\langle x_{1}, x_{2}, x_{3}\right\rangle \subset$ $\bar{S}_{0}$. This means that $\left\langle y_{2}, y_{3}, y_{4}, y_{5}, z_{1}\right\rangle$ has at least four bad neighbors $\left(y_{1}, a_{1}, z_{2}\right.$, and one of the nodes $\left.x_{1}, x_{2}, x_{3}\right)$. The obtained contradiction completes the proof of the lemma.

Lemma 6.7. Any subdiagram of type $H_{4}$ has exactly three neighbors.

Proof. Assume the contrary. By Lemma 6.3, this means that $\Sigma$ has a subdiagram $S_{0}$ of type $H_{4}$ with two neighbors. Then $P\left(S_{0}\right)$ is a 3-prism, and $\Sigma_{S_{0}}=\bar{S}_{0}$ consists of a dashed edge (call it $z_{1} z_{2}$ ) and a Lannér subdiagram of order 3 (denote it $L=\left\langle y_{1}, y_{2}, y_{3}\right\rangle$ ) containing a multiple edge. Assuming that the edge $y_{1} y_{2}$ is of maximum multiplicity in $L$, let $S_{1}=\left\langle y_{1}, y_{2}\right\rangle$. By Lemma 6.1 $S_{1}$ can have one or two neighbors.

Case 1. Suppose that $S_{1}$ has only one bad neighbor, $y_{3}$. Then $P\left(S_{1}\right)$ is a 5-polytope with $5+3=8$ facets. Since $\Sigma_{S_{1}}$ contains a subdiagram $S_{0}$ of type $H_{4}$, the diagram $\Sigma_{S_{1}}$ has one of the three types $a$ ), $b$ ), and $c$ ) shown in Figure 9 . Let $x_{1}, x_{2}, x_{3}, x_{4}$ be the nodes of $S_{0} \subset \bar{S}_{1}$, and $a_{1}, a_{2}$ the neighbors of $S_{0}$ (see Figure 9). We now examine the types $a), b)$, and $c$ ) of $\Sigma_{S_{1}}$ separately.

Case 1.1. Suppose that $\Sigma_{S_{1}}$ is the diagram shown in Figure 9, a). Recall that $z_{1} z_{2}$ is a dashed edge of $\Sigma$, and therefore Lemma 6.2 implies that $a_{1}$ is a good neighbor of $S_{1}$. Without loss of generality, we may assume that $\left[a_{1}, y_{1}\right]=3$ and $\left[a_{1}, y_{2}\right]=2$. On the other hand, $a_{2}$ is not a good neighbor of $S_{1}=\left\langle y_{1}, y_{2}\right\rangle$, since $\left[a_{2}, z_{2}\right]=5$ (see Corollary [1.2). Therefore, $a_{2}$ is joined with $y_{3}$ (otherwise the Lannér diagrams $\left\langle x_{1}, x_{2}, x_{3}, x_{4}, a_{2}\right\rangle$ and $L=\left\langle S_{1}, y_{3}\right\rangle$ would not be joined). Notice that the diagram $S_{2}=\left\langle z_{2}, a_{2}, x_{4}, x_{3}\right\rangle$ of type $H_{4}$ has three neighbors $\left(x_{2}, z_{1}, y_{3}\right)$, and therefore $P\left(S_{2}\right)$ is a 3 -simplex and $\bar{S}_{2}=$ $\left\langle x_{1}, a_{1}, y_{1}, y_{2}\right\rangle$ is a Lannér diagram. Furthermore, $\bar{S}_{2}$ is a linear Lannér diagram, since $x_{1}$ is not joined with $\left\langle y_{1}, y_{2}\right\rangle$, and $a_{1}$ is not joined with $y_{2}$ and is joined with $y_{1}$ by a simple edge. Hence $\left[x_{1}, a_{1}\right]=4$ or 5 . Consider the diagram $S_{3}=\left\langle x_{1}, a_{1}, y_{1}\right\rangle$ of type $H_{3}$ or $B_{3}$. It has three bad neighbors $\left(x_{2}, z_{1}, y_{2}\right)$, and therefore $P\left(S_{3}\right)$ is a 4 -simplex and $\Sigma_{S_{3}}$ is a Lannér diagram of order 5 . Thus, by Corollary [1.2, $\bar{S}_{0}$ is also a Lannér diagram. However, in $\bar{S}_{3}=\left\langle x_{3}, x_{4}, a_{2}, z_{2}, y_{3}\right\rangle$ the valency of the end $a_{2}$ of the triple edge $z_{2} a_{2}$ cannot be less than 3 , which is impossible in a Lannér diagram of order 5 .

Case 1.2. Suppose that $\Sigma_{S_{1}}$ is one of the diagrams shown in Figure 9, b) or c). Then neither $a_{1}$ nor $a_{2}$ can be a good neighbor of $S_{1}$ (Corollary 1.2). Hence, by Lemma 6.2, both $z_{1}$ and $z_{2}$ are good neighbors of $S_{1}$ (recall that $z_{1} z_{2}$ is a dashed edge of $\Sigma$ ). By Lemma 6.4, $\left[a_{i}, z_{i}\right]=3,4$, or $5(i=1,2)$. If $\left[a_{1}, z_{1}\right]=5$, then the diagram $\left\langle z_{1}, a_{1}, x_{4}, x_{3}\right\rangle$ is of type $H_{4}$ and has at least four neighbors $\left(x_{2}, z_{2}, a_{2}\right.$, and at least one of the nodes $y_{1}$ and $\left.y_{2}\right)$. If $\left[a_{1}, z_{1}\right]=4$, then the diagram $\left\langle z_{1}, a_{1}, x_{4}, x_{3}, x_{2}\right\rangle$ is of type $B_{5}$ and has at least four bad neighbors $\left(x_{1}, z_{2}, a_{2}\right.$, and at least one of the nodes $y_{1}$ and $\left.y_{2}\right)$. Finally, if 


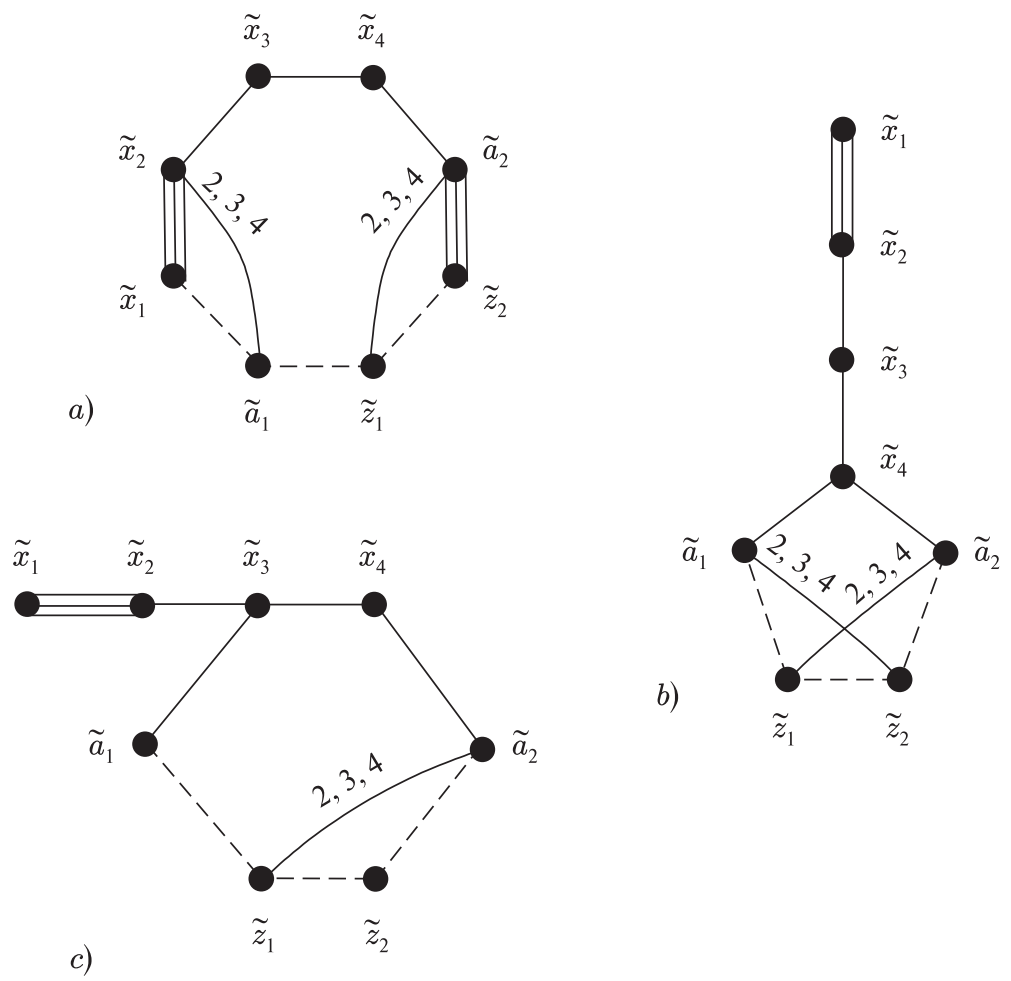

Figure 9. Possibilities for $\Sigma_{S_{1}}$ (Case 1)

$\left[a_{1}, z_{1}\right]=3$, then the edge $\widetilde{a}_{1} \widetilde{z}_{1}$ of $\Sigma_{S_{1}}$ should be either a double edge or an edge labeled by 10 , but not a dashed edge.

Case 2. Suppose that $S_{1}$ has exactly two bad neighbors: $y_{3}$ and one of the nodes $a_{1}$ and $a_{2}$, say $a_{1}$. Therefore, $P\left(S_{1}\right)$ is a 5 -prism, whose diagram contains a subdiagram of type $H_{4}$, i.e., $\Sigma_{S_{1}}$ is of the form

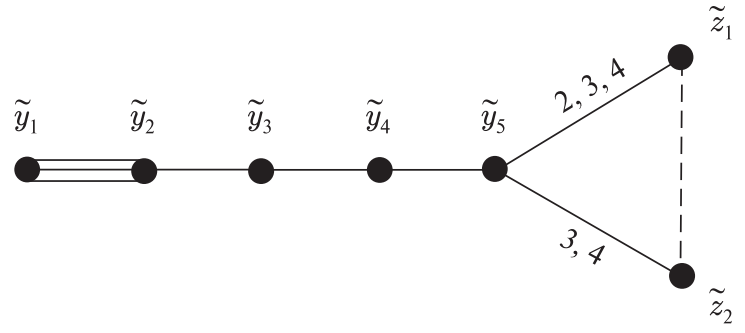

By Corollary 1.2, $a_{2}$ cannot be a good neighbor of $S_{1}$, and therefore (Lemma 1.4) $a_{2}$ is joined with $y_{3}$. If $\left[a_{2}, y_{3}\right] \neq \infty$, then the diagram $\left\langle S_{0}, a_{2}, y_{3}, S_{1}\right\rangle$ is superhyperbolic (since $\Sigma$ contains no subdiagrams of type $G_{2}^{(k)}$ with $k>5$ and $y_{1} y_{2}$ has maximum multiplicity in $\left.L=\left\langle y_{1}, y_{2}, y_{3}\right\rangle\right)$. Hence $\left[a_{2}, y_{3}\right]=\infty$. Set $S_{4}=\left\langle x_{1}, x_{2}, x_{3}\right\rangle$ and consider three cases: either the node $a_{1}$ is a bad neighbor of $S_{4}$, or $a_{1}$ is a good neighbor of $S_{4}$, or $a_{1}$ is not joined with $S_{4}$.

Case 2.1. If $a_{1}$ is a bad neighbor of $S_{4}$, then $P\left(S_{4}\right)$ is a 4-polytope with $4+3$ facets, and $\Sigma_{S_{4}}$ contains at least three dashed edges, namely $\widetilde{z}_{1} \widetilde{z}_{2}, \widetilde{a}_{2} \widetilde{y}_{3}$, and $\widetilde{x}_{4} \widetilde{a}_{2}$. But no diagram of a 4-polytope with $4+3$ facets has more than three dashed edges; moreover, in any 
diagram with exactly three dashed edges, each dashed edge has a node incident to some other dashed edge. However, the edge $\widetilde{z}_{1} \widetilde{z}_{2}$ has no nodes incident to $\widetilde{a}_{2} \widetilde{y}_{3}$ and $\widetilde{x}_{4} \widetilde{a}_{2}$, a contradiction.

Case 2.2. Let $a_{1}$ be a good neighbor of $S_{4}$. Consider the diagram $S_{5}=\left\langle x_{1}, x_{2}, x_{3}, a_{2}\right\rangle$ of type $H_{4}$. It has three bad neighbors: $x_{4}$, one of $y_{1}$ and $y_{2}$ (say, $y_{1}$ ), and one of $z_{1}$ and $z_{2}$. Hence $y_{2}$ cannot be a neighbor of $S_{5}$ and, in particular, $\left[a_{1}, y_{2}\right]=2$ and $\left[a_{1}, x_{1}\right]=2$. On the other hand, $a_{1}$ is a bad neighbor of $y_{1} y_{2}$, and therefore $\left[a_{1}, y_{1}\right] \in\{4,5, \infty\}$.

Consider the diagram $S_{6}=\left\langle x_{2}, x_{3}, x_{4}, a_{2}, z_{1}\right\rangle$ of type $A_{5}$ or $B_{5}$. Since $a_{1}$ is a neighbor of $S_{0}=\left\langle x_{1}, x_{2}, x_{3}, x_{4}\right\rangle$ and, as we have shown before, $\left[a_{1}, x_{1}\right]=2$, we see that $a_{1}$ is a neighbor of $S_{6}$. Since $S_{6}$ already has three bad neighbors $\left(x_{1}, z_{2}, y_{3}\right)$, the node $a_{1}$ is a good neighbor of $S_{6}$. Hence $\left\langle a_{1}, S_{6}\right\rangle$ is a diagram of type $D_{6}$ with three bad neighbors $x_{1}, z_{2}, y_{3}$. However, $\left[a_{1}, y_{1}\right]=4,5$, or $\infty$, and therefore $y_{1}$ is also a bad neighbor of $\left\langle a_{1}, S_{6}\right\rangle$, which is impossible.

Case 2.3. If $a_{1}$ is not a neighbor of $S_{4}=\left\langle x_{1}, x_{2}, x_{3}\right\rangle$, then $a_{1}$ is joined with $x_{4}$ (as a neighbor of $\left.S_{0}=\left\langle x_{1}, x_{2}, x_{3}, x_{4}\right\rangle\right)$. Consider the diagram $S_{7}=\left\langle x_{2}, x_{3}, x_{4}, a_{2}, z_{1}\right\rangle$ of type $A_{5}$ or $B_{5}$. It has three bad neighbors, $x_{1}, z_{2}, y_{3}$. Hence $a_{1}$ is a good neighbor of $S_{7}$, and $\left\langle S_{7}, a_{1}\right\rangle$ is of type $E_{6}$. However, $\left\langle S_{7}, a_{1}\right\rangle$ has four bad neighbors: $x_{1}, z_{2}, y_{3}$, and one of $y_{1}$ and $y_{2}$ (since, by assumption, $a_{1}$ is a bad neighbor of $S_{1}$ ).

Thus $S_{1}$ cannot have two bad neighbors, and the lemma is proved.

Lemma 6.8. $\Sigma$ has no subdiagrams of type $F_{4}$.

Proof. Let $S_{0}=\left\langle x_{1}, x_{2}, x_{3}, x_{4}\right\rangle \subset \Sigma$ be a subdiagram of type $F_{4}$. By Lemma 6.6 $S_{0}$ has exactly three neighbors; call them $a_{1}, a_{2}$, and $a_{3}$. Then $P\left(S_{0}\right)$ is a 3-simplex, and $\bar{S}_{0}=\Sigma_{S_{0}}=\left\langle y_{1}, y_{2}, y_{3}, y_{4}\right\rangle$ is a Lannér diagram of order 4 . Let $S_{1}=\left\langle y_{1}, y_{2}, y_{3}\right\rangle \subset \bar{S}_{0}$ be a subdiagram of type $H_{3}$ or $B_{3}$, and $\left\langle S_{2}, \bar{S}_{0}\right\rangle$ another such subdiagram (it exists by Lemma 1.5); we may assume that $S_{2}=\left\langle y_{2}, y_{3}, y_{4}\right\rangle$.

The diagram $S_{1}$ has at least one bad neighbor, $y_{4}$. Consider the three cases when $S_{1}$ has one, two, or three bad neighbors, respectively.

Case 1. Suppose that $S_{1}$ has only one bad neighbor $y_{4}$. Then $P\left(S_{1}\right)$ is a 4-polytope with $4+3$ facets.

Assume in addition that each of the diagrams $\left\langle a_{i}, S_{0}, \bar{S}_{0}\right\rangle, i \in\{1,2,3\}$, contains a dashed edge. Then each $a_{i}(i=1,2,3)$ is incident to a dashed edge. Consider the other ends of those dashed edges. If $S_{0}$ contains three ends of dashed edges, then $\Sigma_{S_{1}}$ contains three dashed edges with pairwise distinct ends (since $S_{0} \subset \Sigma_{S_{1}}$ and $a_{i} \in \Sigma_{S_{1}}$ for all $i=1,2,3)$. However, there are no such diagrams of 4-polytopes with seven facets. Therefore, at least one of the ends of the dashed edges belongs to $\bar{S}_{0}$. Since $a_{i} \in \Sigma_{S_{1}}$ $(i=1,2,3)$, the diagram $S_{1}$ contains no end of a dashed edge. Hence $y_{4}$ is the only end of a dashed edge in $\bar{S}_{0}$. Then $S_{2}=\left\langle y_{2}, y_{3}, y_{4}\right\rangle$ has at least two bad neighbors (namely, $y_{1}$ and some node $a_{j}$ such that $\left.\left[a_{j}, y_{4}\right]=\infty\right)$. If $S_{2}$ has exactly two bad neighbors, then $\Sigma_{S_{2}}$ is either the diagram of a 4-prism or the diagram of an Esselmann polytope. However, $\Sigma_{S_{2}}$ contains two dashed edges, which is impossible. If $S_{2}$ has three bad neighbors, then $\Sigma_{S_{2}}$ is the diagram of a 4-simplex, but $\Sigma_{S_{2}}$ contains at least one dashed edge.

Thus, we may assume that the diagram $\left\langle a_{1}, S_{0}, \bar{S}_{0}\right\rangle$ contains no dashed edges. By Lemma 6.4, we have only finitely many possibilities for the diagram $\left\langle a_{1}, S_{0}, \bar{S}_{0}\right\rangle$. The only two diagrams satisfying the signature condition and the assumption that $S_{1}$ has only one bad neighbor are shown in Figure 10.

For the diagram shown in Figure [10, $a$ ), consider the subdiagram $S_{3}=\left\langle a_{1}, y_{4}\right\rangle$ with two bad neighbors. Then $\Sigma_{S_{3}}$ is the diagram of a 5-prism. However, $\Sigma_{S_{3}}$ contains the subdiagram $\left\langle\widetilde{x}_{1}, \widetilde{x}_{2}, \widetilde{x}_{3}, \widetilde{y}_{1}, \widetilde{y}_{2}\right\rangle$ of type $B_{3}+B_{2}$, which is not a subdiagram of the diagram of any 5-prism. 
Consider now the diagram shown in Figure 10, b). The subdiagram $S_{4}=\left\langle x_{4}, a_{1}, y_{3}, y_{2}\right\rangle$ is of type $H_{4}$ and has three bad neighbors, $x_{3}, y_{4}$, and $y_{1}$. Hence $a_{2}$ and $a_{3}$ are not neighbors of $S_{4}$.

Suppose that $\left[a_{2}, y_{4}\right] \neq 0$. Then $S_{5}=\left\langle a_{1}, y_{3}, y_{4}\right\rangle$ is of type $H_{3}$ and has three bad neighbors $\left(x_{4}, y_{2}\right.$, and $a_{2}$ ). Therefore $\left[a_{3}, y_{4}\right]=2$ and $\left[a_{3}, y_{1}\right] \neq 0$ (apply Lemma 1.4 to the Lannér diagram $\left.L=\left\langle y_{1}, y_{2}, y_{3}, y_{4}\right\rangle\right)$. Furthermore, $P\left(S_{5}\right)$ is a simplex and $\Sigma_{S_{5}}$ is a Lannér diagram of order 5. By Corollary 1.2, the diagram $\bar{S}_{5}$ is also Lannér. Since $\bar{S}_{5}$ contains a subdiagram $S_{0}$ of type $F_{4}$, we have that $\bar{S}_{0}$ is a cyclic Lannér subdiagram and $\left[a_{3}, x_{1}\right]=\left[a_{3}, x_{4}\right]=3$. However, in this case the diagram $\left\langle x_{1}, a_{3}, x_{4}, a_{1}\right\rangle$ is of type $H_{4}$ and has at least four neighbors $\left(x_{2}, x_{3}, y_{3}\right.$, and $y_{1}$, which is joined with $\left.a_{3}\right)$. The obtained contradiction shows that $\left[a_{2}, y_{4}\right]=0$. Similarly, $\left[a_{3}, y_{4}\right]=0$.

By Lemma 1.4 the nodes $a_{2}$ and $a_{3}$ are joined with the Lannér diagram $\left\langle y_{1}, y_{2}, y_{3}, y_{4}\right\rangle$, and therefore $a_{2}$ and $a_{3}$ are joined with $y_{1}$. However, $\left\langle a_{1}, y_{3}, y_{2}, y_{1}\right\rangle$ is a diagram of type $B_{4}$ and has four bad neighbors, $\left(a_{2}, a_{3}, x_{4}\right.$ and $\left.y_{4}\right)$, which is impossible.

a)

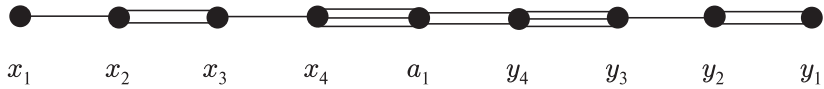

b)

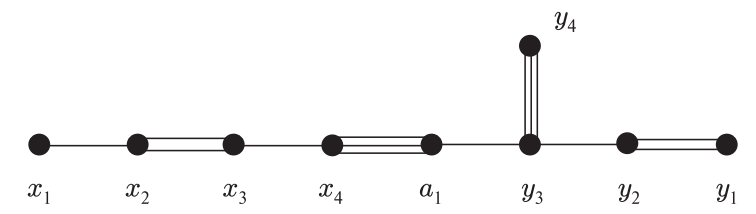

Figure 10. Case 1: two possibilities for $\left\langle a_{1}, S_{0}, \bar{S}_{0}\right\rangle$

Case 2. Suppose that $S_{1}$ has two bad neighbors, $y_{4}$ and $a_{1}$. Then $P\left(S_{1}\right)$ is a either an Esselmann polytope or a 4 -prism. Notice that $\Sigma_{S_{1}}$ contains a subdiagram $S_{0}$ of type $F_{4}$ and contains no subdiagrams of type $G_{2}^{(k)}$ with $k>5$ (see Corollary 1.1). There are only two prisms and one Esselmann polytope (see Figure 11) satisfying these conditions. We consider these polytopes separately.

Case 2.1. Let $\Sigma_{S_{1}}$ be the diagram shown in Figure 11, a). Then, by Corollary 1.2, $\Sigma_{S_{1}}=\bar{S}_{1}$. The node $a_{1}$ is a neighbor of $S_{0}=\left\langle x_{1}, x_{2}, x_{3}, x_{4}\right\rangle$. Without loss of generality, we may assume that $a_{1}$ is a neighbor of $\left\langle x_{1}, x_{2}, x_{3}\right\rangle$. Then the subdiagram $\left\langle x_{1}, x_{2}, x_{3}, a_{3}\right\rangle$ is of type $F_{4}$ and has four bad neighbors $\left(x_{4}, a_{1}, a_{2}\right.$, and some node of $\bar{S}_{0}$ joined with $a_{3}$ ), which is impossible.

Case 2.2. Let $\Sigma_{S_{1}}$ be the subdiagram shown in Figure 11 b). By Corollary 1.2, we have $\Sigma_{S_{1}}=\bar{S}_{1}$. In particular, the nodes $a_{2}$ and $a_{3}$ cannot be neighbors of $S_{1}$, and therefore (by Corollary 1.2) they are joined with $y_{4}$. Furthermore, the diagram $\left\langle x_{1}, x_{2}, x_{3}, a_{3}\right\rangle$ is of type $F_{4}$ and has three neighbors, $x_{4}, a_{2}$, and $y_{4}$. Hence $a_{1}$ is not a neighbor of this diagram, and therefore $\left[a_{1}, x_{4}\right] \neq 2$ (since $a_{1}$ is a neighbor of $S_{0}=\left\langle x_{1}, x_{2}, x_{3}, x_{4}\right\rangle$ ). Consider the diagram $S_{6}=\left\langle a_{2}, x_{1}, x_{2}\right\rangle$ of type $H_{3}$. It has no good neighbors and $\bar{S}_{6}=\Sigma_{S_{6}}$. Furthermore, $\bar{S}_{6}$ contains the dashed edge $a_{3} x_{4}$, so $S_{6}$ has at most two bad neighbors. Then $S_{6}$ has exactly two neighbors, $x_{3}$ and $y_{4}$. Hence, $\left\langle a_{3}, x_{4}, a_{1}\right\rangle \subset \Sigma_{S_{6}}$, and $\Sigma_{S_{6}}$ is the diagram of a 4-prism. Thus, in the diagram of the 4-prism, the edge $\widetilde{x}_{4}, \widetilde{a}_{1}$ has a common node with a dashed edge, hence $\left[x_{4}, a_{1}\right]=3$. It follows that $\left\langle x_{1}, x_{2}, x_{3}, x_{4}, a_{1}\right\rangle$ is a parabolic diagram of type $\widetilde{F}_{4}$, which is impossible.

Case 2.3. Let $\Sigma_{S_{1}}$ be the diagram shown in Figure 11, c). Consider two cases: either $\left[a_{3}, x_{4}\right]=\infty$ or $\left[a_{3}, x_{4}\right] \neq \infty$ in $\Sigma$.

Case 2.3.1. Suppose that $\left[a_{3}, x_{4}\right]=\infty$. Since $a_{2}$ is not a good neighbor of $S_{1}, y_{4}$ is the only node of $\bar{S}_{0}$ joined with $a_{2}$. 
a)

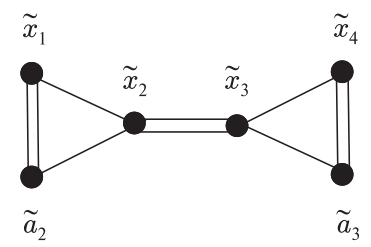

b)

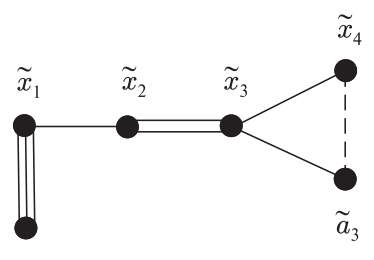

$\tilde{a}_{2}$

c)

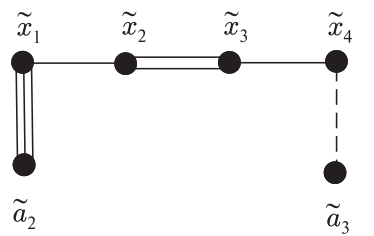

Figure 11. Diagrams of 4-polytopes with six facets containing $F_{4}$ and containing no $G_{2}^{(k)}$ with $k>5$

Consider the diagram $S_{7}=\left\langle a_{2}, x_{1}\right\rangle$ of type $G_{2}^{(5)}$. If $S_{7}$ has a bad neighbor, then $P\left(S_{7}\right)$ is a 5 -polytope with at most $5+3$ facets. Then $\Sigma_{S_{7}}$ contains the subdiagram $\left\langle\widetilde{x}_{2}, \widetilde{x}_{3}, \widetilde{x}_{4}, \widetilde{a}_{3}\right\rangle$, and $\widetilde{x}_{2} \widetilde{x}_{3}$ is a dashed edge in $\Sigma_{S_{7}}$. However, no diagram of a 5-polytope with at most $5+3$ facets contains two dashed edges $\left(\widetilde{x}_{2} \widetilde{x}_{3}\right.$ and $\left.\widetilde{x}_{4} \widetilde{a}_{3}\right)$, joined by a simple edge $\left(\widetilde{x}_{3} \widetilde{x}_{4}\right)$. Hence $S_{7}$ has no bad neighbors. In particular, $\left[a_{2}, y_{4}\right]=3$.

Consider the diagram $\left\langle a_{2}, S_{0}, \bar{S}_{0}\right\rangle$. It contains no dashed edges, and we know the multiplicities of all edges in this diagram except for the edges in the Lannér subdiagram $\bar{S}_{0}$. Since the number of Lannér diagrams of order 4 is finite, for $\left\langle a_{2}, S_{0}, \bar{S}_{0}\right\rangle$ there are only finitely many possibilities. None of these satisfies the signature condition. Therefore the case $\left[a_{3}, x_{4}\right]=\infty$ is impossible.

Case 2.3.2. Suppose that $\left[a_{3}, x_{4}\right] \neq \infty$. Then $a_{3}$ is a good neighbor of $S_{1}$ and therefore $\left[a_{3}, x_{4}\right]=5$ (otherwise either $\left[a_{3}, x_{4}\right]=3$ and $\left\langle a_{3}, S_{0}\right\rangle$ is a parabolic diagram of type $\widetilde{F}_{4}$ or $\left[a_{3}, x_{4}\right]=4$ and $\left\langle a_{3}, x_{4}, x_{3}, x_{2}\right\rangle$ is a parabolic diagram of type $\left.\widetilde{C}_{4}\right)$.

Consider the subdiagram $X=\left\langle S_{0}, \bar{S}_{0}, a_{3}\right\rangle$, which we know completely with the exception of finitely many possibilities for $\bar{S}_{0}\left(S_{0}\right.$ is of type $F_{4}, \bar{S}_{0}$ is a Lannér diagram of order $4, S_{1} \subset \bar{S}_{0}$ is of type $H_{3}$ or $B_{3}, a_{3}$ is a good neighbor of $S_{1}$, and $a_{3}$ is joined with $S_{0}$ as shown in Figure 11, $c$ )). The only diagram satisfying these conditions and the signature condition is

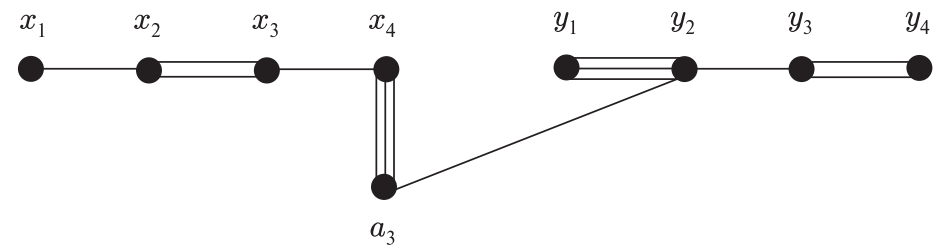

It is clear that $a_{2}$ is not a good neighbor of $S_{1}$, and therefore $a_{2}$ is not a neighbor of $S_{1}=\left\langle y_{1}, y_{2}, y_{3}\right\rangle$ and $\left[a_{2}, y_{4}\right] \neq 2$ (Lemma 1.4). Hence $S_{2}=\left\langle y_{2}, y_{3}, y_{4}\right\rangle$ has three bad 
neighbors: $a_{2}, a_{3}$, and $y_{1}$. Therefore $\Sigma_{S_{2}}$ is a Lannér diagram of order 5 containing a subdiagram of type $F_{4}$. It follows that $a_{1}$ is joined with $x_{1}$ and $x_{4}$, but then the diagram $\left\langle x_{4}, a_{3}, y_{3}, y_{2}\right\rangle$ of type $H_{4}$ has four bad neighbors $a_{1}, x_{3}, y_{1}, y_{4}$.

Thus, we have examined two prisms and an Esselmann polytope and in each case obtained a contradiction.

Case 3. Suppose that $S_{1}$ has three bad neighbors, $y_{4}, a_{1}$, and $a_{2}$. Then $\bar{S}_{1}$ is a cyclic Lannér diagram of order 5 (i.e., $\bar{S}_{1}=\mathcal{L}_{5}^{5}$ ), $\left[a_{3}, x_{1}\right]=\left[a_{3}, x_{4}\right]=3$, and $\left[a_{3}, x_{2}\right]=\left[a_{3}, x_{3}\right]=$ 2. By Corollary 1.2, $a_{3}$ is not a good neighbor of $S_{1}$, and therefore, $y_{4}$ is the only node joined with $a_{3}$.

Recall that $S_{2} \subset \bar{S}_{0}$ is a subdiagram of type $B_{3}$ or $H_{3}, S_{2}=\left\langle y_{2}, y_{3}, y_{4}\right\rangle$. As was shown in Cases 1 and 2, we may assume that $S_{2}$ also has three bad neighbors. Arguing as before (with $S_{2}$ in place of $S_{1}$ ), we have that the nodes $a_{i}, i=1,2,3$, cannot be good neighbors of $S_{2}$, and exactly one of these three nodes is not joined with $a_{i}$. Hence $a_{3}$ and one of the nodes $a_{1}$ and $a_{2}$ (say, $a_{1}$ ) are bad neighbors of $S_{2}$, and $a_{2}$ is not joined with this diagram. Moreover, $\left\langle a_{1}, S_{0}\right\rangle$ is a cyclic Lannér diagram and $y_{1}$ is the only node of $\bar{S}_{0}$ joined with $a_{1}$. Without loss of generality, we may assume that $a_{2}$ is joined with $\left\langle x_{1}, x_{2}, x_{3}\right\rangle$. Then the diagram $\left\langle a_{1}, x_{1}, x_{2}, x_{3}\right\rangle$ is of type $B_{4}$ and has three bad neighbors, $x_{1}, a_{2}$, and $a_{3}$. Therefore $y_{1}$ is a good neighbor of $\left\langle a_{1}, x_{1}, x_{2}, x_{3}\right\rangle$, i.e., $\left[y_{1}, a_{1}\right]=3$. Recall that $a_{1}$ is a bad neighbor of $S_{1}$. This means that either $\left[y_{1}, y_{2}\right]=4,5$ or $\left[y_{1}, y_{3}\right]=4,5$. Hence one of the nodes $y_{2}$ and $y_{3}$ is a bad neighbor of $\left\langle y_{1}, a_{1}, x_{1}, x_{2}, x_{3}\right\rangle$, which is impossible (since there are three other bad neighbors, $x_{1}, a_{2}$, and $a_{3}$ ).

Lemma 6.9. If $\Sigma$ contains a subdiagram of type $H_{4}$, then $\Sigma=\Sigma_{P_{7}}$.

Proof. Suppose that $S_{0}=\left\langle x_{1}, x_{2}, x_{3}, x_{4}\right\rangle$ is a subdiagram of $\Sigma$ of type $H_{4}$. By Lemmas 6.3 and 6.7 $S_{0}$ has exactly three neighbors; call them $a_{1}, a_{2}$, and $a_{3}$. Hence $P\left(S_{0}\right)$ is a 3 -simplex, and $\bar{S}_{0}=\Sigma_{S_{0}}=\left\langle y_{1}, y_{2}, y_{3}, y_{4}\right\rangle$ is a Lannér diagram of order 4 . Let $S_{1}=\left\langle y_{1}, y_{2}, y_{3}\right\rangle \subset \bar{S}_{0}$ be a subdiagram of type $H_{3}$ or $B_{3}$. Then $S_{1}$ has at least one bad neighbor, $y_{4}$. Consider the three cases where $S_{1}$ has one, two, or three bad neighbors, respectively.

Case 1. Suppose $S_{1}$ has only one bad neighbor, $y_{4}$. Then $P\left(S_{1}\right)$ is a 4-polytope with $4+3$ facets. Arguing as in Case 1 of Lemma 6.8 we see that for some $i \in\{1,2,3\}$ the diagram $\left\langle a_{i}, S_{0}, \bar{S}_{0}\right\rangle$, say $\left\langle a_{1}, S_{0}, \bar{S}_{0}\right\rangle$, has no dashed edges. By Lemma 6.4, there are finitely many possibilities for the diagram $\left\langle a_{1}, S_{0}, \bar{S}_{0}\right\rangle$. Recall that $S_{1} \subset \bar{S}_{0}$ is a subdiagram of type $H_{3}$ or $B_{3}$ with only one bad neighbor. There are only three possibilities for the diagram $\left\langle a_{1}, S_{0}, \bar{S}_{0}\right\rangle$ satisfying this condition and the signature condition. We list those diagrams in Figure 12 and consider them separately.

Consider the diagrams shown in Figure 12, $a$ ) and $b$ ). It is clear that $S_{1}=\left\langle y_{2}, y_{3}, y_{4}\right\rangle$. Suppose that $a_{2}$ is joined with $y_{3}$ or $y_{4}$. Since $a_{2}$ is not a bad neighbor of $S_{1}$, the diagram $\left\langle a_{2}, S_{1}\right\rangle$ is of type $F_{4}$, contrary to Lemma 6.8. Hence both $a_{2}$ and $a_{3}$ are joined with either $y_{1}$ or $y_{2}$ (Lemma 1.4), and therefore the subdiagram $\left\langle y_{1}, y_{2}, y_{3}\right\rangle$ is of type $H_{3}$ and has four bad neighbors $\left(y_{4}, a_{1}, a_{2}, a_{3}\right)$, which is impossible.

Consider the diagram shown in Figure 12, c). Without loss of generality, we may assume that $S_{1}=\left\langle y_{1}, y_{2}, y_{3}\right\rangle$. Each of the diagrams $S_{2}=\left\langle a_{1}, y_{1}, y_{2}, y_{3}\right\rangle$ and $S_{3}=$ $\left\langle a_{1}, y_{4}, y_{3}, y_{2}\right\rangle$ is of type $H_{4}$ and has two bad neighbors, $x_{4}$ and $y_{1}$ ( $y_{4}$ in the latter case). Hence each of these diagrams has at most one extra neighbor (Lemma 6.7). On the other hand, by Lemma 1.4, each of the nodes $a_{2}$ and $a_{3}$ is joined with $\left\langle a_{1}, y_{1}, y_{2}, y_{3}, y_{4}\right\rangle$. Hence we may assume that $a_{2}$ is not joined with $S_{2}$ and is joined with $y_{4}$, and $a_{3}$ is not joined with $S_{3}$ and is joined with $y_{1}$. Since $S_{1}$ has no other bad neighbors besides $y_{4}$, we have $\left[a_{4}, y_{1}\right]=3$. Furthermore, $\left[a_{3}, a_{1}\right]=2$ (otherwise the diagram $\left\langle S_{1}, a_{3}\right\rangle$ is of type $H_{4}$ with 
a)

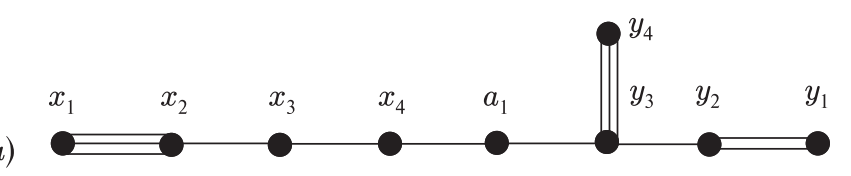

b)

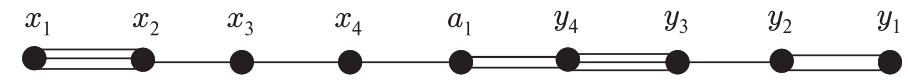

c)

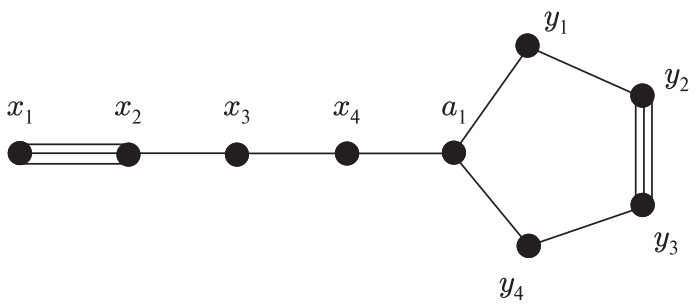

FIGURE 12. Three possibilities for $\left\langle a_{1}, S_{0}, \bar{S}_{0}\right\rangle$

four bad neighbors, $y_{4}, a_{2}, a_{1}$, and some $x_{i}, i \in\{1,2,3,4\}$, joined with $\left.a_{3}\right)$. If $\left[a_{1}, x_{4}\right]=2$, then $\left\langle x_{4}, a_{1}, y_{4}, y_{1}, y_{2}, a_{3}\right\rangle$ is a parabolic diagram of type $\widetilde{D}_{5}$, and therefore, $\left[a_{3}, x_{4}\right] \neq 2$. Moreover, $\left[a_{1}, x_{4}\right] \neq 3$ (otherwise $\left\langle x_{4}, a_{1}, y_{1}, a_{3}\right\rangle$ is a parabolic diagram of type $\widetilde{A}_{3}$ ). Hence $S_{4}=\left\langle x_{2}, x_{3}, x_{4}, a_{1}, y_{4}, y_{3}\right\rangle$ is of type $A_{6}$ and has three bad neighbors, $x_{1}, y_{2}$, and $a_{3}$. Thus, $a_{2}$ is a good neighbor of $S_{4},\left[a_{2}, y_{4}\right]=3$, and $\left[a_{2}, x_{4}\right]=\left[a_{2}, x_{3}\right]=\left[a_{2}, x_{2}\right]=2$. It follows that $\left\langle x_{3}, x_{4}, a_{1}, y_{4}, a_{2}, y_{1}, y_{2}\right\rangle$ is a parabolic diagram of type $\widetilde{E}_{6}$, which is impossible.

Case 2. Suppose that $S_{1}$ has two bad neighbors, $y_{4}$ and $a_{1}$. Then $P\left(S_{1}\right)$ is either an Esselmann polytope or a 4-prism, and

$$
\Sigma_{S_{1}}=\left\langle\widetilde{x}_{1}, \widetilde{x}_{2}, \widetilde{x}_{3}, \widetilde{x}_{4}, \widetilde{a}_{2}, \widetilde{a}_{3}\right\rangle .
$$

The Coxeter diagram of an Esselmann polytope not containing subdiagrams of type $F_{4}$ and $G_{2}^{(k)}, k \geq 6$, is one of the two diagrams shown in Figure 13. The Coxeter diagram of a 4-prism not containing subdiagrams of type $F_{4}$ or $G_{2}^{(k)}, k \geq 6$, is one of the diagrams shown in Figure 14. Diagram $a$ ) is shown three times, since there are three different ways of embedding $S_{0}$ in this diagram (in any other diagram containing more than one subdiagram of type $H_{4}$, those subdiagrams are permuted by automorphisms of the diagram).

We may assume that $\left\langle\widetilde{x}_{1}, \widetilde{x}_{2}, \widetilde{x}_{3}\right\rangle$ is a subdiagram of $\Sigma_{S_{1}}$ of type $H_{3}$. In any possible case for $\bar{S}_{1}$, that subdiagram has exactly one bad neighbor (in $\Sigma_{S_{1}}$ ). Assume that this is $\widetilde{a}_{2}$.

We first consider the Esselmann polytopes and then the prisms.

Case 2.1. Suppose that $\Sigma_{S_{1}}$ is the diagram of an Esselmann polytope (see Figure 13). The subdiagram $S_{5}=\left\langle x_{1}, x_{2}, x_{3}, a_{3}\right\rangle$ of type $H_{4}$ has three bad neighbors in $\Sigma\left(a_{2}, x_{4}\right.$, and some node of $\bar{S}_{0}$ joined with $\left.a_{3}\right)$. Therefore, $a_{1}$ is not a neighbor of $S_{5}=\left\langle x_{1}, x_{2}, x_{3}, a_{3}\right\rangle$, and $a_{1}$ is joined with $x_{4}$ (since $a_{1}$ is a neighbor of $S_{0}$ ). Furthermore, the diagram $\left\langle S_{0}, a_{3}, \bar{S}_{0}\right\rangle$ consists of the subdiagrams $\left\langle S_{0}, a_{3}\right\rangle$ and $\bar{S}_{0}$ joined by the edge $a_{3} y_{4}$ only (notice that this edge is not empty by Lemma 1.4). Moreover, this is a dashed edge; otherwise the diagram $\left\langle S_{0}, a_{3}, \bar{S}_{0}\right\rangle$ would be superhyperbolic. It follows that the three bad neighbors of $S_{5}=\left\langle x_{1}, x_{2}, x_{3}, a_{3}\right\rangle$ are $a_{2}, x_{4}, y_{4}$, and we conclude that $\left\langle S_{1}, a_{1}\right\rangle=\bar{S}_{5}=\Sigma_{S_{5}}$ is a Lannér diagram of order 4 . 
a)

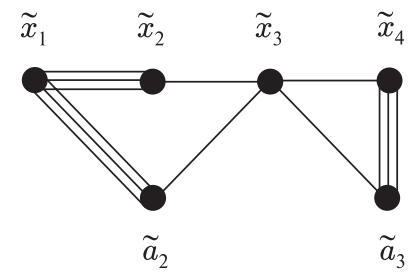

b)

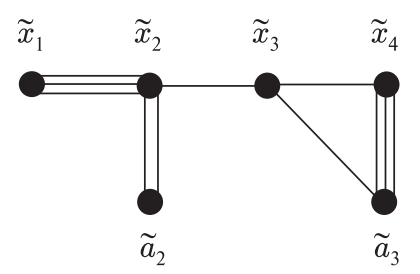

Figure 13. Diagrams of Esselmann polytopes containing $H_{4}$ and containing no $G_{2}^{(k)}$ with $k>5$

Consider the diagram $S_{6}=\left\langle a_{3}, x_{4}\right\rangle$ of type $G_{2}^{(5)}$. It has at least two bad neighbors, $y_{4}$ and $x_{4}$, and therefore $P\left(S_{6}\right)$ is a 5 -polytope with at most $5+2$ facets. Hence $P\left(S_{6}\right)$ is a 5 -prism. Notice that the diagram $X=\left\langle x_{1}, x_{2}, a_{2}, y_{1}, y_{2}, y_{3}\right\rangle$ is not joined with $S_{6}$ in $\Sigma$, so it does not differ from the subdiagram $\left\langle\widetilde{x}_{1}, \widetilde{x}_{2}, \widetilde{a}_{2}, \widetilde{y}_{1}, \widetilde{y}_{2}, \widetilde{y}_{3}\right\rangle$ in $\Sigma_{S_{6}}$. It is clear that $X$ contains no dashed edges $\left(a_{2}\right.$ cannot be joined with $S_{1}$ by a dashed edge, since $a_{2}$ is not a bad neighbor of $S_{1}$ ). Since the diagram of a 5-prism does not contain Lannér subdiagrams of order 3 , we have that $a_{2}$ is a good neighbor of $S_{1}, \Sigma_{S_{1}}$ is the diagram shown in Figure [13, b), $\left[a_{2}, x_{2}\right]=3$, and $S_{1}$ is a diagram of type $B_{3}$ (if $S_{1}$ was of type $H_{3}$, then $\widetilde{a}_{2} \widetilde{x}_{2}$ would be a dashed edge of $\Sigma_{S_{6}}$, and so it could not have a common node with the triple edge $\widetilde{x}_{1} \widetilde{x}_{2}$ in the diagram of a 5-prism). Without loss of generality, we may assume that $\left[y_{1}, y_{2}\right]=3$ and $\left[y_{2}, y_{3}\right]=4$. Thus, we have a linear diagram $\left\langle x_{1}, x_{2}, a_{2}, y_{1}, y_{2}, y_{3}\right\rangle$ with edges labeled by $5,3,3,3,4$. It follows that $\left[\widetilde{a}_{1}, \widetilde{y}_{3}\right]=\infty$ in $\Sigma_{S_{6}}$, and therefore $\left[a_{1}, y_{3}\right] \neq 2,3$ in $\Sigma$ (if $\left[a_{1}, y_{3}\right]=3$, then the edge $\widetilde{a}_{1} \widetilde{y}_{3}$ of $\Sigma_{S_{6}}$ would be labeled by 10 , but it should be a dashed edge). However, in the Lannér diagram $\left\langle S_{1}, a_{1}\right\rangle$ of order 4 , the multiple edge $a_{1} y_{3}$ cannot have a common node with the multiple edge $y_{3} y_{2}$.

The obtained contradiction shows that $\Sigma_{S_{1}}$ cannot be the diagram of an Esselmann polytope.

Case 2.2. Now suppose that $\Sigma_{S_{1}}$ is a 4-prism. Notice that in all diagrams of 4-prisms all edges incident to $a_{2}$ are simple, and by Corollary 1.2, $a_{2}$ cannot be a good neighbor of $S_{1}$. Hence $a_{2}$ is joined with $y_{4}$ (Lemma 1.4).

Furthermore, suppose that $\left[a_{2}, y_{4}\right] \neq \infty$. Then the diagram $\left\langle S_{0}, a_{2}, \bar{S}_{0}\right\rangle$ satisfies the following conditions: it has no dashed edges, $y_{4}$ is the only node of $\bar{S}_{0}$ joined with $a_{2}$, $a_{2}$ is a bad neighbor of $H_{3} \subset S_{0}$, and $a_{2}$ is joined with $S_{0}$ by simple edges only. None of the diagrams satisfies these conditions together with the signature condition, whence $\left[a_{2}, y_{4}\right]=\infty$.

We now examine the diagrams of 4-prisms case by case.

Case 2.2.1. Consider the diagram shown in Figure 14, $a 1$ ). If $a_{1}$ is joined with $\left\langle x_{1}, x_{2}, x_{4}\right\rangle$, then $\left\langle x_{2}, x_{1}, a_{2}, x_{4}\right\rangle$ is of type $H_{4}$ with four neighbors, $x_{3}, a_{3}, a_{1}, y_{4}$ (recall that $\left[a_{2}, y_{4}\right]=$ $\infty$ ). If $a_{1}$ is not a neighbor of $\left\langle x_{1}, x_{2}, x_{4}\right\rangle$, then $\left[a_{1}, x_{3}\right] \neq 2$ (as $a_{1}$ is a neighbor of $\left.S_{0}=\left\langle x_{1}, x_{2}, x_{3}, x_{4}\right\rangle\right)$ and $\left\langle x_{1}, x_{2}, x_{3}, a_{3}\right\rangle$ is of type $H_{4}$ with four neighbors $\left(x_{3}, a_{2}, a_{1}\right.$, and some node $y_{i}, i \in\{1,2,3,4\}$, joined with $a_{3}$; see Lemma 1.4). 
a1)

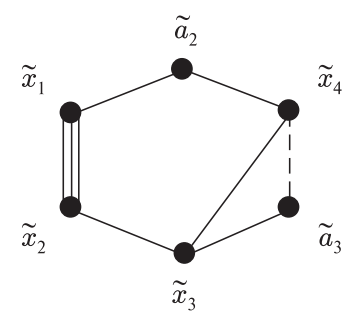

a2)

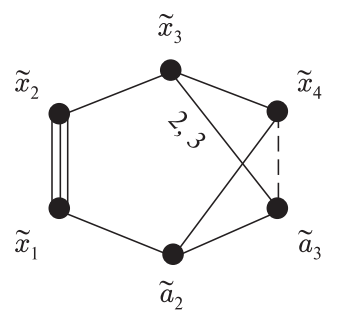

a3)

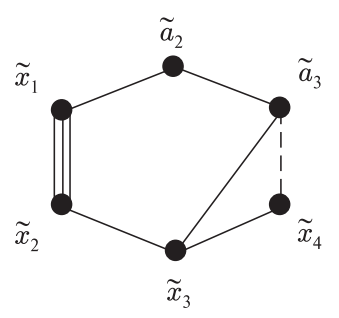

e)
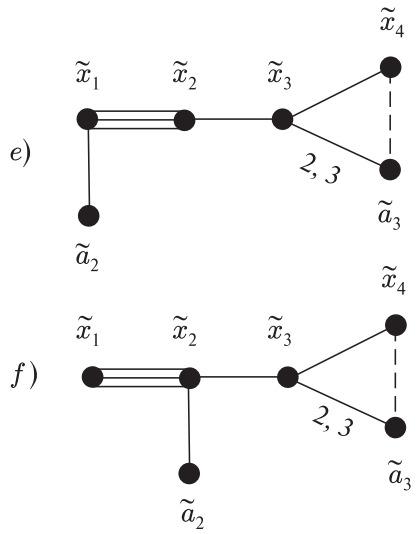

b)

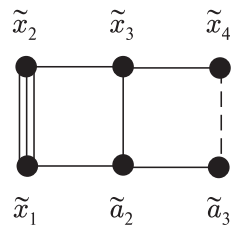

c)

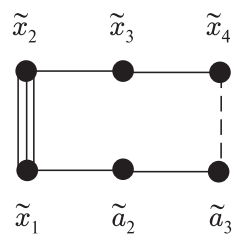

d)

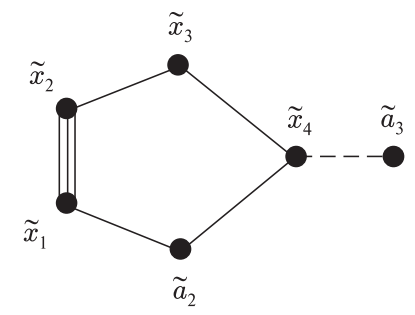

g)

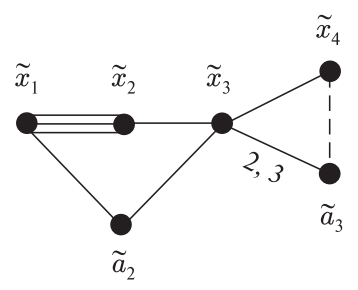

FiguRE 14. Diagrams of 4-prisms containing $H_{4}$

Case 2.2.2. Consider the diagrams shown in Figure 14, $a 2)-c$ ). Since $\left\langle x_{2}, x_{1}, a_{2}, a_{3}\right\rangle$ is of type $H_{4}$ and has three bad neighbors, $x_{3}, x_{4}$, and $y_{4}\left(\right.$ as $\left.\left[a_{2}, y_{4}\right]=\infty\right),\left\langle S_{1}, a_{1}\right\rangle$ is a Lannér diagram. It is clear that the nodes $x_{1}, x_{2}, a_{2}$ are not joined with that Lannér diagram (otherwise $\left\langle x_{2}, x_{1}, a_{2}, a_{3}\right\rangle$ would have four bad neighbors, $x_{3}, x_{4}, y_{4}$, and some node of $\left.\left\langle S_{1}, a_{1}\right\rangle\right)$. Since the Lannér diagrams $\left\langle a_{1}, S_{1}\right\rangle$ and $\left\langle x_{1}, x_{2}, x_{3}, a_{2}\right\rangle$ must be joined, we have $\left[x_{3}, a_{1}\right] \neq 2$. The signature condition, applied to the diagram $\left\langle S_{0}, a_{1}, a_{2}, S_{1}\right\rangle$, implies that $\left[x_{3}, a_{1}\right]=\infty$. This means that $\left\langle x_{2}, x_{3}, x_{4}, a_{2}\right\rangle$ is of type $A_{4}$ (in the case of $a 2)$ ), or $A_{3}+A_{1}$ (in the cases of $a 3$ ) and $c$ )), or $D_{4}$ (in the case of $b$ )) and has four bad neighbors, $x_{1}, a_{3}, y_{4}, a_{1}$ (here we again use the fact that $\left[a_{2}, y_{4}\right]=\infty$ ).

Case 2.2.3. Consider the diagram shown in Figure 14 $(d)$. Since the subdiagram $\left\langle x_{2}, x_{1}\right.$, $\left.a_{2}, x_{4}\right\rangle$ is of type $H_{4}$ and has three bad neighbors $\left(x_{3}, a_{3}\right.$ and $\left.y_{4}\right), a_{1}$ cannot be a neighbor 
of $\left\langle x_{2}, x_{1}, a_{2}, x_{4}\right\rangle$ and $\left\langle a_{1}, S_{1}\right\rangle$ is a Lannér diagram. Hence $\left[a_{1}, x_{3}\right] \neq 2\left(a_{1}\right.$ is a neighbor of $S_{0}=\left\langle x_{1}, x_{2}, x_{3}, x_{4}\right\rangle$, but not a neighbor of $\left.\left\langle x_{1}, x_{2}, x_{4}\right\rangle\right)$. The signature condition, applied to the diagram $\left\langle S_{0}, a_{1}, a_{2}, S_{1}\right\rangle$, implies that $\left[x_{3}, a_{1}\right]=\infty$.

Suppose $a_{3}$ is not a good neighbor of $S_{1}$. Then the dashed edge $\widetilde{x}_{4} \widetilde{a}_{3}$ of $\Sigma_{S_{1}}$ corresponds to the dashed edge $x_{4} a_{3}$ of $\bar{S}_{1}$, and the diagram $\left\langle x_{2}, x_{3}, x_{4}, a_{2}\right\rangle$ of type $A_{4}$ has four bad neighbors, $x_{1}, a_{1}, a_{3}, y_{4}$ (we used the facts that $\left[x_{3}, a_{1}\right]=\infty$ and $\left[a_{2}, y_{4}\right]=\infty$ ), which is impossible.

Suppose that $a_{3}$ is a good neighbor of $S_{1}$. If the edge $\left\langle x_{4}, a_{3}\right\rangle \subset \bar{S}_{1}$ is dashed (as is $\left\langle\widetilde{x}_{4}, \widetilde{a}_{3}\right\rangle \subset \Sigma_{S_{1}}$ ) or multiple, then $a_{3}$ is a bad neighbor of $\left\langle x_{2}, x_{3}, x_{4}, a_{2}\right\rangle$, and this diagram still has four bad neighbors. Hence we may assume that the edge $x_{4} a_{3}$ becomes simple in $\bar{S}_{1}$. This can only happen when $S_{1}$ is of type $H_{3}$ (otherwise, $\left\langle a_{2}, x_{3}, x_{4}, a_{3}, S_{1}\right\rangle$ is a parabolic diagram of type $\left.\widetilde{B}_{6}\right)$. Thus, we have the following diagram:

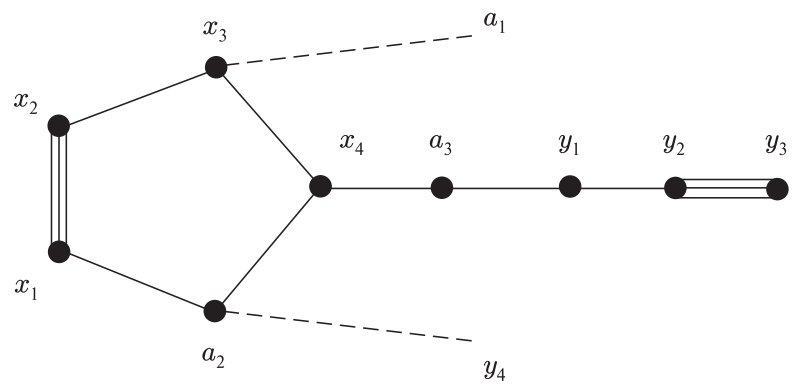

By Lemma 6.2. $\left[y_{4}, a_{3}\right] \neq \infty$. If $\left[y_{4}, a_{3}\right]=5$, then the diagram $\left\langle y_{4}, a_{3}, x_{4}, x_{3}\right\rangle$ is of type $H_{4}$ and has four bad neighbors, $a_{1}, a_{2}, y_{1}, x_{2}$. If $\left[y_{4}, a_{3}\right]=4$, then the diagram $\left\langle y_{4}, a_{3}, x_{4}, x_{3}, x_{2}\right\rangle$ is of type $B_{5}$ and has four bad neighbors, $a_{1}, a_{2}, y_{1}, x_{1}$. Hence $\left[y_{4}, a_{3}\right]=$ 2 or 3. Similarly, $\left[a_{1}, a_{3}\right]=2$ or 3 .

Consider now the diagram $\left\langle S_{0}, a_{3}, S_{1}\right\rangle$. The node $y_{4}$ is not joined with $S_{0}$. Since $\left\langle x_{2}, x_{3}, x_{4}, a_{3}, y_{1}, y_{2}\right\rangle$ has three bad neighbors $\left(x_{1}, a_{1}, y_{3}\right), y_{4}$ can be joined with $a_{3}$ by a simple edge only. Recall that $\left\langle S_{1}, y_{4}\right\rangle=\bar{S}_{0}$ is a Lannér diagram, so Lemma 1.4 implies that $\left[y_{4}, a_{3}\right] \neq 2$. Therefore, $\left[y_{4}, a_{3}\right]=3$. Examining the Lannér subdiagrams $\left\langle y_{4}, S_{1}\right\rangle$ (where $S_{1}$ is of type $H_{3}$ ), we find that the diagram $\left\langle S_{0}, a_{3}, y_{4}, S_{1}\right\rangle$ satisfies the signature condition only when $\left[y_{4}, y_{3}\right]=3$ and $\left[y_{4}, y_{1}\right]=\left[y_{4}, y_{2}\right]=2$. Similarly, examining the diagram $\left\langle x_{2}, x_{1}, a_{2}, x_{4}, a_{3}, a_{1}, S_{1}\right\rangle$, we have $\left[a_{1}, a_{3}\right]=\left[a_{1}, y_{3}\right]=3$ and $\left[a_{1}, y_{1}\right]=\left[a_{1}, y_{2}\right]=$ 2. However, in this case the diagram $\left\langle a_{3}, y_{4}, y_{3}, y_{2}\right\rangle$ is of type $H_{4}$ and has four neighbors $\left(a_{2}, x_{4}, y_{1}, a_{1}\right)$.

Case 2.2.4. Consider the diagrams shown in Figure 14, $e$ )-g). As before, $a_{2}$ cannot be a good neighbor of $S_{1}$, i.e., $a_{2}$ is not joined with $S_{1}$, and, as has been shown above, $\left[a_{2}, y_{4}\right]=\infty$. However, if $\left[x_{3}, a_{3}\right]=2$, then $a_{3}$ can be a good neighbor of $S_{1}$ and it may happen that $\left[x_{4}, a_{3}\right] \neq \infty$ in $\Sigma$.

Suppose that $\left[x_{4}, a_{3}\right] \neq \infty$ in $\Sigma$ and therefore $\left[x_{3}, a_{3}\right]=2$. Consider the diagram $Y=\left\langle S_{0}, a_{2}, a_{3}, S_{1}\right\rangle$. If $\left[x_{4}, a_{3}\right]=3$ or 5 , then the diagram $Y$ does not satisfy the signature condition (more precisely, at least one of its subdiagrams $Y \backslash a_{2}$ and $Y \backslash y_{4}$ does not satisfy the signature condition). If $\left[x_{4}, a_{3}\right]=4$, then $Y$ contains the parabolic diagram $\left\langle x_{2}, x_{3}, x_{4}, a_{3}, y_{1}\right\rangle$ of type $\widetilde{F}_{4}$ (here we assumed that the nodes of $S_{1}$ are numbered in such a way that $\left[y_{1}, y_{2}\right]=3$ and $\left[y_{2}, y_{3}\right]=4$ or 5$)$.

Thus, $\left[x_{4}, a_{3}\right]=\infty$. Consider the subdiagram $S_{7}=\left\langle x_{2}, x_{1}, a_{2}\right\rangle$ of type $H_{3}$. It has at least two bad neighbors, $x_{3}$ and $y_{4}$. Furthermore, the edge $x_{4} a_{3}$ is not joined with $S_{7}$, so $\left\langle x_{4}, a_{3}\right\rangle \subset \bar{S}_{7}$, and therefore $S_{7}$ has no bad neighbors besides $x_{3}$ and $y_{4}$. In particular, $a_{1}$ cannot be a bad neighbor of $S_{7}$. Suppose that $a_{1}$ is a good neighbor of $S_{7}$. Then $S_{8}=\left\langle a_{1}, S_{7}\right\rangle$ is of type $H_{4}$ with three bad neighbors $\left(x_{3}, y_{4}\right.$, and the node of $S_{1}$ joined 
with $a_{1}$ ). On the other hand, the diagram $\bar{S}_{8}=\Sigma_{S_{8}}$ contains the dashed edge $x_{4} a_{3}$, contrary to being a Lannér diagram of order 4 . The obtained contradiction shows that $a_{1}$ is not joined with $S_{7}$. Thus, $\bar{S}_{7}=\Sigma_{S_{7}}=\left\langle x_{4}, a_{3}, a_{1}, S_{1}\right\rangle$ is the diagram of a 4-prism, which implies that $\left\langle a_{1}, S_{1}\right\rangle$ is a Lannér diagram. It must be joined with the Lannér diagram $\left\langle a_{2}, x_{1}, x_{2}, x_{3}\right\rangle$. Therefore $\left[a_{1}, x_{3}\right] \neq 2$, since $a_{1}$ is not joined with $S_{7}$. Consider now the diagram $S_{9}=\left\langle a_{2}, x_{2}, x_{3}, x_{4}\right\rangle$ (of type $A_{1}+A_{3}, A_{4}$ or $D_{4}$ in the cases $e$ ), $f$ ), and $g$ ), respectively). The diagram $S_{9}$ has three bad neighbors, $x_{1}, a_{3}, y_{4}$, so $a_{1}$ is a good neighbor of $S_{9},\left[a_{1}, x_{3}\right]=3$, and $\left[a_{1}, x_{4}\right]=2$.

Finally, consider the diagram $Z=\left\langle S_{0}, a_{2}, a_{1}, S_{1}\right\rangle$. Since this diagram has no dashed edges, we have only finitely many possibilities for it. Moreover, $Z$ satisfies the following conditions: $\left\langle S_{0}, S_{1}\right\rangle$ is of type $H_{4}+H_{3}$ or $H_{4}+B_{3}, a_{2}$ is not joined with $S_{1}$ and is joined with $S_{0}$ in one of the ways shown in Figure 14, e) $\left.-g\right),\left[a_{1}, x_{3}\right]=3$, and $a_{1} x_{3}$ is the only edge joining $a_{1}$ with $\left\langle a_{2}, S_{0}\right\rangle ;\left\langle a_{1}, S_{1}\right\rangle$ is a Lannér diagram. However, no diagram satisfies all these conditions and the signature condition.

Case 3. Suppose that $S_{1}$ has three bad neighbors. Let $S_{1}^{\prime} \subset \bar{S}_{0}$ be a subdiagram of type $H_{3}$ or $B_{3}, S_{1}^{\prime} \neq S_{1}$ (see Lemma 1.5). Set $\left\langle y_{1}, y_{2}, y_{3}\right\rangle=S_{1}$ and $\left\langle y_{2}, y_{3}, y_{4}\right\rangle=S_{1}^{\prime}$. By Cases 1 and 2, we may assume that both $S_{1}$ and $S_{1}^{\prime}$ have three bad neighbors. There are two possibilities (up to a permutation of the nodes $a_{1}, a_{2}$, and $a_{3}$ ): either $a_{1}$ and $a_{2}$ are bad neighbors of both $S_{1}$ and $S_{1}^{\prime}$ (in addition to the bad neighbors $y_{4}$ and $y_{1}$ of $S_{1}$ and $S_{1}^{\prime}$, respectively) or $y_{4}, a_{1}, a_{2}$ are bad neighbors of $S_{1}$, and $y_{1}, a_{2}, a_{3}$ are bad neighbors of $S_{1}^{\prime}$.

Suppose that $a_{1}$ and $a_{2}$ are bad neighbors of each of $S_{1}$ and $S_{1}^{\prime}$. Then the node $a_{3}$ is not a bad neighbor of $S_{1}$ and $S_{1}^{\prime}$. Moreover, $\Sigma_{S_{1}}$ is a Lannér diagram of order 5 . By Corollary 1.2, $\bar{S}_{1}=\left\langle S_{0}, a_{3}\right\rangle$ is also a Lannér diagram. Thus, the diagram $\left\langle S_{0}, \bar{S}_{0}\right\rangle$ consists of the Lannér diagram $\left\langle S_{0}, a_{3}\right\rangle$ (where $S_{0}$ is a diagram of type $H_{4}$ ) and the Lannér diagram $\bar{S}_{0}$, the node $a_{3}$ being a bad neighbor of both $S_{1}$ and $S_{1}^{\prime}$. The only such diagram satisfying the signature condition is shown in Figure 12 b). But in this case, Proposition 1.11 implies that $\widetilde{x}_{4} \widetilde{a}_{3}$ is a dashed edge of $\Sigma_{S_{1}}$, contrary to the assumption that $\Sigma_{S_{1}}$ is a Lannér diagram of order 5 .

Thus we may assume that $y_{4}, a_{1}, a_{2}$ are bad neighbors of $S_{1}$, and $y_{1}, a_{2}, a_{3}$ are bad neighbors of $S_{1}^{\prime}$. Examining $\bar{S}_{1}$ and $\bar{S}_{1}^{\prime}$, we conclude that $\left\langle S_{0}, a_{1}\right\rangle$ and $\left\langle S_{0}, a_{3}\right\rangle$ are Lannér diagrams.

Consider now two cases: either both $a_{1}$ and $a_{3}$ are joined with $\bar{S}_{0}$ by dashed edges or at least one of the nodes $a_{1}$ and $a_{3}$ (say, $a_{1}$ ) is joined with $\bar{S}_{0}$ by ordinary edges only.

Case 3.1. Suppose that $a_{1}$ and $a_{3}$ are joined with $\bar{S}_{0}$ by dashed edges. Since $a_{1}$ is a bad neighbor of $S_{1}$ but not a bad neighbor of $S_{1}^{\prime}$, the dashed edge joining $a_{1}$ with $\bar{S}_{0}$ can only be $a_{1} y_{1}$. Similarly, $\left[a_{3}, y_{4}\right]=\infty$. Lemma 6.2 implies that $\left\langle a_{1}, a_{3}\right\rangle \neq \infty$. Hence we have only finitely many possibilities for the diagram $\left\langle S_{0}, a_{1}, a_{3}\right\rangle$ (there are four possibilities for each of the Lannér diagrams $\left\langle S_{0}, a_{1}\right\rangle$ and $\left\langle S_{0}, a_{3}\right\rangle$ and three possibilities for $\left.\left[a_{1}, a_{3}\right] \in\{3,4,5\}\right)$.

Consider the diagram $S_{10}=\left\langle x_{2}, x_{3}, x_{4}, a_{1}\right\rangle$ of type $D_{4}, A_{4}, B_{4}$, or $H_{4}$. If $a_{3}$ is a bad neighbor of $S_{10}$, then $S_{10}$ has three bad neighbors, $x_{1}, y_{1}$, and $a_{3}$, and therefore $a_{2}$ cannot be a bad neighbor of it. If $a_{3}$ is not a bad neighbor of $S_{10}$, then $\left\langle S_{10}, a_{3}\right\rangle$ has three bad neighbors $x_{1}, y_{1}, y_{4}$, and $a_{2}$ cannot be a bad neighbor of $\left\langle S_{10}, a_{3}\right\rangle$. In any case, $a_{2}$ cannot be a bad neighbor of $S_{10}$. Similarly, $a_{2}$ cannot be a bad neighbor of $S_{11}=\left\langle x_{2}, x_{3}, x_{4}, a_{3}\right\rangle$.

Suppose that $a_{2}$ is a bad neighbor of the diagram $S_{12}=\left\langle x_{1}, x_{2}, x_{3}\right\rangle$ of type $H_{3}$. Then $P\left(S_{12}\right)$ is a 4-polytope with at most $4+3$ facets. However, $\Sigma_{S_{12}} \neq \bar{S}_{12}$ and $\Sigma_{S_{12}}$ contains four dashed edges $\widetilde{y}_{4} \widetilde{a}_{3}, \widetilde{a}_{3} \widetilde{x}_{4}, \widetilde{x}_{4} \widetilde{a}_{1}, \widetilde{a}_{1} \widetilde{y}_{1}$, which is impossible for a 4-polytope with at most seven facets. Hence $a_{2}$ cannot be a bad neighbor of $S_{12}$, which implies that $a_{2}$ is 
not a neighbor of $x_{1} x_{2}$. Therefore, $a_{2}$ is a neighbor of $x_{3} x_{4}$ and each of the diagrams $S_{10}$ and $S_{11}$ is of type $D_{4}$ or $A_{4}$.

Suppose that $S_{10}$ and $S_{11}$ are of the same type. Then $\left[a_{1}, a_{3}\right]=2$ (otherwise either $\left[a_{1}, a_{3}\right]=\infty$, contrary to Lemma 6.2 , or the edge $a_{1} a_{3}$ has three bad neighbors, contrary to Lemma 6.1, or one of the diagrams $\left\langle x_{3}, a_{1}, a_{3}\right\rangle$ and $\left\langle x_{4}, a_{1}, a_{3}\right\rangle$ is parabolic of type $\left.\widetilde{A}_{2}\right)$. In this case $\left\langle S_{10}, a_{3}, a_{2}\right\rangle$ contains a parabolic subdiagram. Hence $S_{10}$ and $S_{11}$ are of different types. If $\left[a_{1}, a_{3}\right]=2$ or 3 , then $\left\langle S_{10}, a_{3}, a_{2}\right\rangle$ still contains a parabolic subdiagram. If $\left[a_{1}, a_{3}\right]=4$ or 5 , then $S_{13}=\left\langle a_{1}, a_{3}\right\rangle$ has two bad neighbors, so $\Sigma_{S_{13}}$ is the diagram of a 5-prism. However, in $\Sigma_{S_{13}}$ the subdiagram $\left\langle\widetilde{x}_{1}, \widetilde{x}_{2}, \widetilde{x}_{3}, \widetilde{x}_{4}\right\rangle$ is a linear diagram in which a triple edge and a simple edge are joined by the dashed edge $\widetilde{x}_{2} \widetilde{x}_{3}$. No diagram of a 5-prism contains such a subdiagram, which shows that Case 3.1 is impossible.

Case 3.2. Suppose that $a_{1}$ is joined with $S_{0}$ by ordinary (i.e., non-dashed) edges. Then the subdiagram $T=\left\langle S_{0}, a_{1}, S_{0}\right\rangle$ contains no dashed edges, and we have finitely many possibilities for the diagram $T$. Notice that $T$ satisfies the following conditions:

$S_{0}$ is of type $H_{4}$, and $\left\langle S_{0}, a_{1}\right\rangle$ is a Lannér diagram;

$\bar{S}_{0}$ is a Lannér diagram of order 4 , and $\bar{S}_{0}$ is not joined with $S_{0}$;

$S_{1}$ and $S_{1}^{\prime}$ are subdiagrams of $\bar{S}_{0}$ of type $H_{3}$ or $B_{3}$;

$a_{1}$ is a bad neighbor of $S_{1}$ not joined with $S_{1}^{\prime}$.

However, only two subdiagrams satisfy these conditions together with the signature condition; they are shown in Figure 15, $a$ ) and $b$ ).

Consider the diagram shown in Figure $15(a)$. The subdiagram $\left\langle x_{2}, x_{3}, x_{4}, a_{1}, y_{1}\right\rangle \subset \Sigma$ is of type $B_{5}$ and has three bad neighbors, $x_{1}, y_{2}$, and $a_{3}$. Hence $a_{2}$ cannot be a bad neighbor of $\left\langle x_{2}, x_{3}, x_{4}, a_{1}, y_{4}\right\rangle$, and so $a_{2}$ is joined with $x_{1} x_{2}$. Therefore $a_{2}$ is a bad neighbor of $S_{14}=\left\langle\widetilde{x}_{1}, \widetilde{x}_{2}, \widetilde{x}_{3}\right\rangle$, and $P\left(S_{14}\right)$ is a 4-polytope with $4+3$ facets (it is not difficult to check that $S_{14}$ has no other bad neighbors). However, $\Sigma_{S_{14}}$ contains the subdiagram $\left\langle\widetilde{a}_{1}, \widetilde{y}_{1}, \widetilde{y}_{2}\right\rangle$ consisting of the double edge $\widetilde{a}_{1} \widetilde{y}_{1}$ adjacent to the triple edge $\widetilde{y}_{1} \widetilde{y}_{2}$. But no diagram of a 4-polytope with $4+3$ facets has such a subdiagram, so the case shown in Figure 15, a) is impossible.

In particular, we conclude that for any diagram $S \subset \Sigma$ of type $H_{4}$, the diagram $Q=\left\langle S, \bar{S}, a_{1}\right\rangle$ is the diagram shown in Figure 15, $\left.b\right)$, and $\bar{S}$ is a linear Lannér diagram with one double, one simple, and one triple edge.

Consider the diagram $\left\langle Q, a_{3}\right\rangle$. By Lemma 6.2 $\left\langle Q, a_{3}\right\rangle$ contains at most one dashed edge (since any such edge must be incident to $a_{3}$ and cannot have endpoints in either $S_{0}$ (since $\left\langle S_{0}, a_{3}\right\rangle$ is a Lannér diagram) or $S_{1}$ (since $a_{3}$ is not a bad neighbor of $S_{1}$ ). Hence, either $\left\langle S_{0}, a_{3}, y_{4}, S_{1}\right\rangle$ or $\left\langle S_{0}, a_{3}, a_{1}, S_{1}\right\rangle$ contains no dashed edges. The former diagram does not satisfy the signature condition, whereas the latter satisfies it only in the case of the diagram shown in Figure 15 $c$ ). Moreover, $\left[a_{3}, y_{4}\right]=\infty$, since otherwise $\operatorname{det}\left(\left\langle S_{0}, a_{1}, a_{3}, \bar{S}_{0}\right\rangle\right) \neq 0$.

It remains to determine in how many ways the remaining node $a_{2}$ can be adjoined to the diagram $\left\langle S_{0}, a_{1}, a_{3}, \bar{S}_{0}\right\rangle$. Consider the diagram $S_{15}=\left\langle\widetilde{y}_{1}, \widetilde{y}_{2}, \widetilde{a}_{1}, \widetilde{x}_{4}\right\rangle$ of type $H_{4}$ with bad neighbors $x_{3}, a_{3}, y_{2}$. As has been shown before, $\bar{S}_{15}=\left\langle x_{1}, x_{2}, a_{2}, y_{4}\right\rangle$ is a linear Lannér diagram with one double, one simple, and one triple edge, i.e., either $\left[a_{2}, x_{2}\right]=$ $2,\left[a_{2}, x_{1}\right]=3,\left[a_{2}, y_{4}\right]=4$ or $\left[a_{2}, x_{2}\right]=3,\left[a_{2}, x_{1}\right]=2,\left[a_{2}, y_{4}\right]=4$. Furthermore, $a_{2}$ is not joined with $\left\langle x_{3}, x_{4}, a_{3}, a_{1}\right\rangle$ since the diagram $\left\langle x_{2}, x_{3}, x_{4}, a_{3}, a_{1}, y_{2}\right\rangle$ of type $E_{6}$ already has three bad neighbors, $x_{1}, y_{1}$, and $y_{4}$. Also, $a_{2}$ is not joined with the edge $y_{1} y_{2}$, since the diagram $\left\langle y_{1}, y_{2}, a_{1}, x_{4}\right\rangle$ of type $H_{4}$ already has three bad neighbors, $x_{3}, a_{3}$, and $y_{3}$. Since $a_{2}$ is a bad neighbor of $S_{1}$, we have $\left[a_{2}, y_{3}\right] \neq 2$. If $\left[a_{2}, y_{3}\right]=3$, then $S_{16}=\left\langle x_{2}, x_{1}, a_{2}, y_{3}\right\rangle$ is a diagram of type $H_{4}$ such that $\bar{S}_{16}=\left\langle y_{1}, a_{1}, x_{4}, a_{3}\right\rangle$ is a nonconnected diagram, which is impossible. If $\left[a_{2}, y_{3}\right]=5$ (or 4 ), then $\left\langle a_{2}, y_{3}, y_{2}, a_{1}\right\rangle$ (or 
a)
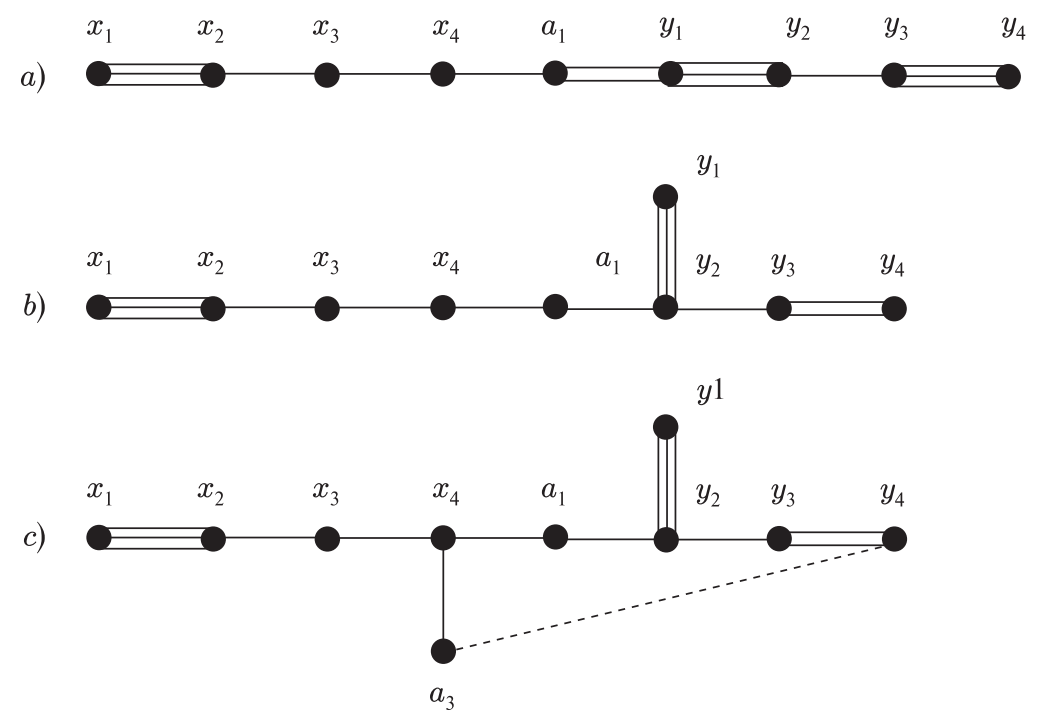

FiguRE 15. To the proof of Lemma 6.9

$\left\langle a_{2}, y_{3}, y_{2}, a_{1}, x_{4}, a_{3}\right\rangle$, respectively) is a diagram of type $H_{4}$ (or $B_{6}$, respectively) with four bad neighbors, $y_{4}, y_{1}, x_{4}$, and one of the nodes $x_{1}$ and $x_{2}$. Hence $\left[a_{2}, y_{3}\right]=\infty$.

Thus, $a_{2}$ is incident to three edges: the dashed edge $a_{2} y_{3}$, the double edge $a_{2} y_{4}$, and the simple edge $a_{2} x_{1}$ or $a_{2} x_{2}$. If $\left[a_{2}, x_{1}\right]=3$, then the diagram $\left\langle S_{0}, a_{1}, a_{2}, a_{3}, y_{1}, y_{2}\right\rangle$ does not satisfy the signature condition. Then $\left[a_{2}, x_{1}\right]=2,\left[a_{2}, x_{2}\right]=3$, and we have the diagram $\Sigma_{P_{7}}$.

Thus, having exhausted all the cases, we found only the polytope $P_{7}$, as claimed.

\section{REFERENCES}

[1] D. Allcock, Infinitely many hyperbolic Coxeter groups through dimension 19, Geom. Topol. 10 (2006), 737-758. MR2240904 (2007f:20067)

[2] E. M. Andreev, The intersection of the planes of the faces of polyhedra with acute angles, Mat. Zametki 8 (1970), 521-527; English transl., Math. Notes 8 (1970), 761-764. MR0279680 (43:5401)

[3] E. M. Andreev, Convex polyhedra in Lobachevsky spaces, Mat. Sb. (N.S.) 81 (1970), 445-478. (Russian) MR0259734 (41:4367)

[4] R. Borcherds, Coxeter groups, Lorentzian lattices, and K3 surfaces, IMRN 19 (1998), 1011-1031. MR1654763(2000a:20088)

[5] V. O. Bugaenko, Groups of automorphisms of unimodular hyperbolic quadratic forms over the ring $\mathbf{Z}[(\sqrt{5}+1) / 2]$, Vestnik Moskov. Univ. Ser. I Mat. Mekh. 5 (1984), 6-12. MR764026 (86d:11030)

[6] V. O. Bugaenko, Arithmetic crystallographic groups generated by reflections, and reflective hyperbolic lattices, Adv. Sov. Math. 8 (1992), 33-55. MR.1155663 (93g:20094)

[7] V. A. Emelichev, M. M. Kovalev, and M. K. Kravtsov, Polytopes, graphs and optimization, Nauka, Moscow, 1981; English transl., Cambridge University Press, Cambridge, 1984. MR0656517 (83h:52014) MR0744197 (85b:52008)

[8] F. Esselmann, Uber kompakte hyperbolische Coxeter-Polytope mit wenigen Facetten, Universität Bielefeld, SFB 343, Preprint No. 94-087.

[9] F. Esselmann, The classification of compact hyperbolic Coxeter d-polytopes with $d+2$ facets, Comment. Math. Helvetici 71 (1996), 229-242. MR1396674 (97j:52015)

[10] A. A. Felikson and P. V. Tumarkin, On subgroups generated by reflections in groups generated by reflections, Funct. Anal. Appl. 38 (2004), 313-314. MR2117513 (2005j:20046)

[11] A. A. Felikson and P. V. Tumarkin, On hyperbolic Coxeter polytopes with mutually intersecting facets, J. Combin. Theory Ser. A 115 (2008), 121-146, arXiv:math.MG/0604248. MR2378860 (2008m:52026)

[12] B. Grünbaum, Convex polytopes, John Wiley \& Sons, New York, 1967. MR0226496 (37:2085) 
[13] I. M. Kaplinskaya, The discrete groups that are generated by reflections in the faces of simplicial prisms in Lobachevsky spaces, Mat. Zametki 15 (1974), 159-164. (Russian) MR0360858 (50:13305)

[14] F. Lannér, On complexes with transitive groups of automorphisms, Comm. Sem. Math. Univ. Lund 11 (1950), 1-71. MR0042129(13:58c)

[15] H. Poincaré, Théorie des groups fuchsiennes, Acta Math. 1 (1882), 1-62. MR 1554574

[16] M. N. Prokhorov, Absence of discrete groups of reflections with a noncompact fundamental polyhedron of finite volume in a Lobachevsky space of high dimension, Izv. Akad. Nauk SSSR Ser. Mat. 50 (1986), 413-424. MR842588 (87k:22016)

[17] P. Tumarkin, Compact hyperbolic Coxeter n-polytopes with $n+3$ facets, Electron. J. Combin. 14 (2007), no. 1, Research Paper 69, 36 pp. MR2350459 (2008k:52022)

[18] È. B. Vinberg, Absence of crystallographic groups of reflections in Lobachevskiu spaces of large dimension, Trudy Moskov. Mat. Obshch. 47 (1984), 68-102. (Russian) MR774946 (86i:22020)

[19] È. B. Vinberg, Hyperbolic groups of reflections, Russian Math. Surveys 40 (1985), 31-75. MR783604 (86m:53059)

[20] Ė. B. Vinberg, O. V. Shvartsman, Discrete groups of motions of spaces of constant curvature, Geometry, II, Encyclopaedia Math. Sci., vol. 29, pp. 139-248, Springer, Berlin, 1993. MR1254933 (95b:53043)

IndePENDENT University OF Moscow, Russia

E-mail address: pasha@mccme.ru

IndePEndent University of Moscow, Russia

E-mail address: felikson@mccme.ru 